\title{
Calculating the Higgs mass in string theory
}

\author{
Steven Abel ${ }^{1, *}$ and Keith R. Dienes $\odot^{2,3, \dagger}$ \\ ${ }^{1}$ IPPP, Durham University, Durham DH1 3LE, United Kingdom \\ ${ }^{2}$ Department of Physics, University of Arizona, Tucson, Arizona 85721, USA \\ ${ }^{3}$ Department of Physics, University of Maryland, College Park, Maryland 20742, USA
}

(Received 22 June 2021; accepted 2 December 2021; published 29 December 2021)

\begin{abstract}
In this paper, we establish a fully string-theoretic framework for calculating one-loop Higgs masses directly from first principles in perturbative closed-string theories. Our framework makes no assumptions other than world sheet modular invariance and is therefore applicable to all closed strings, regardless of the specific string construction utilized. This framework can also be employed even when spacetime supersymmetry is broken (and even when this breaking occurs at the Planck scale), and can be utilized for all scalar Higgs fields, regardless of the particular gauge symmetries they break. This therefore includes the Higgs field responsible for electroweak symmetry breaking in the Standard Model. Notably, using our framework, we demonstrate that a gravitational modular anomaly generically relates the Higgs mass to the one-loop cosmological constant, thereby yielding a string-theoretic connection between the two fundamental quantities which are known to suffer from hierarchy problems in the absence of spacetime supersymmetry. We also discuss a number of crucial issues involving the use and interpretation of regulators in UV/IR-mixed theories such as string theory, and the manner in which one can extract an effective field theory (EFT) description from such theories. Finally, we analyze the running of the Higgs mass within such an EFT description, and uncover the existence of a "dual IR" region which emerges at high energies as the consequence of an intriguing scaleinversion duality symmetry. We also identify a generic stringy effective potential for the Higgs fields in such theories. Our results can therefore serve as the launching point for a rigorous investigation of gauge hierarchy problems in string theory.
\end{abstract}

DOI: $10.1103 /$ PhysRevD.104.126032

\section{INTRODUCTION}

Extracting phenomenological predictions from string theory is a subtle task. Chief among the complications is the question of finding a suitable vacuum. Without solving this problem, one is limited to making generic statements that might hold across broad classes of string theories. But even within the context of specific string models with certain favorable characteristics, most attempts at extracting the corresponding phenomenological predictions follow a common path. First, one tallies the massless states that arise in such models. Then, one constructs a field-theoretic Lagrangian which describes the dynamics of these states. Finally, one proceeds to analyze this Lagrangian using all of the regular tools of quantum field theory without further regard for the origins of these states within string theory.

\footnotetext{
s.a.abel@durham.ac.uk

†dienes@arizona.edu
}

Published by the American Physical Society under the terms of the Creative Commons Attribution 4.0 International license. Further distribution of this work must maintain attribution to the author(s) and the published article's title, journal citation, and DOI. Funded by SCOAP.
Although such a treatment may be sufficient for certain purposes, calculations performed in this manner have a serious shortcoming: by disregarding the infinite towers of string states that necessarily accompany these low-lying modes within the full string theory, such calculations implicitly disregard many of the underlying string symmetries that ultimately endow string theory with a plethora of remarkable properties that transcend our field-theoretic expectations. At first glance, it may seem that these extra towers of states cannot play an important role for lowenergy physics because these states typically have masses which are set by the string scale (generically assumed near the Planck scale) or by the scales associated with the compactification geometry. For this reason it would seem that these heavy states can legitimately be integrated out of the theory, thereby justifying a treatment based on a Lagrangian description of the low-lying modes alone, along with possible higher-order operators suppressed by powers of these heavier scales. However, it is difficult to justify integrating out infinite towers of states, much less towers whose state degeneracies at each mass level grow exponentially with mass. Yet this is precisely the situation we face in string theory. Indeed, these infinite towers of states particularly affect those operators (such as those 
associated with the Higgs mass and the cosmological constant) which have positive dimension and are therefore sensitive to all mass scales in the theory.

Many of the string symmetries that rely on these infinite towers of states go beyond what can be incorporated within the framework of an effective field theory (EFT). For example, strong/weak coupling duality relations intrinsically rely on the presence of the full towers of string states, both perturbative and nonperturbative. But there also exist stringy symmetries that operate purely within the perturbative weak-coupling regime. A prime example of this is T-duality, under which the physics of closed strings compactified on small compactification volumes is indistinguishable from the physics associated with strings compactified on large compactification volumes. This sort of equivalence between ultraviolet (UV) and infrared (IR) physics cannot be incorporated within an EFT-based approach in which we integrate out heavy states while treating light states as dynamical.

Both strong/weak coupling duality and T-duality are spacetime symmetries. As such, like all spacetime physics, they are merely the consequences of an underlying string theory. But closed-string theories have another symmetry of this sort which is even more fundamental and which must be imposed for consistency directly on the world sheet. This is world sheet modular invariance, which will be the focus of this paper. World sheet modular invariance is crucial since it lies at the heart of many of the finiteness properties for which string theory is famous. Moreover, since modular invariance is an exact symmetry of all perturbative closed-string vacua, it provides tight constraints on the spectrum of string states at all mass scales as well as on their interactions. Indeed, this symmetry is the ultimate "UV-IR mixer," operating over all scales and enforcing a delicate balancing between low-scale and high-scale physics. There is no sense in which its breaking can be confined to low energies, and likewise there is no sense in which it can be broken by a small amount. As an exact symmetry governing string dynamics, world sheet modular invariance is preserved even as the theory passes through phase transitions such as the Standard Model electroweak or QCD phase transitions, as might occur under cosmological evolution. Indeed, any shifts in the low-energy degrees of freedom induced by such phase transitions are automatically accompanied by corresponding shifts in the high-scale theory such that modular invariance is maintained and finiteness is preserved. Yet this entire structure is missed if we integrate out the heavy states and concentrate on the light states alone.

While certain phenomenological questions are not likely to depend on such symmetries, this need not always be the case. For example, these symmetries are likely to be critical for addressing fundamental questions connected with finiteness and/or the stability of (or even the coexistence of) different scales under radiative corrections.
Chief among these questions are hierarchy problems, which provide clues as to the UV theory and its potential connections to IR physics. Indeed, two of the most pressing mysteries in physics are the hierarchy problems associated with the cosmological constant and with the masses of scalar fields such as the Higgs field. However, integrating out the heavy string states eliminates all of the stringy physics that may provide alternative ways of addressing such problems. The lesson, then, is clear: If we are to take string theory literally as a theory of physics, then we should perform our calculations within the full framework of string theory, incorporating all of the relevant symmetries and infinite towers of states that string theory provides.

With this goal in mind, we begin this paper by establishing a fully string-theoretic framework for calculating one-loop Higgs masses directly from first principles in perturbative closed-string theories. This is the subject of Sec. II. Our framework will make no assumptions other than world sheet modular invariance and will therefore be applicable to all closed strings, regardless of the specific string construction utilized. Our results will thus have a generality that extends beyond individual string models. As we shall see, this framework operates independently of spacetime supersymmetry, and can be employed even when spacetime supersymmetry is broken (or even when the string model has no spacetime supersymmetry to begin with at any scale). Likewise, our framework can be utilized for all scalar Higgs fields, regardless of the particular gauge symmetries they break. This therefore includes the Higgs field responsible for electroweak symmetry breaking in the Standard Model.

One of the central results emerging from our framework is a relationship between the Higgs mass and the one-loop cosmological constant. This connection arises as the result of a gravitational modular anomaly, and is thus generic for all closed-string theories. This then provides a string-theoretic connection between the two fundamental quantities which are known to suffer from hierarchy problems in the absence of spacetime supersymmetry. From the perspective of ordinary quantum field theory, such a relation between the Higgs mass and the cosmological constant would be entirely unexpected. Indeed, quantum field theories are insensitive to the zero of energy. String theory, by contrast, unifies gauge theories with gravity. Thus, it is only within a string context that such a relation could ever arise. As we shall see, this relationship does not require supersymmetry in any form. It holds to one-loop order, but its direct emergence as the result of a fundamental string symmetry leads us to believe that it actually extends more generally. We stress that it is not the purpose of this paper to actually solve either of these hierarchy problems (although we shall return to this issue briefly in Sec. VI). However, we now see that these two hierarchies are connected in a deep way within a string context.

As we shall find, the Higgs mass receives contributions from all of the states throughout the string spectrum which 
couple to the Higgs in specified ways. This includes the physical (level-matched) string states as well as the unphysical (non-level-matched) string states. Depending on the string model in question, we shall also find that our expression for the total Higgs mass can be divergent; ultimately this will depend on the charges carried by the massless states. Accordingly, we shall then proceed to develop a set of regulators which can tame the Higgs-mass divergences while at the same time allowing us to express the Higgs mass as a weighted supertrace over only the physical string states. Developing these regulators is the subject of Sec. III. To do this, we shall begin by reviewing prior results in the mathematics literature which will form the basis for our work. Building on these results, we will then proceed to develop a set of regulators which are completely general, which preserve modular invariance, and which can be used in a wide variety of contexts even beyond their role in regulating the Higgs mass.

In Sec. IV, we shall then use these modular-invariant regulators in order to recast our results for the Higgs mass in a form that is closer to what we might expect in field theory. This will also allow us to develop an understanding of how the Higgs mass "runs" in string theory and to develop a physical "renormalization" prescription that can operate at all scales. Toward this end, we begin in Sec. IV A with a general discussion of how (and to what extent) one can meaningfully extract an effective field theory from UV/IR-mixed theories such as modular-invariant string theories. This issue is surprisingly subtle, since modular invariance relates UV and IR divergences to each other while at the same time softening both. For example, we shall demonstrate that while the Higgs mass is quadratically divergent in field theory, modular invariance renders the Higgs mass at most logarithmically divergent in string theory. We shall then apply our regulators from Sec. III to our Higgs-mass results in Sec. II and thereby demonstrate how the Higgs mass "runs" as a function of an energy scale $\mu$. The results of our analysis are highlighted in Fig. 3, which not only exhibits features which might be expected in an ordinary effective field theory but also includes features which clearly transcend traditional quantum field-theoretic expectations. The latter include the existence of a "dual" infrared region at high energy scales as well as an invariance under an intriguing "scale-duality" transformation $\mu \rightarrow M_{s}^{2} / \mu$, where $M_{s}$ denotes the string scale. This scale-inversion duality symmetry in turn implies the existence of a fundamental limit on the extent to which a modular-invariant theory such as string theory can exhibit UV-like behavior.

All of our results in Secs. II-IV are formulated in a fashion that assumes that our modular-invariant string theories can be described through charge lattices. However, it turns out that our results can be recast in a completely general fashion that does not require the existence of a charge lattice. This is the subject of Sec. V. Moreover, we shall find that this reformulation has an added benefit, allowing us to extract a modular-invariant stringy effective potential for the Higgs from which the Higgs mass can be obtained through a modular-covariant double derivative with respect to fluctuations of the Higgs field. This potential therefore sits at the core of our string-theoretic calculations and allows us to understand not only the behavior of the Higgs mass but also the overall stability of the string theory in a very compact form. Indeed, in some regions this potential exhibits explicitly string-theoretic behavior. However, in other regions, this potential-despite its string-theoretic origins-exhibits a number of features which are reminiscent of the traditional Coleman-Weinberg Higgs potential.

Finally, in Sec. VI, we provide an overall discussion of our results and outline some possibilities for future research. We also provide an additional bird's-eye perspective on the manner in which modular invariance induces UV/IR mixing and the reason why the passage from a full string-theoretic result to an EFT description necessarily breaks the modular symmetry. We will also discuss some of the possible implications of our results for addressing the hierarchy problems associated with the cosmological constant and the Higgs mass. This paper also has two appendices which provide the details of calculations whose results are quoted in Secs. IV E 1 and IV E 2 respectively.

Our overarching goal in this paper is to provide a fully string-theoretic framework for the calculation of the Higgs mass - a framework in which modular invariance is baked into the formalism from the very beginning. Our results can therefore potentially serve as the launching point for a rigorous investigation of the gauge hierarchy problem in string theory. However, our methods are quite general and can easily be adapted to other quantities of phenomenological interest, including not only the masses of all particles in the theory but also the gauge couplings, quartic couplings, and indeed the couplings associated with all allowed interactions.

As already noted, much of the inspiration for this work stems from our conviction that it is not an accident or phenomenological irrelevancy that string theories contain not only low-lying modes but also infinite towers of massive states. Together, all of these states conspire to enforce many of the unique symmetries for which string theory is famous, and thus their effects are an intrinsic part of the predictions of string theory. In this spirit, one might even view our work as a continuation of the line originally begun in the classic 1987 paper of Kaplunovsky [1] which established a framework for calculating string threshold corrections in which the contributions of the infinite towers of string states were included. Indeed, as discussed in Sec. IV C, some of our results for the Higgs mass even resemble results obtained in Ref. [1] for threshold corrections. One chief difference in our work, however, is our insistence on maintaining modular invariance at all steps in the calculation, including the regulators, especially when 
seeking to understand the behavior of dimensionful operators. It is this extra ingredient which is critical for ensuring consistency with the underlying string symmetries, and which allows us to probe the unique effects of such symmetries (such as those induced by UV/IR mixing) in a rigorous manner.

\section{MODULAR INVARIANCE AND THE HIGGS MASS: A GENERAL FRAMEWORK}

In this section we develop a framework for calculating the Higgs mass in any four-dimensional modular-invariant string theory. Our framework incorporates modular invariance in a fundamental way, and ultimately leads to a completely general expression for one-loop Higgs mass. Our results can therefore easily be applied to any fourdimensional closed-string model. Throughout most of this paper, our analysis will focus on heterotic-string models and will proceed under the assumption that the string model in question can be described through a corresponding charge lattice. As we shall see, the existence of a charge lattice provides a very direct way of performing our calculations and illustrating our main points. However, as we shall discuss in Sec. V, our results are ultimately more general than this, and apply even for closed-string models that transcend a specific charge-lattice construction.

\section{A. Preliminaries: String partition functions, charge lattices, and modular invariance}

We begin by reviewing basic facts about string partition functions, charge lattices, and modular invariance, establishing our notation and normalizations along the way. The one-loop partition function for any closed heterotic string in four spacetime dimensions is a statistics-weighted trace over the Fock space of closed-string states, and thus takes the general form

$$
\mathcal{Z}(\tau, \bar{\tau}) \equiv \tau_{2}^{-1} \frac{1}{\bar{\eta}^{12} \eta^{24}} \sum_{m, n}(-1)^{F} \bar{q}^{m} q^{n} .
$$

Here $\tau$ is the one-loop (torus) modular parameter, $\tau_{2} \equiv \operatorname{Im} \tau$, $q \equiv \exp (2 \pi i \tau), F$ is the spacetime fermion number, and the Dedekind eta-function is $\eta(\tau) \equiv q^{1 / 24} \prod_{n=1}^{\infty}\left(1-q^{n}\right)$. In this expression, the $\bar{\eta}$ and $\eta$ functions represent the contributions from the string oscillator states [which include appropriate right- and left-moving vacuum energies $(-1 / 2,-1)$ respectively], while the $(m, n)$ sum tallies the contributions from the Kaluza-Klein (KK) and winding excitations of the heterotic-string world sheet fieldsexcitations which result from the compactification of the heterotic string to four dimensions from its critical spacetime dimensions ( $=10$ for the right movers and 26 for the left movers), with $(m, n)$ representing the corresponding right- and left-moving world sheet energies. These $\mathrm{KK} /$ winding contributions can be written in terms of the charge vectors $\mathbf{Q} \equiv\left\{\mathbf{Q}_{R}, \mathbf{Q}_{L}\right\}$ of a $(10,22)$-dimensional Lorentzian charge lattice - or equivalently the $\mathrm{KK} /$ winding momenta $\left\{\mathbf{p}_{R}, \mathbf{p}_{L}\right\}$ of a corresponding momentum lattice of the same dimensionality_-via

$$
m=\frac{\mathbf{Q}_{R}^{2}}{2}=\frac{\alpha^{\prime} \mathbf{p}_{R}^{2}}{2}, \quad n=\frac{\mathbf{Q}_{L}^{2}}{2}=\frac{\alpha^{\prime} \mathbf{p}_{L}^{2}}{2},
$$

where $\alpha^{\prime} \equiv 1 / M_{s}^{2}$ with $M_{s}$ denoting the string scale. Thus the partition function in Eq. (2.1) can be written as a sum over charge vectors $\mathbf{Q}_{L}, \mathbf{Q}_{R}$ :

$$
\mathcal{Z}(\tau, \bar{\tau})=\tau_{2}^{-1} \frac{1}{\bar{\eta}^{12} \eta^{24}} \sum_{\mathbf{Q}_{L}, \mathbf{Q}_{R}}(-1)^{F} \bar{q}^{\mathbf{Q}_{R}^{2} / 2} q^{\mathbf{Q}_{L}^{2} / 2} .
$$

In general, the spacetime mass $M$ of the resulting string state is given by $\alpha^{\prime} M^{2}=2(m+n)+2\left(\Delta_{L}+\Delta_{R}\right)+2\left(a_{L}+a_{R}\right)$ where $\Delta_{R, L}$ are the contributions from the oscillator excitations and $\left(a_{R}, a_{L}\right)=(-1 / 2,-1)$ are the corresponding vacuum energies. Identifying individual left- and rightmoving contributions to $M^{2}$ through the convention

$$
M^{2}=\frac{1}{2}\left(M_{L}^{2}+M_{R}^{2}\right)
$$

then yields $\quad \alpha^{\prime} M_{R}^{2}=4\left(m+\Delta_{R}+a_{R}\right) \quad$ and $\quad \alpha^{\prime} M_{L}^{2}=$ $4\left(n+\Delta_{L}+a_{L}\right)$. Writing these masses in terms of the lattice charge vectors then yields

$$
\begin{aligned}
& \frac{\alpha^{\prime}}{2} M_{R}^{2}=\mathbf{Q}_{R}^{2}+2 \Delta_{R}+2 a_{R}, \\
& \frac{\alpha^{\prime}}{2} M_{L}^{2}=\mathbf{Q}_{L}^{2}+2 \Delta_{L}+2 a_{L} .
\end{aligned}
$$

States are level-matched (physical) if $M_{R}^{2}=M_{L}^{2}$ and unphysical otherwise. Indeed, with these conventions, gauge bosons in the left-moving non-Cartan algebra are massless, with $\mathbf{Q}_{L}^{2}=2$ and $\Delta_{L}=0$, while those in the left-moving Cartan algebra are massless, with $\mathbf{Q}_{L}^{2}=0$ and $\Delta_{L}=1$. (Indeed, such results apply to all left-moving simply laced gauge groups with level-one affine realizations; more complicated situations, such as necessarily arise for the rightmoving gauge groups, are discussed in Ref. [2].) Note that the $C P T$ conjugate of any state with charge vector $\left\{\mathbf{Q}_{R}, \mathbf{Q}_{L}\right\}$ has charge vector $-\left\{\mathbf{Q}_{R}, \mathbf{Q}_{L}\right\}$. Thus $C P T$ invariance requires that all states in the string spectrum come in $\pm\left\{\mathbf{Q}_{R}, \mathbf{Q}_{L}\right\}$ pairs. By contrast, since the right-moving gauge group is necessarily nonchiral as a result of superconformality constraints, the chiral conjugate of any state with charge vector $\left\{\mathbf{Q}_{R}, \mathbf{Q}_{L}\right\}$ has charge vector $\left\{\mathbf{Q}_{R},-\mathbf{Q}_{L}\right\}$.

One important general property of the partition functions in Eq. (2.1) — and indeed the partition functions of all closed strings in any spacetime dimension-is that they must be modular invariant, i.e., invariant under all transformations of the form $\tau \rightarrow(a \tau+b) /(c \tau+d)$ where 
$a, b, c, d \in \mathbb{Z}$ and $a d-b c=1$ (with the same transformation for $\bar{\tau}$ ). Modular invariance is thus an exact symmetry underpinning all heterotic strings, and in this paper we shall be exploring its consequences for the masses of the Higgs fields in such theories. For these purposes, it will be important to understand the manner in which these partition functions achieve their modular invariance. In general, the partition functions for heterotic strings in four dimensions can be rewritten in the form

$$
\mathcal{Z}(\tau, \bar{\tau}) \equiv \tau_{2}^{-1} \frac{1}{\bar{\eta}^{12} \eta^{24}} \sum_{\bar{i}, i} N_{\bar{i} i} \overline{g_{\bar{\imath}}(\tau)} f_{i}(\tau)
$$

where each $(\bar{l}, i)$ term represents the contribution from a different sector of the theory and where the left-moving holomorphic $f_{i}$ functions (and the corresponding rightmoving antiholomorphic $g_{\bar{l}}$ functions) transform covariantly under modular transformations according to relations of the form

$$
f\left(\frac{a \tau+b}{c \tau+d}\right) \sim(c \tau+d)^{k} f(\tau)
$$

where $k$ is the so-called modular weight of the $f_{i}$ functions (with an analogous weight $\bar{k}$ for the $g_{\bar{\imath}}$ functions) and where the $\sim$ notation allows for the possibility of overall $\tau$-independent phases which will play no future role in our arguments. We likewise have

$$
\eta\left(\frac{a \tau+b}{c \tau+d}\right) \sim(c \tau+d)^{1 / 2} \eta(\tau) .
$$

Thus, since $\tau_{2} \rightarrow \tau_{2} /|c \tau+d|^{2}$ as $\tau \rightarrow(a \tau+b) /(c \tau+d)$, we immediately see that modular invariance of the entire partition function in Eq. (2.6) requires not only that the $N_{\bar{u} i}$ coefficients in Eq. (2.6) be chosen correctly but also that $k=11$ and $\bar{k}=5$. In general, for strings realizable through free-field constructions, these $f_{i}$ and $g_{\bar{l}}$ functions produce the lattice sum in Eq. (2.3) because they can be written in the factorized forms

$$
f_{i} \sim \prod_{\ell=1}^{22} \vartheta\left[\begin{array}{c}
\alpha_{\ell}^{(i)} \\
\beta_{\ell}^{(i)}
\end{array}\right], \quad g_{\bar{\imath}} \sim \prod_{\ell=1}^{10} \vartheta\left[\begin{array}{c}
\alpha_{\ell}^{(\bar{i})} \\
\beta_{\ell}^{(\bar{l})}
\end{array}\right],
$$

where each $\vartheta$-function factor is the trace over the $\ell$ th direction $Q_{\ell}$ of the charge lattice:

$$
\vartheta_{\ell}(\tau) \equiv \vartheta\left[\begin{array}{l}
\alpha_{\ell} \\
\beta_{\ell}
\end{array}\right](\tau) \equiv \sum_{Q_{\ell} \in \mathbb{Z}+\alpha_{\ell}} e^{2 \pi i \beta_{\ell} Q_{\ell}} q^{Q_{\ell}^{2} / 2}
$$

Indeed, the $\vartheta_{\ell}$ functions transform under modular transformations as in Eq. (2.7), with modular weight $1 / 2$. The modular invariance of the underlying string theory then ensures that there exists a special $(10,22)$-dimensional "spin-statistics vector" $\mathbf{S}$ such that we may identify the spacetime fermion number $F$ within Eq. (2.1) as $F=2 \mathbf{Q} \cdot \mathbf{S}$ $(\bmod 2)$ for any state with charge $\mathbf{Q}$, where the dot notation "." signifies the Lorentzian (left-moving minus right-moving) dot product. Modular invariance also implies that the shifted charges $\mathbf{Q}-\mathbf{S}$ associated with the allowed string states together form a Lorentzian lattice which is both odd and self-dual. It is with this understanding that we refer to the charges $\mathbf{Q}$ themselves as populating a "lattice." Indeed, it is the self-duality property of the shifted charge lattice $\{\mathbf{Q}-$ S $\}$ which guarantees that the $f_{i}$ and $g_{\bar{l}}$ functions in Eq. (2.7) transform covariantly under the modular group, as in Eq. (2.7).

For later purposes, we simply observe that the general structure given in Eq. (2.6) is typical of the modularinvariant quantities that arise as heterotic-string Fock-space traces. Indeed, a general quantity of the form

$$
\tau_{2}^{\kappa} \frac{1}{\bar{\eta}^{12} \eta^{24}} \sum_{\bar{i}, i} N_{\bar{i}} \overline{g_{\bar{i}}(\tau)} f_{i}(\tau)
$$

cannot be modular invariant unless the $N_{\overline{l i}}$ are chosen correctly and the corresponding $f_{i}$ and $g_{\bar{l}}$ functions transform as in Eq. (2.7) with

$$
k-12=\bar{k}-6=\kappa .
$$

While $\kappa=-1$ for the partition functions of fourdimensional heterotic strings, as described above, we shall see that other important Fock-space traces can have different values of $\kappa$. For example, the partition functions of heterotic strings in $D$ spacetime dimensions have $\kappa=1-D / 2$, with corresponding changes to the dimensionalities of their associated charge lattices.

\section{B. Higgsing and charge-lattice deformations}

In general, different string models exhibit different spectra and thus have different charge lattices. However, Higgsing a theory changes its spectrum in certain dramatic ways, such as by giving mass to formerly massless gauge bosons and thereby breaking the associated gauge symmetries. Thus, in string theory, Higgsing can ultimately be viewed as a process of transforming the charge lattice from one configuration to another.

Of course, modular invariance must be maintained throughout the Higgsing process. Indeed, it is only in this way that we can regard the Higgsing process as a fully stringtheoretic operation that shifts the string vacuum state within the space of self-consistent string vacua. However, modular invariance then implies that the charge-lattice transformations induced by Higgsing are not arbitrary. Instead, they must preserve those charge-lattice properties, as described above, which guarantee the modular invariance of the theory.

This in turn tells us that the process of Higgsing is likely to be far more complicated in string theory than it is in 
ordinary quantum field theory. In general, the charge lattice receives contributions from all sectors of the theory, and modular transformations mix these different contributions in highly nontrivial ways. Thus the process of Higgsing a given gauge symmetry within a given sector of a string model generally involves not only the physics associated with that gauge symmetry but also the physics of all of the other sectors of the theory as well, both twisted and untwisted, and the properties of the other gauge symmetries, including gravity, that might also be present in the string model-even if these other gauge symmetries are apparently completely disjoint from the symmetry being Higgsed. We shall see explicit examples of this below. Moreover, in string theory the dynamics of the Higgs vacuum expectation value (VEV)—and indeed the dynamics of all string moduli-is generally governed by an effective potential which is nothing but the vacuum energy of the theory, expressed as a function of this VEV. Thus the overall dynamics associated with Higgsing can be rather subtle: the Higgs VEV determines the deformations of the charge lattice, and these deformations alter the vacuum energy which in turn determines the VEV.

In this paper, our goal is to calculate the mass of the physical Higgs scalar field that emerges in the Higgsed phase (i.e., after the theory has already been Higgsed). We shall therefore assume that our theory contains a scalar Higgs field which has already settled into the new minimum of its potential. This will allow us to sidestep the (rather complex) model-dependent issue concerning the manner in which the Higgsing itself occurs, and instead focus on the perturbations of the field around this new minimum. In this way we will be able to determine the curvature of the scalar potential at this local minimum, and thereby obtain the corresponding Higgs mass.

To do this, we shall begin by exploring the manner in which a general charge lattice is deformed as we vary a scalar Higgs field away from the minimum of its potential. Our discussion will be completely general, and we shall defer to Sec. II C any assumptions that might be specific to the particular Higgs field responsible for electroweak symmetry breaking. For concreteness, we shall let our scalar field have a value $\langle\phi\rangle+\phi$, where $\langle\phi\rangle$ is the Higgs $\mathrm{VEV}$ at the minimum of its potential and where $\phi$ describes the fluctuations away from this point. If $\left\{\mathbf{Q}_{L}, \mathbf{Q}_{R}\right\}$ are the charge vectors associated with a given string state in the Higgsed phase (i.e., at the minimum of the potential, when $\phi=0$ ), then turning on $\phi$ corresponds to deforming these charge vectors. In general, for $\phi /\langle\phi\rangle \ll 1$, we shall parametrize these deformations according to

$$
\begin{aligned}
\mathbf{Q}_{L} & \rightarrow \mathbf{Q}_{L}+\sqrt{\alpha^{\prime}} \phi \mathbf{Q}_{a}+\frac{1}{2} \alpha^{\prime} \phi^{2} \mathbf{Q}_{b}+\ldots \\
\mathbf{Q}_{R} & \rightarrow \mathbf{Q}_{R}+\sqrt{\alpha^{\prime}} \phi \tilde{\mathbf{Q}}_{a}+\frac{1}{2} \alpha^{\prime} \phi^{2} \tilde{\mathbf{Q}}_{b}+\ldots
\end{aligned}
$$

where $\mathbf{Q}_{a}, \mathbf{Q}_{b}, \tilde{\mathbf{Q}}_{a}$, and $\tilde{\mathbf{Q}}_{b}$ are deformation charge vectors of dimensionalities 22, 22, 10, and 10 respectively. Indeed, the forms of these vectors are closely correlated with the specific gauge symmetries broken by the Higgsing process, and as such these vectors continue to govern the fluctuations of the Higgs scalar around this Higgsed minimum.

In this paper, we shall keep our analysis as general as possible. As such, we shall not make any specific assumptions regarding the forms of these vectors. However, as discussed above, we know that the Higgsing process - and even the fluctuations around the Higgsed minimum of the potential-should not break modular invariance. In particular, the corresponding charge-lattice deformations in Eq. (2.13) should not disturb level-matching. This means that the value of the difference $\mathbf{Q}_{L}^{2}-\mathbf{Q}_{R}^{2}$ should not be disturbed when $\phi$ is taken to nonzero values, which in turn means that this difference should be independent of $\phi$. This then constrains the choices for the vectors $\mathbf{Q}_{a}, \mathbf{Q}_{b}, \tilde{\mathbf{Q}}_{a}$, and $\tilde{\mathbf{Q}}_{b}$.

To help simplify the notation, let us assemble a single 32-dimensional charge vector $\mathbf{Q} \equiv\left(\mathbf{Q}_{L}, \mathbf{Q}_{R}\right)^{t}$ (where " $t$ " signifies the transpose). Recalling that the dot notation "." signifies the Lorentzian (left-moving minus right-moving) contraction of vector indices, as appropriate for a Lorentzian charge lattice, we therefore require that $\mathbf{Q}^{2} \equiv$ $\mathbf{Q}^{t} \cdot \mathbf{Q}$ be $\phi$-independent for all $\phi$. Given the above shifts, we find that terms within $\mathbf{Q}^{t} \cdot \mathbf{Q}$ which are respectively linear and quadratic in $\phi$ will cancel provided

$$
\begin{array}{r}
\left(\mathbf{Q}_{a}^{t}, \tilde{\mathbf{Q}}_{a}^{t}\right) \cdot \mathbf{Q}=0 \\
\left(\mathbf{Q}_{b}^{t}, \tilde{\mathbf{Q}}_{b}^{t}\right) \cdot \mathbf{Q}+\left(\mathbf{Q}_{a}^{t}, \tilde{\mathbf{Q}}_{a}^{t}\right) \cdot\left(\begin{array}{c}
\mathbf{Q}_{a} \\
\tilde{\mathbf{Q}}_{a}
\end{array}\right)=0
\end{array}
$$

These are thus modular-invariance constraints on the allowed choices for the shift vectors $\mathbf{Q}_{a}, \mathbf{Q}_{b}, \tilde{\mathbf{Q}}_{a}$, and $\tilde{\mathbf{Q}}_{b}$.

We can push these constraints one step further if we write these shift vectors in terms of $\mathbf{Q}$ itself via relations of the form

$$
\left(\begin{array}{c}
\mathbf{Q}_{a} \\
\tilde{\mathbf{Q}}_{a}
\end{array}\right)=\mathcal{T} \cdot \mathbf{Q}, \quad\left(\begin{array}{c}
\mathbf{Q}_{b} \\
\tilde{\mathbf{Q}}_{b}
\end{array}\right)=\mathcal{N} \cdot \mathbf{Q}
$$

where $\mathcal{T}$ and $\mathcal{N}$ are $(32 \times 32)$-dimensional matrices and where "." retains its Lorentzian signature for the index contraction that underlies matrix multiplication. The first constraint equation above then tell us that $\mathbf{Q}^{t} \cdot \mathcal{T} \cdot \mathbf{Q}=0$, which implies that $\mathcal{T}$ must be antisymmetric, while the second constraint equation tells us that $\mathbf{Q}^{t} \cdot\left(\mathcal{N}+\mathcal{T}^{t} \cdot \mathcal{T}\right)$. $\mathbf{Q}=0$, which implies that $\mathcal{N}+\mathcal{T}^{t} \cdot \mathcal{T}$ must also be antisymmetric. It turns out that the precise value of $\mathcal{N}+\mathcal{T}^{t} \cdot \mathcal{T}$ will have no bearing on the Higgs mass. We will therefore set it to zero (which is indeed antisymmetric), implying that $\mathcal{N}=-\mathcal{T}^{t} \cdot \mathcal{T}$. Thus, while $\mathcal{T}$ is antisymmetric, $\mathcal{N}$ is symmetric. Indeed, if we write our 
$\mathcal{T}$-matrix in terms of left- and right-moving submatrices $\mathcal{T}_{i j}$ in the form

$$
\mathcal{T}=\left(\begin{array}{ll}
\mathcal{T}_{11} & \mathcal{T}_{12} \\
\mathcal{T}_{21} & \mathcal{T}_{22}
\end{array}\right)
$$

then we must have $\mathcal{T}_{11}^{t}=-\mathcal{T}_{11}, \mathcal{T}_{22}^{t}=-\mathcal{T}_{22}$, and $\mathcal{T}^{t}{ }_{12}=-\mathcal{T}_{21}$. Likewise, we then find that

$$
\begin{aligned}
& \mathcal{N}_{11}=-\mathcal{T}_{11}^{t} \mathcal{T}_{11}+\mathcal{T}_{21}^{t} \mathcal{T}_{21}, \\
& \mathcal{N}_{12}=-\mathcal{T}_{11}^{t} \mathcal{T}_{12}+\mathcal{T}_{21}^{t} \mathcal{T}_{22}, \\
& \mathcal{N}_{21}=-\mathcal{T}_{12}^{t} \mathcal{T}_{11}+\mathcal{T}_{22}^{t} \mathcal{T}_{21}, \\
& \mathcal{N}_{22}=-\mathcal{T}_{12}^{t} \mathcal{T}_{12}+\mathcal{T}_{22}^{t} \mathcal{T}_{22} .
\end{aligned}
$$

\section{Example: The Standard Model Higgs}

In general, within any given string model, the deformation vectors $\mathbf{Q}_{a}, \mathbf{Q}_{b}, \tilde{\mathbf{Q}}_{a}$, and $\tilde{\mathbf{Q}}_{b}$ in Eq. (2.13) depend on the particular charge vector $\left(\mathbf{Q}_{R}, \mathbf{Q}_{L}\right)$ being deformed. However the $\mathcal{T}$ - and $\mathcal{N}$-matrices in Eq. (2.15) are universal for all charge vectors within the model. It is therefore these matrices which carry all of the relevant information concerning the response of the theory to fluctuations of the particular Higgs field under study. In general, these matrices depend on how the gauge groups and corresponding Higgs field are embedded within the charge lattice. Thus the precise forms of these matrices depend on the particular string model under study and the Higgs field to which it gives rise.

To illustrate this point, it may be helpful to consider the special case of the Standard Model (SM) Higgs. For concreteness, we shall work within the framework of heterotic string models in which the Standard Model itself is realized at affine level $k=1$ through a standard level-one $S O(10)$ embedding. In the following we shall adhere to the conventions in Ref. [3]. Since $S O(10)$ has rank 5, this group can be minimally embedded within a fivedimensional sublattice $\left\{Q_{1}, Q_{2}, Q_{3}, Q_{4}, Q_{5}\right\}$ within the full 22-dimensional left-moving lattice $\left\{\mathbf{Q}_{L}\right\}$. Within this sublattice, we shall take the $\ell=1,2$ directions as corresponding to the $U(2)=S U(2) \times U(1)$ electroweak subgroup of $S O(10)$, while the $\ell=3,4,5$ directions will correspond to the $U(3)=S U(3) \times U(1)$ color subgroup. By convention we will take the $S U(2)_{L}$ representations to lie along the line perpendicular to $(1,1,0,0,0)$ within the two-dimensional $U(2)$ sublattice, and the $S U(3)_{c}$ representations to lie within the two-dimensional plane perpendicular to $(0,0,1,1,1)$ within the three-dimensional $U(3)$ sublattice. It then follows that any state with charge vector $\mathbf{Q}_{L}$ has $S U(2)$ quantum numbers determined by projecting $\mathbf{Q}_{L}$ onto the $S U(2)$ line [thereby yielding an $S U(2)$ weight in the corresponding $S U(2)$ weight system] and $S U(3)$ quantum numbers determined by projecting $\mathbf{Q}_{L}$ onto the $S U(3)$ plane [thereby yielding an $S U(3)$ weight within the corresponding $S U(3)$ weight system]. Likewise, the $S O(10)$-normalized hypercharge $Y$ of any state with left-moving charge vector $\mathbf{Q}_{L}$ is given by $Y=$ $\sum_{\ell=1}^{5} a_{Y}^{(\ell)} Q_{\ell}$ where

$$
\mathbf{a}_{Y}=\left(\frac{1}{2}, \frac{1}{2},-\frac{1}{3},-\frac{1}{3},-\frac{1}{3}\right)
$$

(with all other components vanishing). Thus, $Y \equiv \mathbf{a}_{Y} \cdot \mathbf{Q}_{L}$. Indeed we see that $k_{Y} \equiv 2 \mathbf{a}_{Y} \cdot \mathbf{a}_{Y}=5 / 3$, as appropriate for the standard $S O(10)$ embedding [as well as other nonstandard $S O(10)$ embeddings [3]]. In a similar way, the electromagnetic charge $q_{\mathrm{EM}}$ of any state with charge vector $\mathbf{Q}_{L}$ is given by $q_{\mathrm{EM}}=\mathbf{a}_{\mathrm{EM}} \cdot \mathbf{Q}_{L}$, where

$$
\mathbf{a}_{\mathrm{EM}}=\left(0,1,-\frac{1}{3},-\frac{1}{3},-\frac{1}{3}\right)
$$

(with all other components vanishing). As a check we verify that $T_{3}=\mathbf{a}_{T_{3}} \cdot \mathbf{Q}_{L}$, where $\mathbf{a}_{T_{3}}=\mathbf{a}_{\mathrm{EM}}-\mathbf{a}_{Y}=$ $\left(-\frac{1}{2}, \frac{1}{2}, 0,0,0\right)=\frac{1}{2} \mathbf{Q}_{T^{+}}$where $\mathbf{Q}_{T^{+}}$is the charge vector (or root vector) associated with the $S U(2)$ gauge boson with positive $T_{3}$ charge.

Thus far, we have focused on the gauge structure of the theory. As we have seen, the corresponding charge vectors follow our usual group-theoretic expectations, just as they would in ordinary quantum field theory. However, the charge vectors associated with the SM matter states in string theory are far more complex than would be expected in quantum field theory and actually spill beyond the $S O(10)$ sublattice.

To see why this is so, it is perhaps easiest to consider the original $S O(10)$ theory prior to electroweak breaking. In this phase of the theory, the SM matter content consists of massless fermion and Higgs fields transforming in the $\mathbf{1 6}$ and 10 representations of $S O(10)$, respectively. The former representations has charge vectors with $S O(10)$-sublattice components $Q_{\ell}^{(f)}= \pm 1 / 2$ for each $\ell$ (with an odd net number of minus signs), while the latter has $Q_{\ell}^{(\phi)}= \pm \delta_{\ell k}$ where $k=1,2, \ldots, 5$. Thus, the $\mathbf{1 6}$ and $\mathbf{1 0}$ representations have conformal dimensions $h_{16}=5 / 8$ and $h_{10}=1 / 2$. Indeed, according to the gauge embeddings discussed above, the particular Higgs states which are electrically neutral have $Q_{\ell}= \pm \delta_{\ell 1}$. However, as a result of the nontrivial left-moving heterotic-string vacuum energy $E_{L}=-1$, any massless string state must correspond to world sheet excitations contributing a total left-moving conformal dimension $h_{L}=1$. Thus, even within the $S O(10)$ embedding specified above, string consistency constraints require that the SM fermion and Higgs states carry nontrivial charges not only within the $S O(10)$ sublattice $\left\{Q_{1}, Q_{2}, \ldots, Q_{5}\right\}$ but also beyond it-i.e., elsewhere in the 17 remaining left-moving lattice directions 
$\mathbf{Q}_{\text {int }} \equiv\left\{Q_{6}, \ldots, Q_{22}\right\}$ which a priori correspond to gauge symmetries beyond those of the SM (such as those of potential hidden sectors). Indeed, these additional excitations must contribute additional left-moving conformal dimensions $3 / 8$ and $1 / 2$ for the SM matter and Higgs fields respectively, corresponding to $\left[\mathbf{Q}_{\text {int }}^{(f)}\right]^{2}=3 / 4$ for the fermions and $\left[\mathbf{Q}_{\mathrm{int}}^{(\phi)}\right]^{2}=1$ for the Higgs.

A similar phenomenon also occurs within the tendimensional right-moving charge lattice, with components $\left\{\tilde{Q}_{1}, \ldots, \tilde{Q}_{10}\right\}$. The component associated with the nonzero component of the $\mathbf{S}$-vector discussed below Eq. (2.10) henceforth chosen as $\tilde{Q}_{1}$-describes the spacetime spinhelicity of the state. As such, we must have $\tilde{Q}_{1}^{(f)}= \pm 1 / 2$ for the SM fermions and $\tilde{Q}_{1}^{(\phi)}=0$ for the scalar Higgs. Of course, the right-moving side of the heterotic string has $E_{R}=-1 / 2$, requiring that all massless string states have total right-moving conformal dimensions $h_{R}=1 / 2$. We thus find that the SM fermion and Higgs fields must have additional nine-dimensional charge vectors $\tilde{\mathbf{Q}}_{\text {int }} \equiv$ $\left\{\tilde{Q}_{2}, \ldots, \tilde{Q}_{10}\right\}$ (presumably corresponding to additional right-moving gauge symmetries) such that $\left[\tilde{\mathbf{Q}}_{\text {int }}^{(f)}\right]^{2}=3 / 4$ and $\left[\tilde{\mathbf{Q}}_{\text {int }}^{(\phi)}\right]^{2}=1$.

We see, then, that the electrically neutral Higgs field prior to electroweak symmetry breaking must have a total 32-dimensional charge vector of the form

$$
\begin{aligned}
\mathbf{Q}_{\phi} & \equiv\left(\mathbf{Q}_{L}^{(\phi)} \mid \mathbf{Q}_{R}^{(\phi)}\right) \\
& =\left(1,0,0,0,0, \mathbf{Q}_{\text {int }}^{(\phi)} \mid 0, \tilde{\mathbf{Q}}_{\text {int }}^{(\phi)}\right)
\end{aligned}
$$

where $\left[\mathbf{Q}_{\text {int }}^{(\phi)}\right]^{2}=\left[\tilde{\mathbf{Q}}_{\text {int }}^{(\phi)}\right]^{2}=1$. In general, the specific forms of $\mathbf{Q}_{\text {int }}^{(\phi)}$ and $\tilde{\mathbf{Q}}_{\text {int }}^{(\phi)}$ depend on the specific string model and the spectrum beyond the Standard Model. However, those components which are specified within Eq. (2.20) are guaranteed by the underlying $S O(10)$ structure and by the requirement that the Higgs be electrically neutral. Of course, the process of electroweak symmetry breaking can in principle alter the form of this vector. However, we know that $U(1)_{\mathrm{EM}}$ necessarily remains unbroken. Thus, even if the forms of the particular "internal" vectors $\mathbf{Q}_{\text {int }}^{(\phi)}$ and $\tilde{\mathbf{Q}}_{\mathrm{int}}^{(\phi)}$ are shifted under electroweak symmetry breaking, the zeros in the charge vector in Eq. (2.20) ensure the electric neutrality of the Higgs field and must therefore be preserved. This remains true not only for the physical Higgs field after electroweak symmetry breaking, but also for its quantum fluctuations in the Higgsed phase.

This observation immediately allows us to constrain the form of the $\mathcal{T}$-matrices which parametrize the response of the charge lattice to small fluctuations of the Higgs field around its minimum. Because the zeros in the charge vector in Eq. (2.20) must remain vanishing - and indeed because the electromagnetic charges and spin-statistics of all string states must remain unaltered under such fluctuations-we see that the (necessarily antisymmetric) $\mathcal{T}$-matrix can at most have the general form

$\mathcal{T} \sim\left(\begin{array}{cccccc|cc} & & & & & \mathbf{t} & 0 & \tilde{\mathbf{t}} \\ & & & & & \mathbf{0} & 0 & \tilde{\mathbf{0}} \\ & & \mathbf{0}_{5 \times 5} & & & \mathbf{0} & 0 & \tilde{\mathbf{0}} \\ & & & & & \mathbf{0} & 0 & \tilde{\mathbf{0}} \\ & & & & & \mathbf{0} & 0 & \tilde{\mathbf{0}} \\ -\mathbf{t}^{t} & \mathbf{0}^{t} & \mathbf{0}^{t} & \mathbf{0}^{t} & \mathbf{0}^{t} & \mathbf{t}_{11} & \mathbf{0}^{t} & \mathbf{t}_{12} \\ \hline 0 & 0 & 0 & 0 & 0 & \mathbf{0} & 0 & \tilde{\mathbf{0}} \\ -\tilde{\mathbf{t}}^{t} & \tilde{\mathbf{0}}^{t} & \tilde{\mathbf{0}}^{t} & \tilde{\mathbf{0}}^{t} & \tilde{\mathbf{0}}^{t} & -\mathbf{t}_{12}^{t} & \tilde{\mathbf{0}}^{t} & \mathbf{t}_{22}\end{array}\right)$

where $\mathbf{t}$ is an arbitrary 17-dimensional row vector; where $\tilde{\mathbf{t}}$ is an arbitrary nine-dimensional row vector; where $\mathbf{t}_{11}, \mathbf{t}_{12}$, and $\mathbf{t}_{22}$ are arbitrary matrices of dimensionalities $17 \times 17,9 \times 17$, and $9 \times 9$, respectively, with $\mathbf{t}_{11}$ and $\mathbf{t}_{22}$ antisymmetric; and where $\mathbf{0}$ and $\tilde{\mathbf{0}}$ are respectively 17 - and 9-dimensional zero row vectors. Indeed, as we have seen, only this form of the $\mathcal{T}$-matrix can preserve the electromagnetic charges and spin-statistics of the string states under small shifts in the Higgs field around its new minimum, assuming a heterotic string model with a standard level-one $S O(10)$ embedding. The precise forms of $\mathbf{t}, \tilde{\mathbf{t}}, \mathbf{t}_{11}, \mathbf{t}_{12}$, and $\mathbf{t}_{22}$ then depend on more model-specific details of how the Higgs is realized within the theorydetails which go beyond the $S O(10)$ embedding.

As indicated above, this is only one particular example of the kinds of $\mathcal{T}$-matrices that can occur. However, all of the results of this paper will be completely general, and will not rest on this particular example.

\section{Calculating the Higgs mass}

We can now use the general results in Sec. II B to calculate the mass of $\phi$. In general, this mass can be defined as

$$
\left.m_{\phi}^{2} \equiv \frac{d^{2} \Lambda(\phi)}{d \phi^{2}}\right|_{\phi=0}
$$

where

$$
\Lambda(\phi) \equiv-\frac{\mathcal{M}^{4}}{2} \int_{\mathcal{F}} \frac{d^{2} \tau}{\tau_{2}^{2}} \mathcal{Z}(\tau, \bar{\tau}, \phi)
$$

Indeed, $\Lambda(\phi)$ is the vacuum energy that governs the dynamics of $\phi$. Here $d^{2} \tau / \tau_{2}^{2}$ is the modular-invariant integration measure, $\mathcal{F}$ is the fundamental domain of the modular group

$$
\mathcal{F} \equiv\left\{\tau:-\frac{1}{2}<\tau_{1} \leq \frac{1}{2}, \tau_{2}>0,|\tau| \geq 1\right\},
$$


and $\mathcal{M} \equiv M_{s} /(2 \pi)$ is the reduced string scale. In this expression, following Eq. (2.3), the shifted partition function is given by

$$
\mathcal{Z}(\tau, \bar{\tau}, \phi)=\tau_{2}^{-1} \frac{1}{\bar{\eta}^{12} \eta^{24}} \sum_{\mathbf{Q}_{L}, \mathbf{Q}_{R}}(-1)^{F} \bar{q}^{\mathbf{Q}_{R}^{2} / 2} q^{\mathbf{Q}_{L}^{2} / 2}
$$

where the left- and right-moving charge vectors $\mathbf{Q}_{L}$ and $\mathbf{Q}_{R}$ are now deformed as in Eq. (2.13) and thus depend on $\phi$.

Given this definition, we begin by evaluating the leading contribution to the Higgs mass by taking partial derivatives of $\mathcal{Z}$, i.e.,

$$
\frac{\partial^{2} \mathcal{Z}}{\partial \phi^{2}}=\tau_{2}^{-1} \frac{1}{\bar{\eta}^{12} \eta^{24}} \sum_{\mathbf{Q}_{L}, \mathbf{Q}_{R} \in L}(-1)^{F} X \bar{q}^{\mathbf{Q}_{R}^{2} / 2} q^{\mathbf{Q}_{L}^{2} / 2}
$$

where the summand insertion $X$ is given by

$$
X \equiv \pi i \frac{\partial^{2}}{\partial \phi^{2}}\left(\tau \mathbf{Q}_{L}^{2}-\bar{\tau} \mathbf{Q}_{R}^{2}\right)-\pi^{2}\left[\frac{\partial}{\partial \phi}\left(\tau \mathbf{Q}_{L}^{2}-\bar{\tau} \mathbf{Q}_{R}^{2}\right)\right]^{2} .
$$

Note that it is the partial derivative $\partial^{2} / \partial \phi^{2}$ in Eq. (2.26) which provides the leading contribution to the Higgs mass; we shall return to this point shortly. Expanding $X$ in powers of $\tau_{1}$ and $\tau_{2}$ and then setting $\phi=0$ yields

$$
\left.X\right|_{\phi=0}=A \tau_{1}+B \tau_{2}+C \tau_{1}^{2}+D \tau_{2}^{2}+E \tau_{1} \tau_{2},
$$

where

$$
\begin{aligned}
A & =0, \\
B & =-2 \pi \alpha^{\prime}\left(\mathbf{Q}_{a}^{2}+\tilde{\mathbf{Q}}_{a}^{2}+\mathbf{Q}_{b}^{t} \mathbf{Q}_{L}+\tilde{\mathbf{Q}}_{b}^{t} \mathbf{Q}_{R}\right), \\
C & =0 \\
D & =4 \pi^{2} \alpha^{\prime}\left(\mathbf{Q}_{a}^{t} \mathbf{Q}_{L}+\tilde{\mathbf{Q}}_{a}^{t} \mathbf{Q}_{R}\right)^{2}, \\
E & =0 .
\end{aligned}
$$

Note that $A, C$, and $E$ each vanish as the result of the constraints in Eq. (2.14). This is consistent, as these are the quantities which are proportional to powers of $\tau_{1}$, which multiplies $\mathbf{Q}_{L}^{2}-\mathbf{Q}_{R}^{2}$ within Eq. (2.27).

Using Eqs. (2.15) and (2.17), we can now express the shift vectors within Eq. (2.29) directly in terms of $\mathbf{Q}_{L}$ and $\mathbf{Q}_{R}$. For convenience we define

$$
\mathbf{Q}_{h} \equiv \mathcal{T}_{21} \mathbf{Q}_{L}, \quad \tilde{\mathbf{Q}}_{h} \equiv \mathcal{T}_{12} \mathbf{Q}_{R},
$$

and likewise define

$$
\mathbf{Q}_{j} \equiv \mathcal{T}_{11} \mathbf{Q}_{L}, \quad \tilde{\mathbf{Q}}_{j} \equiv \mathcal{T}_{22} \mathbf{Q}_{R}
$$

We then find

$$
\begin{aligned}
& B=-4 \pi \alpha^{\prime}\left(\mathbf{Q}_{h}^{2}+\tilde{\mathbf{Q}}_{h}^{2}-\tilde{\mathbf{Q}}_{j}^{t} \mathbf{Q}_{h}-\mathbf{Q}_{j}^{t} \tilde{\mathbf{Q}}_{h}\right), \\
& D=4 \pi^{2} \alpha^{\prime}\left(\mathbf{Q}_{R}^{t} \mathbf{Q}_{h}-\mathbf{Q}_{L}^{t} \tilde{\mathbf{Q}}_{h}\right)^{2} .
\end{aligned}
$$

Note the identity $\mathbf{Q}_{R}^{t} \mathbf{Q}_{h}=-\mathbf{Q}_{L}^{t} \tilde{\mathbf{Q}}_{h}$, as a result of which our expression for $D$ can actually be collapsed into one term. However, we have retained this form for $D$ in order to make manifest the symmetry between left- and right-moving contributions. Our overall insertion into the partition function is then given by $\left.X\right|_{\phi=0} \equiv \mathcal{X} / \mathcal{M}^{2}$, where

$$
\begin{aligned}
\mathcal{X}= & \tau_{2}^{2}\left(\mathbf{Q}_{R}^{t} \mathbf{Q}_{h}-\mathbf{Q}_{L}^{t} \tilde{\mathbf{Q}}_{h}\right)^{2} \\
& -\frac{\tau_{2}}{\pi}\left(\mathbf{Q}_{h}^{2}+\tilde{\mathbf{Q}}_{h}^{2}-\tilde{\mathbf{Q}}_{j}^{t} \mathbf{Q}_{h}-\mathbf{Q}_{j}^{t} \tilde{\mathbf{Q}}_{h}\right) .
\end{aligned}
$$

\section{E. Modular completion and additional Higgs-mass contributions}

Thus far, we have calculated the leading contribution to the Higgs mass by evaluating $\partial^{2} \mathcal{Z} / \partial \phi^{2}$. However, the full contribution $d^{2} \mathcal{Z} / d \phi^{2}$ (with full rather than partial $\phi$-derivatives) also includes various additional effects on the partition function $\mathcal{Z}$ that come from fluctuations of the Higgs field. For example, such fluctuations deform the background moduli fields (such as the metric that contracts compactified components of $\mathbf{Q}_{L}^{2}$ and $\mathbf{Q}_{R}^{2}$ within the charge lattice). Such effects produce additional contributions to the total Higgs mass.

It turns out that we can calculate all of these extra contributions in a completely general way through the requirement of modular invariance. Indeed, because modular invariance remains unbroken even when the theory is Higgsed, the final expression for the total Higgs mass must not only be modular invariant but also arise through a modular-covariant sequence of calculational operations. As we shall demonstrate, the above expression for the insertion $\mathcal{X}$ in Eq. (2.33) does not have this property. We shall therefore determine the additional contributions to the Higgs mass by performing the "modular completion" of $\mathcal{X}$-i.e., by determining the additional contribution to $\mathcal{X}$ which will render this insertion consistent with modular invariance.

In general, prior to the insertion of $\mathcal{X}$, the partition-function trace in Eq. (2.3) [or equivalently the trace in Eq. (2.25) evaluated at $\phi=0$ ] is presumed to already be modular invariant, as required for the consistency of the underlying string. In order to determine the modular completion of the quantity $\mathcal{X}$ in Eq. (2.33), we therefore need to understand the modular-invariance effects that arise when $\mathcal{X}$ is inserted into this partition-function trace. Because $\mathcal{X}$ involves various combinations of components of charge vectors, let us begin by investigating the effect of inserting powers of a single charge vector component $Q_{\ell}$ (associated with the $\ell$ th lattice direction) into our partition-function trace. Within the partition functions described in Eqs. (2.6) and (2.9), inserting $Q_{\ell}^{n}$ for any power $n$ is tantamount to replacing 


$$
\vartheta_{\ell} \rightarrow \sum_{Q_{\ell} \in \mathbb{Z}+\alpha_{\ell}} e^{2 \pi i \beta_{\ell} Q_{\ell}} Q_{\ell}^{n} q^{Q_{\ell}^{2} / 2}
$$

However, one useful way to proceed is to recognize that this latter sum can be rewritten as

$$
\left.\frac{1}{(2 \pi i)^{n}} \frac{\partial^{n}}{\partial z_{\ell}^{n}} \vartheta_{\ell}\left(z_{\ell} \mid \tau\right)\right|_{z_{\ell=0}}
$$

where the generalized $\theta_{\ell}\left(z_{\ell} \mid \tau\right)$ function is defined as

$$
\vartheta_{\ell}\left(z_{\ell} \mid \tau\right) \equiv \sum_{Q_{\ell} \in \mathbb{Z}+\alpha_{\ell}} e^{2 \pi i\left(\beta_{\ell}+z_{\ell}\right) Q_{\ell}} q^{Q_{\ell}^{2} / 2}
$$

Indeed, we see that $\vartheta_{\ell}(\tau)$ is nothing but $\vartheta_{\ell}\left(z_{\ell} \mid \tau\right)$ evaluated at $z_{\ell}=0$. However, for arbitrary $z$, these generalized $\vartheta(z \mid \tau)$ functions have the schematic modular-transformation properties

$\vartheta_{\ell}\left(z \mid \frac{a \tau+b}{c \tau+d}\right) \sim(c \tau+d)^{1 / 2} e^{\pi i c(c \tau+d) z^{2}} \vartheta_{\ell}((c \tau+d) z \mid \tau)$.

It then follows that

$$
\left.\left.\vartheta\left(z \mid \frac{a \tau+b}{c \tau+d}\right)\right|_{z=0} \sim(c \tau+d)^{1 / 2} \vartheta(z \mid \tau)\right|_{z=0},
$$

and likewise

$$
\left.\left.\frac{\partial}{\partial z} \vartheta\left(z \mid \frac{a \tau+b}{c \tau+d}\right)\right|_{z=0} \sim(c \tau+d)^{3 / 2} \frac{\partial}{\partial z} \vartheta(z \mid \tau)\right|_{z=0} .
$$

This indicates that while the function $\left.\vartheta(z \mid \tau)\right|_{z=0}$ transforms covariantly with modular weight $1 / 2$, its first derivative $\left.[\partial \vartheta(z \mid \tau) / \partial z]\right|_{z=0}$ transforms covariantly with modular weight $3 / 2$.

At first glance, one might expect this pattern to continue, with the second derivative $\left.\left[\partial^{2} \vartheta(z \mid \tau) / d z^{2}\right]\right|_{z=0}$ transforming covariantly with modular weight $5 / 2$. However, this is not what happens. Instead, from Eq. (2.37) we find

$$
\begin{aligned}
\left.\frac{\partial^{2}}{\partial z^{2}} \vartheta\left(z \mid \frac{a \tau+b}{c \tau+d}\right)\right|_{z=0} \sim & \left.(c \tau+d)^{5 / 2} \frac{\partial}{\partial z} \vartheta(z \mid \tau)\right|_{z=0} \\
& +2 \pi i c(c \tau+d)^{3 / 2} \vartheta(\tau) .
\end{aligned}
$$

While the term on the first line is the expected result, the term on the second line represents a modular anomaly which destroys the modular covariance of the second derivative.

Since modular covariance must be preserved, we must perform a modular completion. In this simple case, this means that we must replace $\partial^{2} / \partial z^{2}$ with a modularcovariant second derivative $D_{z}^{2}$ such that $D_{z}^{2}$ not only contains $\partial^{2} / \partial z^{2}$ but also has the property that $\left.D_{z}^{2} \theta(z \mid \tau)\right|_{z=0}$ transforms covariantly with weight $5 / 2$. It is straightforward to show that the only such modular-covariant derivative is

$$
D_{z}^{2} \equiv \frac{\partial^{2}}{\partial z^{2}}+\frac{\pi}{\tau_{2}}
$$

and with this definition one indeed finds

$$
\left.\left.\left\{\left[D_{z}^{2} \vartheta(z \mid \tau)\right]_{\tau \rightarrow \frac{a c+b}{c \tau+d}}\right\}\right|_{z=0} \sim(c \tau+d)^{5 / 2} D_{z}^{2} \vartheta(z \mid \tau)\right|_{z=0},
$$

thereby continuing the pattern set by Eqs. (2.38) and (2.39). It turns out that this modular-covariant second $z$-derivative is equivalent to the modular-covariant $\tau$-derivative

$$
D_{\tau} \equiv \frac{\partial}{\partial \tau}-\frac{i k}{2 \tau_{2}}
$$

which preserves the modular covariance of any modular function of weight $k$. Indeed, our $\vartheta(z \mid \tau)$ functions have $k=1 / 2$ and satisfy the heat equation $\partial^{2} \vartheta(z \mid \tau) / \partial z^{2}=$ $4 \pi i \partial \vartheta(z \mid \tau) / \partial \tau$. In this sense, the $z$-derivative serves as a "square root" of the $\tau$-derivative and gives us a precise means of extracting the individual charge insertions (rather than their squares). In this connection, we emphasize that there is a tight correspondence between the Higgs field and the z-parameter. Specifically, when we deform a theory through a continuous change in the value of the Higgs VEV, its partition function deforms through a corresponding continuous change in the $z$ parameter.

In principle we could continue to examine higher $z$-derivatives (all of which will also suffer from modular anomalies), but the results we have thus far will be sufficient for our purposes. Recalling the equivalence between the expressions in Eqs. (2.34) and (2.35), we thus see that the insertion of a single power of any given $Q_{\ell}$ does not disturb the modular covariance of the corresponding holomorphic (or antiholomorphic) factor in the partition-function trace, but the insertion of a quadratic term $Q_{\ell}^{2}$ along the $\ell$ th lattice direction does not lead to a modular-covariant result and must, according to Eq. (2.41), be replaced by the modularcovariant insertion $Q_{\ell}^{2}-1 /\left(4 \pi \tau_{2}\right)$. Thus, our rules for modular completion through second order in charge-vector components are given by

$$
\left\{\begin{array}{l}
\mathbf{Q}_{\ell} \rightarrow \mathbf{Q}_{\ell}, \\
\mathbf{Q}_{\ell} \mathbf{Q}_{\ell^{\prime}} \rightarrow \mathbf{Q}_{\ell} \mathbf{Q}_{\ell^{\prime}}-\frac{1}{4 \pi \tau_{2}} \delta_{\ell, \ell^{\prime}}
\end{array}\right.
$$

These general results hold for all lattice directions $\left(\ell, \ell^{\prime}\right)$ regardless of whether they correspond to left- or rightmoving lattice components. Such modular completions have also arisen in other contexts, such as within string-theoretic threshold corrections [4-6]. 
With these modular-completion rules in hand, we can now investigate the modular completion of the expression for $\mathcal{X}$ in Eq. (2.33). It is simplest to begin by focusing on the quartic terms, i.e., the terms in the top line of Eq. (2.33). Given the identity just below Eq. (2.32), these terms are proportional to $\left(\mathbf{Q}_{L}^{t} \tilde{\mathbf{Q}}_{h}\right)^{2}$. With $Q_{L \ell}$ denoting the $\ell$ th component of $\mathbf{Q}_{L}$, etc., we find

$$
\begin{aligned}
\left(\mathbf{Q}_{L}^{t} \tilde{\mathbf{Q}}_{h}\right)^{2}= & \left(\sum_{\ell=1}^{22} \sum_{m=1}^{10} Q_{L \ell}\left(\mathcal{T}_{12}\right)_{\ell m} Q_{R m}\right)^{2} \\
= & \sum_{\ell, \ell^{\prime}=1}^{22} \sum_{m, m^{\prime}=1}^{10}\left(\mathcal{T}_{12}\right)_{\ell m}\left(\mathcal{T}_{12}\right)_{\ell^{\prime} m^{\prime}} \\
& \times Q_{R m} Q_{R m^{\prime}} Q_{L \ell} Q_{L \ell^{\prime}} .
\end{aligned}
$$

Following the rules in Eq. (2.44), we can readily obtain the modular completion of this expression by replacing the final line in Eq. (2.45) with

$$
\left(Q_{R m} Q_{R m^{\prime}}-\frac{1}{4 \pi \tau_{2}} \delta_{m m^{\prime}}\right)\left(Q_{L \ell} Q_{L \ell^{\prime}}-\frac{1}{4 \pi \tau_{2}} \delta_{\ell \ell^{\prime}}\right) .
$$

Substituting Eq. (2.46) into Eq. (2.45) and recalling that $\mathcal{T}_{12}^{t}=-\mathcal{T}_{21}$, we thus find that the modular completion of the quartic term $\left(\mathbf{Q}_{L}^{t} \tilde{\mathbf{Q}}_{h}\right)^{2}$ within $\mathcal{X}$ is given by

$$
\left(\mathbf{Q}_{L}^{t} \tilde{\mathbf{Q}}_{h}\right)^{2}-\frac{1}{4 \pi \tau_{2}}\left(\mathbf{Q}_{h}^{2}+\tilde{\mathbf{Q}}_{h}^{2}\right)+\frac{\xi}{\left(4 \pi \tau_{2}\right)^{2}}
$$

where

$$
\begin{aligned}
\xi & \equiv \operatorname{Tr}\left(\mathcal{T}_{12}^{t} \mathcal{T}_{12}\right)=\operatorname{Tr}\left(\mathcal{T}_{21}^{t} \mathcal{T}_{21}\right) \\
& =-\operatorname{Tr}\left(\mathcal{T}_{12} \mathcal{T}_{21}\right)=-\operatorname{Tr}\left(\mathcal{T}_{21} \mathcal{T}_{12}\right)
\end{aligned}
$$

Remarkably, the quadratic terms $\mathbf{Q}_{h}^{2}+\tilde{\mathbf{Q}}_{h}^{2}$ that are generated within Eq. (2.47) already appear on the second line of Eq. (2.33). In other words, even if we had not already known of these quadratic terms, we could have deduced their existence through the modular completion of our quartic terms. Conversely, we could have generated the quartic terms through a modular completion of these quadratic terms-i.e., each set of terms provides the modular completion of the other. Thus, the only remaining terms within Eq. (2.33) that might require modular completion are the final quadratic terms on the second line of Eq. (2.33), namely $\tilde{\mathbf{Q}}_{j}^{t} \mathbf{Q}_{h}+\mathbf{Q}_{j}^{t} \tilde{\mathbf{Q}}_{h}$. However, $\mathbf{Q}_{h}$ and $\mathbf{Q}_{j}$ involve only left-moving components of the lattice while $\tilde{\mathbf{Q}}_{h}$ and $\tilde{\mathbf{Q}}_{j}$ involve only right-moving components. Thus $\tilde{\mathbf{Q}}_{j}^{t} \mathbf{Q}_{h}+\mathbf{Q}_{j}^{t} \tilde{\mathbf{Q}}_{h}$ is already modular complete. Putting all the pieces together, we therefore find that the total expression for $\mathcal{X}$ in Eq. (2.33) has a simple (and in fact universal) modular completion:

$$
\mathcal{X} \rightarrow \mathcal{X}+\frac{\xi}{4 \pi^{2}}
$$

Indeed, this sole remaining extra term generated by the modular completion stems from the final term in Eq. (2.47). It is noteworthy that this extra term is entirely independent of the charge vectors. This is consistent with our expectation that such additional terms represent the contributions from the deformations of the moduli fields under Higgs fluctuations - deformations which act in a universal (and hence $Q$-independent) manner.

Some remarks are in order regarding the uniqueness of the completion in Eq. (2.49). In particular, at first glance one might wonder how the modular completion of the quadratic terms $\tilde{\mathbf{Q}}_{j}^{t} \mathbf{Q}_{h}+\mathbf{Q}_{j}^{t} \tilde{\mathbf{Q}}_{h}$ could uniquely determine the quartic terms in $\mathcal{X}$, given that the modular-completion rules within Eq. (2.44) only seem to generate extra terms which are of lower powers in charge-vector components. However, the important point is that the rules in Eq. (2.44) only ensure the modular covariance of the individual (anti) holomorphic components of the partition-function trace. In particular, these rules do not, in and of themselves, ensure that we continue to satisfy the additional constraint in Eq. (2.12) that arises when stitching these holomorphic and antiholomorphic components together as in Eq. (2.11). However, given that $\mathbf{Q}_{h}^{2}$ increases the modular weight of the holomorphic component by two units without increasing the modular weight of the antiholomorphic component, and given that $\tilde{\mathbf{Q}}_{h}^{2}$ does the opposite, the only way to properly modular-complete their sum is by "completing the square" and realizing these terms as the off-diagonal terms that are generated through a factorized modular completion as in Eq. (2.46). This then compels the introduction of the appropriate quartic diagonal terms, as seen above.

In this connection, it is also important to note that modular completion involves more than simply demanding that our final result be modular invariant. After all, we have seen in Eq. (2.49) that the modular completion of $\mathcal{X}$ involves the addition of a pure number, i.e., the addition of a quantity which is intrinsically modular-invariant on its own (or more precisely, a quantity whose insertion into the partition-function summand automatically preserves the modular invariance of the original partition function). However, as we have stated above, modular completion ensures more than the mere modular invariance of our final result - it also ensures that this result is obtainable through a modular-covariant sequence of calculational operations. As we have seen, the extra additive constant that forms the modular completion of $\mathcal{X}$ in Eq. (2.49) is crucial in allowing us to "complete the square" and thereby cast our results into the factorized form of Eq. (2.46) - a form which itself emerged as a consequence of our underlying modularcovariant $z$-derivatives $D_{z}^{2}$. As such, the constant appearing in Eq. (2.49) is an intrinsic part of our resulting expression for $m_{\phi}^{2}$. 


\section{F. Classical stability condition}

Thus far, we have focused on deriving an expression for the Higgs mass, as defined in Eq. (2.22). However, our results presuppose that we are discussing a classically stable particle. In other words, while we are identifying the mass with the second $\phi$-derivative of the classical potential, we are implicitly assuming that the first $\phi$-derivative vanishes so that we are sitting at a minimum of the Higgs potential. Thus, there is an extra condition that we need to impose, namely

$$
\left.\frac{d \Lambda(\phi)}{d \phi}\right|_{\phi=0}=0
$$

This condition must be satisfied for the particular vacuum state within which our Higgs-mass calculation has been performed.

It is straightforward to determine the ramifications of this condition. Proceeding exactly as above, we find in analogy with Eq. (2.27) that $\partial \mathcal{Z} /\left.\partial \phi\right|_{\phi=0}$ corresponds to an insertion given by $\left.Y\right|_{\phi=0}=\mathcal{Y} / \mathcal{M}$, where

$$
\mathcal{Y} \sim \tau_{2}\left(\mathbf{Q}_{R}^{t} \mathbf{Q}_{h}-\mathbf{Q}_{L}^{t} \tilde{\mathbf{Q}}_{h}\right) \sim \tau_{2}\left(\mathbf{Q}_{R}^{t} \mathcal{T}_{21} \mathbf{Q}_{L}\right) .
$$

Given this result, there are a priori three distinct ways in which the condition in Eq. (2.50) can be satisfied within a given string vacuum. First, $\mathcal{Y}$ might vanish for each state in the corresponding string spectrum. Second, $\mathcal{Y}$ might not vanish for each state in the string spectrum but may vanish in the sum over the string states (most likely in a pairwise fashion between chiral and antichiral states with opposite charge vectors). However, there is also a third possibility: the entire partition-function trace may be nonzero, even with the $\mathcal{Y}$ insertion, but nevertheless vanish when integrated over the fundamental domain of the modular group, as in Eq. (2.23). In general, very few mathematical examples are known of situations in which this latter phenomenon occurs [7-9], although the fact that this would involve an integrand with vanishing modular weight offers unique possibilities.

Two further comments regarding this condition are in order. First, it is easy to verify that this condition respects modular invariance, as it must. Indeed, the quantity $\mathcal{Y}$, as defined above, is already modular complete. At first glance, this might seem surprising, given that the quartic terms within $\mathcal{X}$ are nothing but the square of $\mathcal{Y}$, and we have already seen that these quartic terms are not modular complete by themselves. However, it is the squaring of $\mathcal{Y}$ that introduces the higher powers of charge-vector components which in turn induce the modular anomaly. Second, if $\mathcal{Y}$ vanishes when summed over all of the string states, then it might be tempting to hope that the quartic terms within $\mathcal{X}$ also vanish when summed over the string states. Unfortunately, this hope is not generally realized, since important sign information is lost when these quantities are squared. Of course, if $\mathcal{Y}$ vanishes for each individual state in the string spectrum, then the quartic terms within $\mathcal{X}$ will also evaluate to zero in any calculation of the corresponding Higgs mass. This would then simplify the explicit evaluation of $\mathcal{X}$ for such a string vacuum.

\section{G. A relation between the Higgs mass and the cosmological constant}

Let us now collect our results for the Higgs mass. For notational simplicity we define

$$
\langle A\rangle \equiv \int_{\mathcal{F}} \frac{d^{2} \tau}{\tau_{2}^{2}} \frac{\tau_{2}^{-1}}{\bar{\eta}^{12} \eta^{24}} \sum_{\mathbf{Q}_{L}, \mathbf{Q}_{R}}(-1)^{F} A \bar{q}^{\mathbf{Q}_{R}^{2} / 2} q^{\mathbf{Q}_{L}^{2} / 2}
$$

where the charge vectors $\left\{\mathbf{Q}_{L}, \mathbf{Q}_{R}\right\}$ in the sum over states are henceforth understood as unperturbed (i.e., with $\phi=0$ ) and thus correspond directly to the charges that arise at the minimum of the Higgs potential. Our results then together imply that

$$
m_{\phi}^{2}=-\frac{\mathcal{M}^{2}}{2}\langle\mathcal{X}\rangle-\frac{\mathcal{M}^{2}}{2} \frac{\xi}{4 \pi^{2}}\langle\mathbf{1}\rangle
$$

where $\mathcal{X}$ is given in Eq. (2.33). As indicated above, these results implicitly assume that $\langle\mathcal{Y}\rangle=0$, where $\mathcal{Y}$ is defined in Eq. (2.51). However, we immediately recognize that the quantity $\langle\mathbf{1}\rangle$ within Eq. (2.53) is nothing other than the oneloop zero-point function (cosmological constant) $\Lambda$. More precisely, we may identify $\Lambda$ as $\left.\Lambda(\phi)\right|_{\phi=0}$ [where $\Lambda(\phi)$ is given in Eq. (2.23)], or equivalently

$$
\Lambda=-\frac{\mathcal{M}^{4}}{2}\langle\mathbf{1}\rangle \text {. }
$$

We thus obtain the relation

$$
m_{\phi}^{2}=\frac{\xi}{4 \pi^{2}} \frac{\Lambda}{\mathcal{M}^{2}}-\frac{\mathcal{M}^{2}}{2}\langle\mathcal{X}\rangle .
$$

Indeed, retracing our steps in arbitrary spacetime dimension $D$, we obtain the analogous relation

$$
m_{\phi}^{2}=\frac{\xi}{4 \pi^{2}} \frac{\Lambda}{\mathcal{M}^{D-2}}-\frac{\mathcal{M}^{2}}{2}\langle\mathcal{X}\rangle
$$

where the cosmological constant $\Lambda$ now has mass dimension $D$.

Remarkably, this is a general relation between the Higgs mass and the one-loop cosmological constant. Because this relation rests on nothing but modular invariance, it holds generally for any perturbative closed string in any arbitrary spacetime dimension $D$. The cosmological-constant term in Eq. (2.55) is universal, emerging as the result of a modular anomaly that required a modular completion, or equivalently as the result of a universal shift in the moduli. 
By contrast, the second term depends on the particular charges that are inserted into the partition-function trace.

For weakly coupled heterotic strings, we can push this relation one step further. In such theories the string scale $M_{s} \equiv 2 \pi \mathcal{M}$ and Planck scale $M_{p}$ are connected through the relation $M_{s}=g_{s} M_{P}$ where $g_{s}$ is the string coupling whose value is set by the vacuum expectation value of the dilaton. Depending on the particular string model, $g_{s}$ in turn sets the values of the individual gauge couplings. Likewise, the canonically normalized scalar field $\phi$ is $\hat{\phi} \equiv \phi / g_{s}$. We thus find that our relation in Eq. (2.55) equivalently takes the form

$$
m_{\hat{\phi}}^{2}=\frac{\xi}{M_{P}^{2}} \Lambda-\frac{g_{s}^{2} \mathcal{M}^{2}}{2}\langle\mathcal{X}\rangle .
$$

In quantum field theory, we would not expect to find a relation between a Higgs mass and a cosmological constant. Indeed, quantum field theories do not involve gravity and are thus insensitive to the absolute zero of energy. Even worse, in quantum field theory, the one-loop zero-point function is badly divergent. String theory, by contrast, not only unifies gauge theories with gravity but also yields a finite $\Lambda$ (the latter occurring as yet another by-product of modular invariance). Thus, it is only within a string context that such a relation could ever arise, and indeed Eqs. (2.55) and (2.57) are precisely the relations that arise for all weakly coupled four-dimensional heterotic strings. We expect that this is but the tip of the iceberg, and that other modular-invariant string constructions lead to similar results. It is intriguing that such relations join together precisely the two quantities $\left(m_{\phi}\right.$ and $\left.\Lambda\right)$ whose values lie at the heart of the two most pressing hierarchy problems in modern physics.

\section{REGULATING THE HIGGS MASS: FROM AMPLITUDES TO SUPERTRACES}

In Eq. (2.55) we obtained a result in which the Higgs mass, via the definition in Eq. (2.52), is expressed in terms of certain one-loop string amplitudes consisting of modular integrals of various traces over the entire string spectrum. As discussed below Eq. (2.5), these traces include the contributions of not only physical (i.e., level-matched) string states with $M_{L}^{2}=M_{R}^{2}$, but also unphysical (i.e., non-levelmatched) string states with $M_{L}^{2} \neq M_{R}^{2}$. This distinction between physical and unphysical string states is important because only the physical string states can serve as bona fide in- and out-states. By contrast, the unphysical states are intrinsically stringy and have no field-theoretic analogs.

We now wish to push our calculation several steps further. In particular, there are three aspects to our result in Eq. (2.55) which we will need to understand in order to allow us to make contact with traditional quantum-fieldtheoretic expectations. The first concerns the fact that while the one-loop vacuum energy $\Lambda$ which appears in these results is finite for all tachyon-free string models - even without spacetime supersymmetry-the remaining amplitude $\langle\mathcal{X}\rangle$ which appears in these expressions is generically divergent. Note that this is not in conflict with stringtheoretic expectations; in particular, as we shall discuss in Sec. IVA, string theory generally softens various fieldtheoretic divergences but need not remove them entirely. Thus, our expression for the Higgs mass is formally divergent and requires some sort of regulator in order to extract finite results. Second, while these results are expressed in terms of sums over the entire string spectrum, we would like to be able to express the Higgs mass directly in terms of supertraces over only the physical string states-i.e., the states with direct field-theoretic analogs. This will ultimately allow us to express the Higgs mass in a form that might be recognizable within ordinary quantum field theory, and thereby extract an EFT description of the Higgs mass in which our Higgs mass experiences an effective renormalization-group "running." This will also allow us to extract a stringy effective potential for the Higgs field. Finally, as a by-product, we would also like to implicitly perform the stringy modular integrations inherent in Eq. (2.52).

As it turns out, these three issues are intimately related. However, appreciating these connections requires a deeper understanding of the properties of the modular functions on which which our Higgs-mass calculations rest. In this section, we shall therefore outline the mathematical procedures which will enable us to address all three of our goals. Many of these methods originated in the classic mathematics papers of Rankin [10,11] and Selberg [12] from the late 1930s, and were later extended in an important way by Zagier [13] in the early 1980s. Some of the Rankin-Selberg results also later independently found their way into the string literature in various forms [14-16], and have occasionally been studied and further developed (see, e.g., Refs. [17-25]). Our purpose in recounting these results here is not only to pull them all together and explain their logical connections in relatively nontechnical terms, but also to extend them in certain directions which will be important for our work in Sec. IV. This conceptual and mathematical groundwork will thus form the underpinning for our further analysis of the Higgs mass in Sec. IV.

\section{A. The Rankin-Selberg technique}

We are interested in modular integrals such as those in Eq. (2.52) which generically take the form

$$
I \equiv \int_{\mathcal{F}} \frac{d^{2} \tau}{\tau_{2}^{2}} F(\tau, \bar{\tau})
$$

where $\mathcal{F}$ is the modular-group fundamental domain given in Eq. (2.24), where $d \tau_{1} d \tau_{2} / \tau_{2}^{2}$ is the modular-covariant 
integration measure (with $\tau \equiv \tau_{1}+i \tau_{2}, \tau_{i} \in \mathbb{R}$ ), and where the integrand $F$ is modular invariant. In general the integrands $F$ take the form

$$
F \equiv \tau_{2}^{k} \sum_{m, n} a_{m n} \bar{q}^{m} q^{n}
$$

where $q \equiv e^{2 \pi i \tau}$ and where $k$ is the modular weight of the holomorphic and antiholomorphic modular functions whose products contribute to $F$. Note that integrands of this form include those in Eq. (2.1): we simply powerexpand the $\eta$-function denominators and absorb these powers into $m$ and $n$. Thus, with string integrands written as in Eq. (3.2) we can now directly identify $m=\alpha^{\prime} M_{R}^{2} / 4$ and $n=\alpha^{\prime} M_{L}^{2} / 4$. The quantity $a_{m n}$ then tallies the number of bosonic minus fermionic string degrees of freedom contributing to each $\left(M_{R}^{2}, M_{L}^{2}\right)$ term.

Invariance under $\tau \rightarrow \tau+1$ guarantees that every term within $F$ has $m-n \in \mathbb{Z}$. The $m=n$ terms represent the contributions from physical string states with spacetime masses $\alpha^{\prime} M^{2}=2(m+n)=4 n$, while the $m \neq n$ terms represent the contributions from off-shell (i.e., unphysical) string states. Within the $\tau_{2} \geq 1$ integration subregion within $\mathcal{F}$, the $m \neq n$ terms make no contribution to the integral $I$ because these contributions are eliminated when we perform the $\int_{-1 / 2}^{1 / 2} d \tau_{1}$ integral. [Indeed, within this subregion of $\mathcal{F}$ expressions such as Eq. (3.1) come with an implicit instruction that we are to perform the $\tau_{1}$ integration prior to performing the $\tau_{2}$ integration.] However, the full integral $I$ does receive $m \neq n$ contributions from the $\tau_{2}<1$ subregion within $\mathcal{F}$. Thus, in general, both physical and unphysical string states contribute to amplitudes such as $I$.

Our goal is to express $I$ in terms of contributions from the physical string states alone. Clearly this could be done if we could somehow transform the region of integration within $I$ from the fundamental domain $\mathcal{F}$ to the positive half-strip

$$
\mathcal{S} \equiv\left\{\tau:-\frac{1}{2}<\tau_{1} \leq \frac{1}{2}, \tau_{2}>0\right\}
$$

for we would then have

$$
\int_{\mathcal{S}} \frac{d^{2} \tau}{\tau_{2}^{2}} F(\tau, \bar{\tau})=\int_{0}^{\infty} \frac{d \tau_{2}}{\tau_{2}^{2}} g\left(\tau_{2}\right)
$$

where $g\left(\tau_{2}\right)$ is our desired trace over only the physical string states:

$$
g\left(\tau_{2}\right)=\int_{-1 / 2}^{1 / 2} d \tau_{1} F(\tau, \bar{\tau})=\tau_{2}^{k} \sum_{n} a_{n n} e^{-4 \pi \tau_{2} n} .
$$

Fortunately, there exists a well-known method for "unfolding" $\mathcal{F}$ into $\mathcal{S}$. While $\mathcal{F}$ is the fundamental domain of the modular group $\Gamma$ generated by both $\tau \rightarrow-1 / \tau$ and $\tau \rightarrow \tau+1$, the strip $\mathcal{S}$ is the fundamental domain of the modular subgroup $\Gamma_{\infty}$ generated solely by $\tau \rightarrow \tau+1$. (Indeed, this is the subgroup that preserves the cusp at $\tau=i \infty$.) Thus the strip $\mathcal{S}$ can be realized as the sum of the images of $\mathcal{F}$ transformed under all modular transformations $\gamma$ (including the identity) in the coset $\Gamma_{\infty} \backslash \Gamma$ :

$$
\mathcal{S}=\underset{\gamma \in \Gamma_{\infty} \backslash \Gamma}{\cup} \gamma \cdot \mathcal{F}
$$

It then follows for any integrand $\tilde{F}(\tau, \bar{\tau})$ that

$$
\int_{\mathcal{S}} \frac{d^{2} \tau}{\tau_{2}^{2}} \tilde{F}(\tau, \bar{\tau})=\int_{\mathcal{F}} \frac{d^{2} \tau}{\tau_{2}^{2}} \sum_{\gamma \in \Gamma_{\infty} \backslash \Gamma} \tilde{F}_{\gamma}(\tau, \bar{\tau}),
$$

where $\tilde{F}_{\gamma}(\tau, \bar{\tau})$ is the $\gamma$-transform of $\tilde{F}(\tau, \bar{\tau})$. Moreover, if $\tilde{F}(\tau, \bar{\tau})$ is invariant under $\tau \rightarrow \tau+1$, then the total integrand on the right side of Eq. (3.7) is modular invariant.

At this stage, armed with the result in Eq. (3.7), we see that we are halfway toward our goal. However, two fundamental problems remain. First, while choosing $\tilde{F}$ as our original integrand $F$ would allow us to express the left side of Eq. (3.7) directly in terms of the desired trace in Eq. (3.5), our need to relate this to the original integral $I$ in Eq. (3.1) would instead seem to require choosing $\tilde{F}$ such that $F=\sum_{\gamma \in \Gamma_{\infty} \backslash \Gamma} \tilde{F}_{\gamma}$. Second, the manipulations underlying Eq. (3.7), such as the exchanging of sums and regions of integration, implicitly assumed that the integrand on the right side of Eq. (3.7) converges sufficiently rapidly as $\tau_{2} \rightarrow \infty$ [or equivalently that the integrand on the left side of Eq. (3.7) converges sufficiently rapidly as $\tau_{2} \rightarrow 0$ ] so that all relevant integrals are absolutely convergent. However, this is generally not the case for the physical situations that will interest us.

It turns out that these problems together motivate a unique choice for $\tilde{F}$. Note that $g\left(\tau_{2}\right)$ generally has a form resembling that in Eq. (3.5), consisting of an infinite sum multiplied by a power of $\tau_{2}$. As $\tau_{2} \rightarrow 0$, the successive terms in this sum are less and less suppressed by the exponential factor $e^{-4 \pi n \tau_{2}}$. We therefore expect the infinite sum within $g\left(\tau_{2}\right)$ to experience an increased tendency to diverge as $\tau_{2} \rightarrow 0$. Let us assume for the moment that the divergence of this infinite sum grows no faster than some inverse power of $\tau_{2}$ as $\tau_{2} \rightarrow 0$. In this case, the divergence of the sum within $g\left(\tau_{2}\right)$ will cause $g\left(\tau_{2}\right)$ itself to diverge as $\tau_{2} \rightarrow 0$ unless $g\left(\tau_{2}\right)$ also includes a prefactor consisting of sufficiently many powers of $\tau_{2}$ to hold the divergence of the sum in check. We can therefore regulate our calculation by introducing sufficiently many extra powers of $\tau_{2}$ into $g\left(\tau_{2}\right)$. In other words, in such cases we shall take

$$
\tilde{F}(\tau, \bar{\tau})=\tau_{2}^{s} F(\tau, \bar{\tau})
$$

where $s$ is chosen sufficiently large (typically requiring $s>1$ ) so as to guarantee convergence. Indeed, since the 
number of powers of $\tau_{2}$ within $g\left(\tau_{2}\right)$ is generally correlated in string theory with the number of uncompactified spacetime dimensions, we may view this insertion of extra powers of $\tau_{2}$ as a stringy version of dimensional regularization, taking $D \rightarrow D_{\text {eff }} \equiv D-2 s$. However, since our original integrand $F(\tau, \bar{\tau})$ is presumed modular invariant, the choice in Eq. (3.8) in turn implies that the integrand on the right side of Eq. (3.7) must be taken as

$$
\sum_{\gamma \in \Gamma_{\infty} \backslash \Gamma}(\operatorname{Im} \gamma \cdot \tau)^{s} F_{\gamma}(\tau, \bar{\tau})=E(\tau, \bar{\tau}, s) F(\tau, \bar{\tau})
$$

where $E(\tau, \bar{\tau}, s)$ is the nonholomorphic Eisenstein series, often simply denoted $E(\tau, s)$ and defined by

$E(\tau, s) \equiv \sum_{\gamma \in \Gamma_{\infty} \backslash \Gamma}[\operatorname{Im}(\gamma \cdot \tau)]^{s}=\frac{1}{2} \sum_{(c, d)=1} \frac{\tau_{2}^{s}}{|c \tau+d|^{2 s}}$

with the second sum in Eq. (3.10) restricted to integer, relatively prime values of $c, d$. Thus, with these choices, we now have

$$
\int_{\mathcal{F}} \frac{d^{2} \tau}{\tau_{2}^{2}} E(\tau, s) F(\tau, \bar{\tau})=\int_{0}^{\infty} d \tau_{2} \tau_{2}^{s-2} g\left(\tau_{2}\right)
$$

where the expression on the right side depends on only the physical string states.

The Eisenstein series $E(\tau, s)$ has a number of important properties. It is convergent for all $s>1$, but can be analytically continued to all values of $s$. It is not only modular invariant (consistent with $\mathcal{F}$ as the corresponding region of integration), but its insertion on the left side of Eq. (3.11) relative to our original starting point in Eq. (3.1) softens the divergence as $\tau_{2} \rightarrow \infty$, as required. Most importantly for our purposes, however, this function has a simple pole at $s=1$, with a $\tau$-independent residue $3 / \pi$. The fact that this residue is $\tau$-independent means that we can formally extract our original integral $I$ in Eq. (3.1) by taking the $s=1$ residue of both sides of Eq. (3.11):

$$
I=\frac{\pi}{3} \operatorname{Res}_{s=1} \int_{0}^{\infty} d \tau_{2} \tau_{2}^{s-2} g\left(\tau_{2}\right)
$$

We have therefore succeeded in expressing our original modular integral $I$ in terms of only the contributions from the physical states. The result in Eq. (3.12) was originally obtained by Rankin and Selberg in 1939 (see, e.g., Refs. [10-12]), and has proven useful for a number of applications in both physics and pure mathematics.

At this stage, three important comments are in order. First, it may seem that the result in Eq. (3.12) implies that the unphysical states ultimately make no contributions to the amplitude $I$. However, this is untrue: the result in Eq. (3.12) was derived under the supposition that our original integrand $F(\tau, \bar{\tau})$ is modular invariant, and this modular invariance depends crucially on the existence of both physical and unphysical states in the full string spectrum. For example, through the requirement of modular invariance, the distribution of unphysical states in the string spectrum has a profound effect $[17,18]$ on the values of the physical-state degeneracies $\left\{a_{n n}\right\}$ which appear in Eq. (3.5).

As our second comment, we point out that the above results can be reformulated in a manner which eliminates the $\tau_{2}$ integration completely and which depends directly on the integrand $g\left(\tau_{2}\right)$. To see this, we note if we define $I(s)$ as the term on the left side of Eq. (3.11), then the relation in Eq. (3.11) simply states that $I(s)$ is nothing but the Mellin transform of $g\left(\tau_{2}\right) / \tau_{2}$. One can therefore use the inverse Mellin transform to write $g\left(\tau_{2}\right) / \tau_{2}$ directly in terms of $I(s)$. While such an inverse relation is useful in many contexts, for our purposes it will be sufficient to note that such an inverse relation implies a direct connection between the poles of $I(s)$ and the asymptotic behavior of $g\left(\tau_{2}\right)$ as $\tau_{2} \rightarrow 0$. Specifically, one finds a correlation

$$
\begin{gathered}
\text { poles of } I(s) \text { at } s=s_{n} \text { with residues } c_{n} \\
\quad \Rightarrow g\left(\tau_{2}\right) \sim \sum_{n} c_{n} \tau_{2}^{1-s_{n}} \quad \text { as } \tau_{2} \rightarrow 0 .
\end{gathered}
$$

As we have seen, $I(s)$ has a single pole along the real axis at $s=1$, with residue $3 I / \pi$. However, $I(s)$ also has an infinite number of poles at locations $s_{n}=\rho_{n} / 2$, where $\rho_{n}$ are the nontrivial zeros of the Riemann $\zeta$ function $\zeta(s)$. According to the Riemann hypothesis, these zeros all have the form $\rho_{n}=\frac{1}{2} \pm i \gamma_{n}$ where $\gamma_{n} \in \mathbb{R}$. The fact that $\operatorname{Re}\left(s_{n}\right)<1$ for all of these additional poles of $I(s)$ then implies that the amplitude $I$ dominates the leading behavior of $g\left(\tau_{2}\right)$ as $\tau_{2} \rightarrow 0$, allowing us to write $[13,16]$

$$
I=\frac{\pi}{3} \lim _{\tau_{2} \rightarrow 0} g\left(\tau_{2}\right)
$$

Of course, from Eq. (3.13) we see that the $\tau_{2} \rightarrow 0$ limit of $g\left(\tau_{2}\right)$ also contains subleading oscillatory terms [13] corresponding to the nontrivial zeros of the $\zeta$ function. This suggests, through Eq. (3.5), that the $a_{n n}$ coefficients tend to oscillate in sign as $n \rightarrow \infty$. This oscillating sign is in fact a consequence of the so-called "misaligned supersymmetry" [17-19] which is a generic property of all tachyon-free nonsupersymmetric string models - a property whose existence is a direct consequence of modular invariance in general situations where $I$ is finite and $F(\tau, \bar{\tau}) \neq 0$.

Our final comment, however, is perhaps the most crucial. As we have seen, the results in Eqs. (3.12) and (3.14) were derived under the assumption, as stated within the above derivation, that the infinite sum within the definition of $g\left(\tau_{2}\right)$ in Eq. (3.5) diverges no more rapidly than some inverse power of $\tau_{2}$ as $\tau_{2} \rightarrow 0$. This requirement was needed so that the introduction of sufficiently many $\tau_{2}$ prefactors could suppress this divergence and render a finite result. Undoing the modular transformations involved in Eq. (3.7), we see that this is equivalent to demanding that 
our original integrand $F(\tau, \bar{\tau})$ either fall, remain constant, or grow less rapidly than $\tau_{2}$ as $\tau_{2} \rightarrow \infty$. Indeed, these are the conditions under which the Rankin-Selberg analysis is valid. Not surprisingly, these are also the conditions under which any integrand $F$ lacking terms with $m=n<0$ will produce a finite value for $I$.

\section{B. Regulating divergences}

The techniques discussed in Sec. III A are completely adequate for situations in which the original amplitude $I$ is finite, with an integrand $F(\tau, \bar{\tau})$ remaining finite or diverging less rapidly than $\tau_{2}$ as $\tau_{2} \rightarrow \infty$. However, many physical situations of interest (including those we shall ultimately need to consider in this paper) lead to integrands $F(\tau, \bar{\tau})$ which diverge more rapidly than this as $\tau_{2} \rightarrow \infty$. As a result, the corresponding integral $I$ formally diverges and must be regulated.

In this section we shall discuss three different methods of regulating such amplitudes. These methods are appropriate for cases-such as we shall ultimately face-in which the integrand experiences a power-law divergence $\sim \tau_{2}^{p}$ with $p \geq 1$ as $\tau_{2} \rightarrow \infty$. As we shall see, these regulators each have different strengths and weaknesses, and thus it will prove useful to have all three at our disposal. In particular, two of these regulators will explicitly break modular invariance, but are closer in spirit to those that are traditionally employed in ordinary quantum field theory. By contrast, the third regulator will be fully modular invariant. By comparing the results we will then be able to discern the novel effects that emerge through a fully modular-invariant regularization procedure and understand the reasons why such a regulator is greatly superior to the others.

All three of these regulators proceed from the same fundamental observation. Let us suppose that $F(\tau, \bar{\tau})$ diverges at least as quickly as $\tau_{2}$ as $\tau_{2} \rightarrow \infty$. Clearly, this behavior will cause the integral $I$ to diverge on the left side of Eq. (3.12). However, this behavior will also cause $g\left(\tau_{2}\right)$ to diverge as $\tau_{2} \rightarrow \infty$, which means that the right side of Eq. (3.12) will also diverge. Thus, in principle, a relation such as that in Eq. (3.12) will be rendered meaningless. However, if there were a consistent way of subtracting or regulating the appropriate divergence on each side of Eq. (3.12), we can imagine that we might then obtain an analogous relation between a finite regulated integral $\tilde{I}$ and a corresponding finite regulated physical-state trace $\tilde{g}\left(\tau_{2}\right)$. As we shall see, all three of the regulators we shall discuss have this property and lead to results which are analogous to the result in Eq. (3.12) and relate regulated integrals to regulated physical-state supertraces.

\section{Minimal regulator}

Perhaps the simplest and most minimal regulator that can be envisioned [13] is one in which we directly excise the divergence from the integral $I$ without disturbing the rest of the integral. Because the divergences on both sides of Eq. (3.12) arise as $\tau_{2} \rightarrow \infty$, we can formally excise this region of integration from $\mathcal{F}$ by defining a truncated region $\mathcal{F}_{t}$ to be the same as $\mathcal{F}$ but with the additional restriction that $\tau_{2}<t$ for some truncation cutoff $t \geq 1$. We can then define our regulated integral $\tilde{I}$ as

$$
\tilde{I} \equiv \lim _{t \rightarrow \infty}\left[\int_{\mathcal{F}_{t}} \frac{d^{2} \tau}{\tau_{2}^{2}} F-\Phi_{I}(t)\right]
$$

where the function $\Phi_{I}(t)$ describes the manner in which $\int_{\mathcal{F}_{t}} \frac{d^{2} \tau}{\tau_{2}^{2}} F$ diverges as $t \rightarrow \infty$. The explicit subtraction of $\Phi_{I}(t)$ within Eq. (3.15)—although not modular invariantthus renders $\tilde{I}$ finite.

Likewise, let us imagine that $\Phi_{g}\left(\tau_{2}\right)$ describes the manner in which $g\left(\tau_{2}\right)$ diverges as $\tau_{2} \rightarrow \infty$. We can then define

$$
\tilde{g}\left(\tau_{2}\right) \equiv g\left(\tau_{2}\right)-\Phi_{g}\left(\tau_{2}\right) .
$$

Of course, given these definitions, one might then hope that the result in Eq. (3.12) remains intact, only with the replacements $I \rightarrow \tilde{I}$ and $g\left(\tau_{2}\right) \rightarrow \tilde{g}\left(\tau_{2}\right)$. Unfortunately, things are not this simple because the $\tau_{2}<t$ truncation of $\mathcal{F}$ to $\mathcal{F}_{t}$ greatly complicates the "unfolding" procedure that underlies the intermediate algebraic step in Eq. (3.7). Starting from $\int_{\mathcal{F}_{t}} \frac{d^{2} \tau}{\tau_{2}^{2}} F$, one must therefore follow the effects of this truncation through all of the modular transformations involved in the unfolding. This procedure is performed in Ref. [13] and ultimately generates numerous extra terms beyond those appearing in Eq. (3.12). While many of these extra terms give rise to the subtractions that appear within the definitions of $\tilde{I}$ and $\tilde{g}\left(\tau_{2}\right)$ in Eqs. (3.15) and (3.16), some of these extra terms go beyond these subtractions and survive the final $t \rightarrow \infty$ limit. Thus, with $\tilde{\Phi}$ denoting these additional terms, the Rankin-Selberg result in Eq. (3.12) generalizes to take the form [13]

$$
\tilde{I}=\frac{\pi}{3} \operatorname{Res}_{s=1} \int_{0}^{\infty} d \tau_{2} \tau_{2}^{s-2} \tilde{g}\left(\tau_{2}\right)+\tilde{\Phi}
$$

where the extra terms $\tilde{\Phi}$ must also be included. We thus see that with this regulator, our original integral $I$ continues to be expressible in terms of a physical-state supertrace.

The crux of the matter, then, is to determine $\Phi_{I}(t)$, $\Phi_{g}\left(\tau_{2}\right)$, and $\tilde{\Phi}$. In general, let us suppose that $F(\tau, \bar{\tau}) \sim$ $\Phi\left(\tau_{2}\right)+\cdots$ as $\tau_{2} \rightarrow \infty$, where $\Phi\left(\tau_{2}\right)$ is a function which diverges at least as as quickly as $\tau_{2}$ as $\tau_{2} \rightarrow \infty$. Upon performing the $\tau_{1}$ integration we then immediately see that $g\left(\tau_{2}\right)$ diverges as $\tau_{2} \rightarrow \infty$ in exactly the same manner as does $F(\tau, \bar{\tau})$. We can therefore identify $\Phi_{g}\left(\tau_{2}\right)$ with $\Phi\left(\tau_{2}\right)$ itself. Likewise, it is also easy to verify that $\Phi_{I}\left(\tau_{2}\right)$ is nothing but the antiderivative of $\Phi\left(\tau_{2}\right) / \tau_{2}^{2}$, and of course 
we are free to disregard any terms within $\Phi_{I}(t)$ that vanish as $t \rightarrow \infty$ since such terms will not contribute within Eq. (3.15). The difficult part, of course, is to evaluate the extra constant term $\tilde{\Phi}$. While this term often vanishes, such a cancellation is not guaranteed. However, a general expression for this term is given in Ref. [13] for any divergence function $\Phi\left(\tau_{2}\right)$.

For our later purposes we shall only need to consider one particular case, namely that with

$$
\Phi\left(\tau_{2}\right)=c_{0}+c_{1} \tau_{2}
$$

For this divergence structure, it then follows that $\Phi_{g}=$ $c_{0}+c_{1} \tau_{2}$. Likewise, we have $\Phi_{I}(t)=-c_{0} / t+c_{1} \log t$, which we may equivalently take to be simply $\Phi_{I}(t)=$ $c_{1} \log t$ under the $t \rightarrow \infty$ limit in Eq. (3.15). Finally, for this case it turns out [13] that $\tilde{\Phi}$ is given by

$$
\begin{aligned}
\tilde{\Phi} & \equiv 2 \underset{s=1}{\operatorname{Res}}\left[\frac{c_{0} \zeta^{*}(2 s)}{s-1}-\frac{c_{1} \zeta^{*}(2 s-1)}{s-1}\right] \\
& =\frac{\pi}{3} c_{0}+\log \left(4 \pi e^{-\gamma}\right) c_{1}
\end{aligned}
$$

where $\zeta^{*}(s) \equiv \pi^{-s / 2} \Gamma(s / 2) \zeta(s)$ is the so-called "completed" Riemann $\zeta$ function and where $\gamma \approx 0.577$ is the Euler-Mascheroni constant. Thus, pulling the pieces together, we find that if $F(\tau, \bar{\tau}) \sim c_{0}+c_{1} \tau_{2}$ as $\tau \rightarrow \infty$, then our regulated integral

$$
\tilde{I} \equiv \lim _{t \rightarrow \infty}\left(\int_{\mathcal{F}_{t}} \frac{d^{2} \tau}{\tau_{2}^{2}} F-c_{1} \log t\right)
$$

can be expressed in terms of purely physical string states as

$$
\begin{aligned}
\tilde{I}= & \frac{\pi}{3} \operatorname{Res}_{s=1} \int_{0}^{\infty} d \tau_{2} \tau_{2}^{s-2}\left[g\left(\tau_{2}\right)-c_{0}-c_{1} \tau_{2}\right] \\
& +\frac{\pi}{3} c_{0}+\log \left(4 \pi e^{-\gamma}\right) c_{1} .
\end{aligned}
$$

Note that the left side of Eq. (3.20) is independent of $c_{0}$. Indeed, since the $c_{0}$ term within the asymptotic behavior of $F(\tau)$ does not actually lead to a divergence, our regulator need not depend on $c_{0}$ in any way. However, this implies that the right side of Eq. (3.21) must also be independent of $c_{0}$. We shall confirm this behavior explicitly in Sec. IV. Of course, this does not imply that we can simply set $c_{0}=0$ on the right side of Eq. (3.21); the presence of $c_{0}$ within the $\tau_{2}$ integral on the right side of Eq. (3.21) ensures that this integral diverges in precisely the correct way to yield the correct residue at $s=1$. Thus, in some sense, the appearance of $c_{0}$ within the right side of Eq. (3.21) acts precisely as a regulator should, adding a term in one place to help achieve convergence and then subtracting it somewhere else in order to yield a $c_{0}$-independent result. Note that this $c_{0}$-independence of the right side of Eq. (3.21) also allows the result in Eq. (3.21) to reduce to the RankinSelberg result in Eq. (3.12) when $c_{1}=0$ for any value of $c_{0}$. Indeed, we know that the result in Eq. (3.21) must reduce in this way because with $c_{1}=0$ we have no divergence at all, even when $c_{0} \neq 0$. The original Rankin-Selberg result in Eq. (3.12) must therefore also apply when $c_{1}=0$, even when $c_{0} \neq 0$.

\section{Nonminimal regulators}

The subtraction in Eq. (3.15) is minimal, yielding a finite result without introducing any new parameters related to the subtraction or altering any portion of the integrand other than its divergence structure. Even though a regulating parameter $t$ is introduced in Eq. (3.15), this quantity must be taken to infinity in order to encapsulate all relevant aspects of the original integral $I$.

However, it is also possible to define a similar regulator which encapsulates all parts of the original integral $I$ and yet produces a finite result for arbitrary finite values of $t \geq 1$. Since the divergences within Eq. (3.15) appear only in the $\tau_{2}>t$ region (specifically as $\tau_{2} \rightarrow \infty$ ), we can "undo" the $\tau_{2} \rightarrow \infty$ limit and alternatively define

$$
\tilde{I}(t) \equiv \int_{\mathcal{F}_{t}} \frac{d^{2} \tau}{\tau_{2}^{2}} F+\int_{\mathcal{F}_{-} \mathcal{F}_{t}} \frac{d^{2} \tau}{\tau_{2}^{2}}\left[F-\Phi\left(\tau_{2}\right)\right]
$$

where we shall continue to assume that $F(\tau, \bar{\tau}) \sim \Phi\left(\tau_{2}\right)+\cdots$ as $\tau_{2} \rightarrow \infty$. Note that $\tilde{I}(t)$ is convergent for all finite $t$, as we desire. Moreover, because the second term in Eq. (3.22) has an integrand which is convergent throughout the integration region $\mathcal{F}-\mathcal{F}_{t}$, taking the $t \rightarrow \infty$ limit eliminates the second term and $\tilde{I}(t)$ reproduces our original unregulated integral $I$ in Eq. (3.1). Thus $\tilde{I}(t)$ represents an alternative, $t$-dependent method of regulating our original integral $I$, one which is distinct from the minimal regularization $\tilde{I}$ in Eq. (3.15).

These two regularizations are deeply connected, however-a fact which will also enable us to express $\tilde{I}(t)$ in terms of supertraces, just as we did for $\tilde{I}$. Note that the only $t$-dependence within $\tilde{I}(t)$ arises from the integration of the subtraction term $\Phi\left(\tau_{2}\right)$ along the $t$-dependent lower boundary of the integration region $\mathcal{F}-\mathcal{F}_{t}$. We thus see that the subtraction term $\Phi\left(\tau_{2}\right)$ which regularizes $\tilde{I}(t)$ introduces a nontrivial dependence on $t$ such that

$$
\tilde{I}(t)=\Phi_{I}(t)+\mathcal{C}
$$

where we recall that $\Phi_{I}\left(\tau_{2}\right)$ is the antiderivative of $\Phi\left(\tau_{2}\right) / \tau_{2}^{2}$ and where $\mathcal{C}$ is an as-yet unknown $t$-independent quantity. However, it is easy to solve for $\mathcal{C}$. Given that $\mathcal{C}=\tilde{I}(t)-\Phi_{I}(t)$, we immediately see by taking the $t \rightarrow \infty$ limit of both sides and comparing with Eq. (3.15) that $\lim _{t \rightarrow \infty} \mathcal{C}=\tilde{I}$. However, $\mathcal{C}$ is independent of $t$, which means that $\mathcal{C}=\tilde{I}$ for any value of $t \geq 1$. We thus obtain a 
general relation, valid for all $t \geq 1$, between our two regulators:

$$
\tilde{I}(t)=\tilde{I}+\Phi_{I}(t) .
$$

Our previous result for $\tilde{I}$ in Eq. (3.17) then yields [13]

$$
\tilde{I}(t)=\frac{\pi}{3} \operatorname{Res}_{s=1} \int_{0}^{\infty} d \tau_{2} \tau_{2}^{s-2} \tilde{g}\left(\tau_{2}\right)+\Phi_{I}(t)+\tilde{\Phi} .
$$

Thus, just as with our minimal regulator, we find that our $t$-dependent regulator produces a finite integral $\tilde{I}(t)$ which continues to be expressible in terms of a physicalstate supertrace. Indeed, for the divergence structure $\Phi\left(\tau_{2}\right)=c_{0}+c_{1} \tau_{2}$, we find that our $t$-dependent regularized integral

$$
\tilde{I}(t) \equiv \int_{\mathcal{F}_{t}} \frac{d^{2} \tau}{\tau_{2}^{2}} F+\int_{\mathcal{F}_{-} \mathcal{F}_{t}} \frac{d^{2} \tau}{\tau_{2}^{2}}\left(F-c_{0}-c_{1} \tau_{2}\right)
$$

is given by

$$
\begin{aligned}
\tilde{I}(t)= & \frac{\pi}{3} \operatorname{Res}_{s=1} \int_{0}^{\infty} d \tau_{2} \tau_{2}^{s-2}\left[g\left(\tau_{2}\right)-c_{0}-c_{1} \tau_{2}\right] \\
& +\left(\frac{\pi}{3}-\frac{1}{t}\right) c_{0}+\log \left(4 \pi t e^{-\gamma}\right) c_{1} .
\end{aligned}
$$

Once again, $c_{0}$ plays a special role in this result because the presence of the $c_{0}$ term within $\Phi\left(\tau_{2}\right)$ does not lead to a divergence. Indeed, given that the region $\mathcal{F}-\mathcal{F}_{t}$ has volume $1 / t$ with respect to the $\frac{d^{2} \tau}{\tau_{2}^{2}}$ measure, we see that the subtraction of $c_{0}$ within Eq. (3.26) simply removes a finite quantity $c_{0} / t$ from the value of $\tilde{I}(t)$. For integrands having this divergence structure we can therefore define a modified (or improved) nonminimal regulator

$$
\begin{aligned}
\hat{I}(t) & \equiv \tilde{I}(t)+c_{0} / t \\
& =\int_{\mathcal{F}_{t}} \frac{d^{2} \tau}{\tau_{2}^{2}} F+\int_{\mathcal{F}_{-} \mathcal{F}_{t}} \frac{d^{2} \tau}{\tau_{2}^{2}}\left(F-c_{1} \tau_{2}\right),
\end{aligned}
$$

whereupon we find from Eq. (3.27) that

$$
\begin{aligned}
\hat{I}(t)= & \frac{\pi}{3} \operatorname{Res}_{s=1} \int_{0}^{\infty} d \tau_{2} \tau_{2}^{s-2}\left[g\left(\tau_{2}\right)-c_{0}-c_{1} \tau_{2}\right] \\
& +\frac{\pi}{3} c_{0}+\log \left(4 \pi t e^{-\gamma}\right) c_{1} .
\end{aligned}
$$

In other words, the $1 / t$-dependence on the right side of Eq. (3.27) was in some sense spurious, reflecting a corresponding $1 / t$-dependence that was needlessly inserted into the regulator definition in Eq. (3.26) and which has now been removed from Eqs. (3.28) and (3.29). The right side of Eq. (3.29) is then independent of $c_{0}$ in the manner discussed below Eq. (3.21) for the minimal regulator.

\section{Modular-invariant regulators}

Although our results in Eqs. (3.21), (3.27), and (3.29) were each derived in a manner that remained true to the modular-invariant unfolding procedure, neither side of these relations is modular invariant by itself. In other words, even though these relations correctly allow us to express our regulated integrals $\tilde{I}, \tilde{I}(t)$, and $\hat{I}(t)$ in terms of a corresponding regulated physical-state supertrace $\tilde{g}\left(\tau_{2}\right)$, neither $\tilde{I}, \tilde{I}(t)$, nor $\hat{I}(t)$ is itself a modular-invariant quantity. This is an important observation because these latter quantities will ultimately correspond to physical observables within the modular-invariant string context. We must therefore additionally require that these observables themselves be modular invariant.

The issue, of course, is that neither $\tilde{I}, \tilde{I}(t)$, nor $\hat{I}(t)$ incorporates a modular-invariant way of eliminating the associated divergences as $\tau_{2} \rightarrow \infty$. However, it is possible to design regulators in which such divergences are indeed eliminated in a fully modular-invariant way. In this work we shall present a particular set of modular-invariant regulators which will have several useful properties for our purposes.

In order to define these regulators, let us first recall that the partition function of a bosonic world sheet field compactified on a circle of radius $R$ is given by

$$
\begin{aligned}
Z_{\text {circ }}(a, \tau) & =\sqrt{\tau_{2}} \sum_{m, n \in \mathbb{Z}} \bar{q}^{(m a-n / a)^{2} / 4} q^{(m a+n / a)^{2} / 4} \\
& =\sqrt{\tau_{2}} \sum_{m, n \in \mathbb{Z}} e^{-\pi \tau_{2}\left(m^{2} a^{2}+n^{2} / a^{2}\right)} e^{2 \pi i m n \tau_{1}}
\end{aligned}
$$

where we have defined the dimensionless inverse radius $a \equiv \sqrt{\alpha^{\prime}} / R$. Here the sum over $m$ and $n$ represents the sum over all possible KK momentum and winding modes, respectively. Note that $Z_{\text {circ }} \rightarrow 1 / a$ as $a \rightarrow 0$, while $Z_{\text {circ }} \rightarrow a$ as $a \rightarrow \infty$. As expected, $Z_{\text {circ }}(a, \tau)$ is modular invariant for any $a$. Using $Z_{\text {circ }}(a, \tau)$, we shall then regulate any divergent integral of the form in Eq. (3.1) by defining a corresponding series of regulated integrals $\tilde{I}_{\rho}(a)$ :

$$
\tilde{I}_{\rho}(a) \equiv \int_{\mathcal{F}} \frac{d^{2} \tau}{\tau_{2}^{2}} F(\tau) \mathcal{G}_{\rho}(a, \tau)
$$

where our regulator functions $\mathcal{G}_{\rho}(a, \tau)$ are defined for any $\rho \in \mathbb{R}^{+}, \rho \neq 1$, as

$$
\mathcal{G}_{\rho}(a, \tau) \equiv A_{\rho} a^{2} \frac{\partial}{\partial a}\left[Z_{\text {circ }}(\rho a, \tau)-Z_{\text {circ }}(a, \tau)\right]
$$

where $A_{\rho} \equiv \rho /(\rho-1)$ is an overall normalization factor. Note that $\mathcal{G}_{\rho}(a, \tau)$ inherits its modular invariance from $Z_{\text {circ }}$, thereby rendering the regulated integral $\tilde{I}_{\rho}(a)$ in Eq. (3.31) fully modular invariant for any $a$ and $\rho$. We further note that $\mathcal{G}_{\rho}(a, \tau)$ satisfies the identity 


$$
\mathcal{G}_{\rho}(a, \tau)=\mathcal{G}_{1 / \rho}(\rho a, \tau) .
$$

We can therefore take $\rho>1$ without loss of generality.

These functions $\mathcal{G}_{\rho}(a, \tau)$ have two important properties which make them suitable as regulators when $a \ll 1$. First, as $a \rightarrow 0$, we find that $\mathcal{G}_{\rho}(a, \tau) \rightarrow 1$ for all $\tau$. Thus the $a \rightarrow 0$ limit restores our original unregulated theory. Second, for any $a>0$, we find that $\mathcal{G}_{\rho}(a, \tau) \rightarrow 0$ exponentially rapidly as $\tau_{2} \rightarrow \infty$. Thus the insertion of $\mathcal{G}_{\rho}(a, \tau)$ into the integrand of Eq. (3.31) successfully eliminates whatever power-law divergence might have otherwise arisen from the original integrand $F(\tau)$. Indeed, we see that this now happens in a smooth, fully modular-invariant way rather than through a sharp, discrete subtraction. Motivated by these two properties, we shall therefore focus on situations in which $a \ll 1$, as these are the situations in which our regulator preserves as much of the original theory as possible (as we expect of a good regulator) while simultaneously eliminating all power-law divergences as $\tau_{2} \rightarrow \infty$. In fact, for the special case $\rho=2$ and for the specific values $a=1 / \sqrt{k+2}$ the insertion of this regulator even has a direct physical interpretation, arising through a procedure in which the various fields in the background string geometry are turned on in such a way that the conformal field theory associated with the flat fourdimensional spacetime is replaced by that associated with a $S U(2)_{k}$ Wess-Zumino-Witten model [4-6].

That said, there is one further property of these regulator functions $\mathcal{G}_{\rho}(a, \tau)$ which will prove useful for our purposes. When $a \ll 1$, the contributions from all nonzero winding modes within $Z_{\text {circ }}$ (and ultimately within $\mathcal{G}$ ) are exponentially suppressed relative to those of the KK momentum modes. In other words, when $a \ll 1$ we can effectively restrict our summation in Eq. (3.30) to cases with $n=0$. We then find that $\mathcal{G}_{\rho}(a, \tau)$ loses its dependence on $\tau_{1}$,

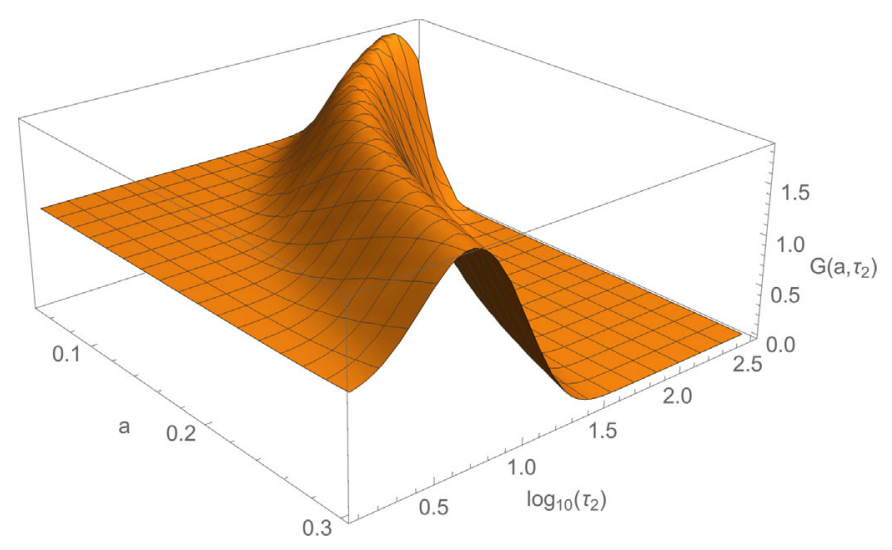

rendering $\mathcal{G}_{\rho}(a, \tau)$ a function of $\tau_{2}$ alone. In such cases we shall simply denote our regulator function as $\mathcal{G}_{\rho}\left(a, \tau_{2}\right)$.

In Fig. 1, we have plotted the regulator function $\mathcal{G}_{2}\left(a, \tau_{2}\right)$ within the $\left(a, \tau_{2}\right)$ plane for $\tau_{2} \geq 1$ (left panel) and as a function of $\tau_{2} \geq 1$ for various discrete values of $a \ll 1$ (right panel). We see, as promised, that $\mathcal{G}_{2}\left(a, \tau_{2}\right) \rightarrow 0$ for all $a>0$ as $\tau_{2} \rightarrow \infty$, while $\mathcal{G}_{2}\left(a, \tau_{2}\right) \rightarrow 1$ for all $\tau_{2} \geq 1$ as $a \rightarrow 0$. We also note that this suppression for large $\tau_{2}$ is quite pronounced, even for $a \ll 1$.

For any $a$ and $\rho$, we see from Fig. 1 that there is a corresponding value $\tau_{2}^{*}$ which can be taken as characterizing the approximate $\tau_{2}$-location of the transition between the unregulated region with $\mathcal{G}_{\rho}\left(a, \tau_{2}\right) \approx 1$ and the regulated region with $\mathcal{G}_{\rho}\left(a, \tau_{2}\right) \approx 0$. For example, we might define $\tau_{2}^{*}$ as the critical value corresponding to the top of the "ridge" in the left panel of Fig. 1 (or equivalently the maximum in the right panel of Fig. 1). Alternatively, given the shapes of the functions in the right panel of Fig. 1, we might define $\tau_{2}^{*}$ as the location at which $\mathcal{G}_{\rho}\left(a, \tau_{2}\right)$ experiences an inflection from being concave-down to concave-up. Finally, a third possibility might be to define $\tau_{2}^{*}$ as the value of $\tau_{2}$ at which $\mathcal{G}_{\rho}\left(a, \tau_{2}\right)=1 / 2$, representing the "midpoint" between $\mathcal{G}=1$ and $\mathcal{G}=0$. For the $\rho=2$ case shown in Fig. 1, we then find for $a \ll 1$ that each of these has a rather straightforward scaling behavior with $a^{-2}$ :

$$
\begin{aligned}
& \text { ridge top: } \tau_{2}^{*} \approx \frac{3}{2 \pi a^{2}} \approx \frac{0.477}{a^{2}}, \\
& \text { inflection: } \tau_{2}^{*} \approx \frac{3+\sqrt{6}}{2 \pi a^{2}} \approx \frac{0.867}{a^{2}}, \\
& \mathcal{G}=1 / 2: \tau_{2}^{*} \approx \frac{1.411}{a^{2}}
\end{aligned}
$$

Indeed, each of these results becomes increasingly accurate as $a \rightarrow 0$. Moreover, although the numerical coefficient in

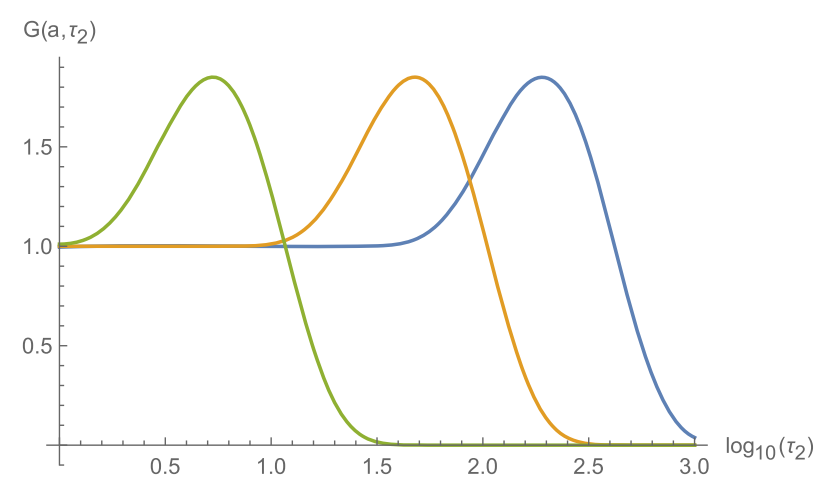

FIG. 1. Left panel: the modular-invariant regulator function $\mathcal{G}_{2}\left(a, \tau_{2}\right)$, plotted within the $\left(a, \tau_{2}\right)$ plane for $a \ll 1$ and $\tau_{2} \geq 1$. Right panel: the modular-invariant regulator $\mathcal{G}_{2}\left(a, \tau_{2}\right)$, plotted as a function of $\tau_{2}$ for $a=0.05$ (blue), $a=0.1$ (orange), and $a=0.3$ (green). In all cases we see that $\mathcal{G}_{2}\left(a, \tau_{2}\right) \rightarrow 0$ for all $a>0$ as $\tau_{2} \rightarrow \infty$, while $\mathcal{G}_{2}\left(a, \tau_{2}\right) \rightarrow 1$ for all $\tau_{2} \geq 1$ as $a \rightarrow 0$. Indeed, for $a=0.05$, we see that $\mathcal{G}_{2}\left(a, \tau_{2}\right) \approx 1$ for all $\tau_{2} \lesssim 100$. Thus for small nonzero $a$ this regulator succeeds in suppressing the divergences that might otherwise arise as $\tau_{2} \rightarrow \infty$ while nevertheless having little effect throughout the rest of the fundamental domain. 
the third case depends significantly on $\rho$, the numerical coefficients in the first two cases are actually independent of $\rho$. In all cases, however, we see that $\mathcal{G}_{\rho}\left(a, \tau_{2}\right)$ suppresses the contributions from regions of the fundamental domain with $\tau_{2} \gg \tau_{2}^{*}$ while preserving the contributions from regions with $1<\tau_{2} \ll \tau_{2}^{*}$. Indeed, this property holds regardless of our precise definition for $\tau_{2}^{*}$.

Armed with these regulator functions $\mathcal{G}_{\rho}\left(a, \tau_{2}\right)$, we now wish to express the integral in Eq. (3.31) in terms of an appropriately regulated supertrace over physical string states. However, given that $\tilde{I}_{\rho}(a)$ is fully modular invariant and convergent as $\tau_{2} \rightarrow \infty$, we can simply use the original Rankin-Selberg result in Eq. (3.12). We thus have

$$
\tilde{I}_{\rho}(a)=\frac{\pi}{3} \operatorname{Res} \int_{0=1}^{\infty} d \tau_{2} \tau_{2}^{s-2} \tilde{g}_{\rho}\left(a, \tau_{2}\right)
$$

where, in analogy with Eq. (3.5), we have

$$
\tilde{g}_{\rho}\left(a, \tau_{2}\right) \equiv \int_{-1 / 2}^{1 / 2} d \tau_{1} F(\tau) \mathcal{G}_{\rho}(a, \tau)
$$

In general, for arbitrary $a$, the regulator $\mathcal{G}_{\rho}(a, \tau)$ will have a traditional $(q, \bar{q})$ power expansion of the form $\mathcal{G} \sim \sum_{r, s} b_{r s} \bar{q}^{r} q^{s}$, just as we have $F \sim \sum_{m, n} a_{m n} \bar{q}^{m} q^{n}$ in Eq. (3.2). Given this, we find that the $\tau_{1}$ integral in Eq. (3.36) projects onto those states for which $n-m=$ $r-s$. However, $\mathcal{G}_{\rho}(a, \tau)$ generally receives contributions from states with many different values of $r-s$. As a result, $\tilde{g}_{\rho}\left(a, \tau_{2}\right)$ will generally receive contributions from not only the physical $m=n$ states within $F(\tau)$ but also some of the unphysical $m \neq n$ states. In other words, for general $a$, our regulator function $\mathcal{G}_{\rho}(a, \tau)$ becomes entangled with the physical-state trace in a way that allows unphysical states to contribute.

As we have seen, it is useful for practical purposes that $\mathcal{G}_{\rho}(a, \tau)$ loses its dependence on $\tau_{1}$ when $a \ll 1$. In other words, for $a \ll 1$ we find that the contributions from terms with $r \neq s$ within $\mathcal{G}$ are suppressed. The $\tau_{1}$ integral in Eq. (3.36) then projects onto only the $m=n$ physical states, as desired, and to a good approximation our expression for $\tilde{g}_{\rho}\left(a, \tau_{2}\right)$ in Eq. (3.36) simplifies to

$$
\tilde{g}_{\rho}\left(a, \tau_{2}\right) \approx g\left(\tau_{2}\right) \mathcal{G}_{\rho}\left(a, \tau_{2}\right)
$$

where $g\left(\tau_{2}\right)$ is our original unregulated physical-state trace in Eq. (3.5). Thus, for $a \ll 1$, the same regulator function $\mathcal{G}_{\rho}\left(a, \tau_{2}\right)$ which smoothly softens the $\tau_{2} \rightarrow \infty$ divergence in the integrand $\tilde{I}_{\rho}(a)$ also smoothly softens the $\tau_{2} \rightarrow \infty$ divergence in the physical-state trace $\tilde{g}_{\rho}\left(a, \tau_{2}\right)$-all without introducing contributions from unphysical states. However, we shall later demonstrate that the integral in Eq. (3.31) can actually be performed exactly, yielding an expression in terms of purely physical states for all values of $a$.
While these regulator functions $\mathcal{G}_{\rho}\left(a, \tau_{2}\right)$ are suitable for many applications, it turns out that we can use these functions in order to construct additional modular-invariant regulators whose symmetry properties transcend even those of $\mathcal{G}_{\rho}\left(a, \tau_{2}\right)$. To do this, we first observe from the modular invariance of $\mathcal{G}_{\rho}\left(a, \tau_{2}\right)$ that

$$
\mathcal{G}_{\rho}\left(a, 1 / \tau_{2}\right)=\mathcal{G}_{\rho}\left(a, \tau_{2}\right)
$$

for any $\rho, a$, and $\tau_{2}$. Indeed, invariance under $\tau_{2} \rightarrow 1 / \tau_{2}$ follows directly from invariance under the modular transformation $\tau \rightarrow-1 / \tau$ for $\tau_{1}=0$. Second, the identity in Eq. (3.33) tells us that the parameters $(\rho, a)$ which define our $\mathcal{G}$ functions have a certain redundancy, such that the $\mathcal{G}$ function with $(\rho, a)$ is the same as the $\mathcal{G}$ function with $(1 / \rho, \rho a)$. Indeed, only the combination $a^{\prime} \equiv \sqrt{\rho} a$ is invariant under this redundancy. Thus, while Eq. (3.38) provides a symmetry under reciprocal flips in $\tau_{2}$, Eq. (3.33) provides a symmetry under reciprocal flips in $\rho$.

Given these two symmetries, it is natural to wonder whether $\mathcal{G}_{\rho}(a, \tau)$ also exhibits a reciprocal flip symmetry in the one remaining variable $a^{\prime} \equiv \sqrt{\rho} a$. This would thus be a symmetry under $a \rightarrow 1 / \rho a$, or equivalently under $\rho a^{2} \rightarrow 1 /\left(\rho a^{2}\right)$. Indeed, we shall find in Sec. III B 4 that such an additional symmetry will be very useful and render the modular symmetry manifest in certain cases where it would otherwise have been obscure. Unfortunately, $\mathcal{G}_{\rho}(a, \tau)$ does not exhibit such a symmetry. One might nevertheless wonder whether it is possible to modify this regulator function in such a way that it might exhibit this additional symmetry as well.

It turns out that this enhanced symmetry structure is relatively easy to arrange. First, we observe that $Z_{\text {circ }}(a, \tau)$ is itself invariant under $a \rightarrow 1 / a$ for any $\tau$; indeed, this is the symmetry underlying T-duality for closed strings. Given this, it is then straightforward to verify that the functions

$$
\hat{\mathcal{G}}_{\rho}(a, \tau) \equiv \frac{1}{1+\rho a^{2}} \mathcal{G}_{\rho}(a, \tau)
$$

not only inherit all of the regulator properties and symmetries discussed above for $\mathcal{G}_{\rho}(a, \tau)$ when $a \ll 1$, but are also manifestly invariant under $a^{\prime} \rightarrow 1 / a^{\prime}$, or equivalently $a \rightarrow 1 /(\rho a)$, for any $\tau$ :

$$
\hat{\mathcal{G}}_{\rho}(a, \tau)=\hat{\mathcal{G}}_{\rho}(1 / \rho a, \tau) .
$$

We shall therefore take these $\hat{\mathcal{G}}$ functions as defining our enhanced modular-invariant regulators. We shall likewise define corresponding enhanced regularized integrals $\hat{I}_{\rho}(a)$ as in Eq. (3.31), but with $\mathcal{G}_{\rho}(a, \tau)$ replaced by $\hat{\mathcal{G}}_{\rho}(a, \tau)$. We then find that we can express $\hat{I}_{\rho}(a)$ in terms of corresponding physical-state traces $\hat{g}_{\rho}\left(a, \tau_{2}\right)$ as in 
Eqs. (3.35)-(3.37), except with $\mathcal{G}_{\rho}\left(a, \tau_{2}\right)$ replaced by $\hat{\mathcal{G}}_{\rho}\left(a, \tau_{2}\right)$ throughout.

The enhanced regulators in Eq. (3.39) can also be understood in a completely different way, through analogy with what we have already observed for our nonminimal regulators in Sec. III B 2. As discussed after Eq. (3.29), the quantity $\tilde{I}(t)$ defined through our nonminimal regulator ultimately contained a spurious $t$-dependence that could be removed without disturbing the suitability of the regulator itself. It is for this reason that we were able to transition from our original nonminimal regulator in Eq. (3.26) to our improved nonminimal regulator in Eq. (3.28) in which such spurious terms were eliminated.

It turns out that a similar situation arises for our original modular-invariant regulators $\mathcal{G}_{\rho}\left(a, \tau_{2}\right)$. Indeed, as we shall find in Sec. IV, use of these regulators would have led to results with analogously spurious terms-i.e., terms which obscure the underlying symmetries of the theory. However, just as with Eq. (3.28), it is possible to define improved modular-invariant regulators in which such spurious effects are eliminated. Indeed, these improved modular-invariant regulators are nothing but the regulators $\hat{\mathcal{G}}_{\rho}(a, \tau)$ introduced in Eq. (3.39). Additional reasons for adopting the $\hat{\mathcal{G}}_{\rho}(a, \tau)$ regulators will be discussed in Sec. VI. These improved regulators will therefore be our main interest in this paper.

\section{Aligning the nonminimal and modular-invariant regulators}

Needless to say, the most important feature of our modular-invariant regulators is precisely that they are modular invariant. Use of these regulators therefore provides a way of controlling the divergences that might appear in string amplitudes while simultaneously preserving the modular invariance that rests at the heart of all that we are doing in this paper.

This becomes especially apparent upon comparing these modular-invariant regulators with the nonminimal regulators of Sec. III B 2. Recall that the nonminimal regulators operate by isolating those terms within the partition function $F(\tau)$ which would have led to a divergence as $\tau_{2} \rightarrow \infty$, and then performing a brute-force subtraction of those terms over the entire region of the fundamental domain $\mathcal{F}$ with $\tau_{2} \geq t$. In so doing, modular invariance is broken twice: first, in artificially separating those terms within the partition function which would have led to a divergence from those which do not; and second, in then selecting a particular sharp location $\tau_{2}=t$ at which to perform the subtraction of these divergence-inducing terms, essentially multiplying these terms by $\Theta\left(t-\tau_{2}\right)$ where $\Theta$ is the Heaviside function. By contrast, our modular-invariant regulator keeps the entire partition function $F$ intact and then multiplies $F$ by a single modular-invariant regulator function $\hat{\mathcal{G}}_{\rho}(a, \tau)$. As such it does not induce a sharp
Heaviside-like subtraction at any particular location within the fundamental domain, but rather (as illustrated in the right panel of Fig. 1) induces a smooth damping which operates most strongly for $\tau_{2} \gg \tau_{2}^{*}$ and which can be removed (or pushed off toward greater and greater values of $\tau_{2}^{*}$ ) as $a \rightarrow 0$. All of these crucial differences are induced by the modular invariance of the regulator and render our modular-invariant regulators wholly different from the nonminimal regulator of Sec. III B 2 .

These two regulators do share one common feature, however: they both introduce suppressions into the integrands of our string amplitudes. Within the nonminimal regulator this takes the form of a sharp subtraction that occurs at $\tau_{2}=t$, while the modular-invariant regulator gives rise to a smoother suppression, a transition from $\hat{\mathcal{G}} \approx 1$ to $\hat{\mathcal{G}} \approx 0$ that occurs near $\tau_{2} \approx \tau_{2}^{*}$. To the extent that these two regulators share this single common feature, it is therefore possible to "align" them by choosing a particular definition for $\tau_{2}^{*}$ within the modular-invariant regulator and then identifying

$$
t=\tau_{2}^{*}
$$

In general, we have seen in Eq. (3.34) that $\tau_{2}^{*}$ takes the general form

$$
\tau_{2}^{*}=\frac{\xi}{a^{2}}=\frac{\xi \rho}{\rho a^{2}},
$$

where $\xi$ is a numerical coefficient which depends on the particular definition of $\tau_{2}^{*}$ that is chosen. Thus, for any value of $t$, we can correspondingly tune our choices for $\rho>1$ and $\rho a^{2}$ in order to enforce Eq. (3.41) and in this sense bring our regulators into alignment.

This alone is sufficient to align our regulators. However, in keeping with the spirit of symmetry enhancement that motivated our transition from $\mathcal{G}$ to $\hat{\mathcal{G}}$, we can push this one step further. We have already seen that our $\hat{\mathcal{G}}$ regulator has a symmetry under $\tau_{2} \rightarrow 1 / \tau_{2}$ (and thus under $\tau_{2}^{*} \rightarrow 1 / \tau_{2}^{*}$ ) as well as a symmetry under $a \rightarrow 1 / \rho a$ [or equivalently under $\left.\rho a^{2} \rightarrow 1 /\left(\rho a^{2}\right)\right]$. Although these are independent symmetries, the fact that $\tau_{2}^{*}$ and $\rho a^{2}$ are related through Eq. (3.42) suggests that we can align these two symmetries as well by further demanding that $\xi \rho=1$.

For either of the first two $\tau_{2}^{*}$ definitions in Eq. (3.34), this is a very easy condition to enforce: we simply take $\rho=1 / \xi$. This is possible because the "ridge-top" and "inflection" definitions lead to values of $\xi$ which are independent of $\rho$. By contrast, for the $\mathcal{G}=1 / 2$ condition (where $\mathcal{G} \approx \hat{\mathcal{G}}$ in the $\rho a^{2} \ll 1$ limit), the value of $\xi$ is itself highly $\rho$-dependent and it turns out that the constraint $\rho \xi(\rho)=1$ has no solution for $\rho$.

It is easy to understand why these different $\tau_{2}^{*}$ definitions lead to such different outcomes for $\rho$. For $a \ll 1$ and $\rho>1$, 
the contributions to $\hat{\mathcal{G}}$ from $Z_{\text {circ }}(\rho a, \tau)$ are hugely suppressed compared with those from $Z_{\text {circ }}(a, \tau)$. As a result, any defining condition for $\tau_{2}^{*}$ which depends on the actual values of $\hat{\mathcal{G}}$ will carry a sensitivity to $\rho$ only through the $\hat{\mathcal{G}}$ prefactor $A_{\rho}=\rho /(\rho-1)$. By contrast, any defining condition for $\tau_{2}^{*}$ which depends on the vanishing of derivatives of $\hat{\mathcal{G}}$ becomes insensitive to the overall scale factor $A_{\rho}$ and thus independent of $\rho$. Indeed, the "ridge-top" and "inflection" definitions depend on the vanishing of the first and second $\hat{\mathcal{G}}$-derivatives respectively. Such conditions therefore lead to a vastly simpler algebraic structure for $\tau_{2}^{*}$ as a function of $\rho$.

Thus, pulling the pieces together, we see that we can align our modular-invariant regulator with our nonminimal regulator by adopting a particular definition for $\tau_{2}^{*}$ and then choosing the values of the $(\rho, a)$ parameters within our modular-invariant regulator such that

$$
t=\frac{\xi \rho}{\rho a^{2}} .
$$

Moreover, we can further enhance the symmetries underlying this identification by restricting our attention to $(\rho, a)$ choices for which $\xi \rho=1$. However, because modular invariance essentially smoothes out the sharp transition at $\tau_{2}=t$ that otherwise existed within the nonminimal regulator, we face an inevitable uncertainty in how we define $\tau_{2}^{*}$. In the following, we shall therefore adopt

$$
t=\frac{1}{\rho a^{2}}
$$

as our alignment condition. Directly enforcing this condition enables us to sidestep the issues associated with choosing a particular value of $\xi$ or a particular definition for $\tau_{2}^{*}$. However, in enforcing this condition we should remain mindful of our regulator condition that $a \ll 1$. Likewise, whenever needed, our choices for $\rho$ should lie within a range that is sensibly close to the approximate values of $\xi^{-1}$ that characterize the transition from $\hat{\mathcal{G}} \approx 1$ to $\hat{\mathcal{G}} \approx 0$. For example, when needed for the purposes of illustration, we shall choose the fiducial value $\rho=2$. Indeed, such a value is very close to the value that would be required for the "ridge-top" definition, yielding $\xi \rho=0.954$. However, by enforcing Eq. (3.44) directly, we will be able to maintain alignment without needing to identify a particular definition for $\tau_{2}^{*}$. Moreover, as already noted, the combination $\rho a^{2}$ is invariant under the symmetry in Eq. (3.33). This combination will therefore appear naturally in many of our future calculations, thereby largely freeing us from the need to specify $\rho$ and $a$ individually.

Of course, we see from Eq. (3.44) that choosing $\rho$ within this range and taking $a \ll 1$ will be possible only if $t \gg 1$. Thus, although the choice of $t$ is completely arbitrary within the nonminimal regulator, only those nonminimal regulators with $t \gg 1$ can be aligned with our modularinvariant regulators in a meaningful way.

\section{TOWARD A FIELD-THEORETIC INTERPRETATION: THE HIGGS MASS AS A SUPERTRACE OVER PHYSICAL STRING STATES}

Equipped with the mathematical machinery from Sec. III, we now seek to express our result for the Higgs mass given in Eq. (2.55) in terms of the supertraces over only the physical string states. In so doing we will be developing an understanding of our results from a fieldtheory perspective-indeed, as a string-derived EFT valid at low energies. All of these results will be crucial for allowing us to understand how the Higgs mass "runs" within such an EFT, and ultimately allowing us to extract a corresponding "stringy" effective Higgs potential in Sec. V.

\section{A. Modular invariance, UV/IR equivalence, and the passage to an EFT}

Our first task is to understand the manner through which one may extract an EFT description of a theory with modular invariance. This is a subtle issue because such theories, as we shall see, possess a certain UV/IR equivalence. However, understanding this issue is ultimately crucial for the physical interpretations that we will be providing for our results in the rest of this section, especially as they relate to the effects of the mathematical regulators we have presented in Sec. III.

In this paper, our interest has thus far focused on performing a fully string-theoretic calculation of the Higgs mass. Given that modular invariance is a fundamental symmetry of perturbative closed strings, we have taken great care to preserve modular invariance at every step of our calculations (or to note the extent to which this symmetry has occasionally been violated, such as for two of the three possible regulators discussed in Sec. III). However, modular transformations mix the contributions of individual string states into each other in highly nontrivial ways across the entire string spectrum. Indeed, we shall see that modular invariance even leads to a fundamental equivalence between UV and IR divergences. Thus a theory such as string theory can be modular invariant only if all of its states across the entire string spectrum are carefully balanced against each other [17] and treated similarly, as a coherent whole. EFTs, by contrast, are predicated on an approach that treats UV physics and IR physics in fundamentally different ways, retaining the dynamical degrees of freedom associated with the IR physics while simultaneously "integrating out" the degrees of freedom associated with the UV physics. As a result, any attempt to develop a true EFT description of a modular-invariant theory such as string theory inherently breaks modular invariance.

It is straightforward to see that modular invariance leads to an equivalence between UV and IR divergences. 
In general, one-loop closed-string amplitudes are typically expressed in terms of modular-invariant integrands $F(\tau)$ which are then integrated over the fundamental domain $\mathcal{F}$ of the modular group. If such an amplitude diverges, this divergence will arise from the $\tau_{2} \rightarrow \infty$ region within $\mathcal{F}$. Given that the contributions from the heavy string states within the integrand are naturally suppressed as $\tau_{2} \rightarrow \infty$, it would be natural to interpret this divergence as an IR divergence involving low-energy physics.

However, such an interpretation would be inconsistent within a modular-invariant theory. In any modular-invariant theory with a modular-invariant integrand $F(\tau)$, we can always rewrite our amplitude through the identity

$$
\int_{\mathcal{F}} \frac{d^{2} \tau}{\tau_{2}^{2}} F(\tau)=\int_{\mathcal{F}} \frac{d^{2} \tau}{\tau_{2}^{2}} F(\gamma \cdot \tau)=\int_{\gamma \cdot \mathcal{F}} \frac{d^{2} \tau}{\tau_{2}^{2}} F(\tau)
$$

which holds for any modular transformation $\gamma$. From Eq. (4.1) we see that choosing $\mathcal{F}$ as our region of integration is mathematically equivalent to choosing any of its images $\gamma \cdot \mathcal{F}$ under any modular transformation $\gamma$. One of these equivalent choices is $\mathcal{F}^{\prime} \equiv \gamma_{S} \cdot \mathcal{F}$ where $\gamma_{S}$ is the $\tau \rightarrow-1 / \tau$ modular transformation. This region is explicitly given as

$$
\begin{aligned}
\mathcal{F}^{\prime} \equiv\left\{\tau: \tau_{2}>0,|\tau| \leq 1,\left(\tau_{1}+1\right)^{2}+\tau_{2}^{2} \geq 1,\right. \\
\\
\left.\left(\tau_{1}-1\right)^{2}+\tau_{2}^{2} \geq 1\right\},
\end{aligned}
$$

and as such includes the $\tau_{2} \rightarrow 0$ region but no longer includes the $\tau_{2} \rightarrow \infty$ region. Indeed, via the identity in Eq. (4.1) we see that the divergence of our amplitude now appears as $\tau_{2} \rightarrow 0$. However, there is no suppression of the contributions from the heavy string states within the integrand as $\tau_{2} \rightarrow 0$. Instead, any divergence as $\tau_{2} \rightarrow 0$ arises through the accumulating contributions of the heavy string states and would therefore naturally be interpreted as a UV divergence. Thus, by trading $\mathcal{F}$ for $\mathcal{F}^{\prime}$ through Eq. (4.1), we see that we can always mathematically recast what would naively appear to be an IR divergence as $\tau_{2} \rightarrow \infty$ into what would naively appear to be a UV divergence as $\tau_{2} \rightarrow 0$-all without disturbing the integrand of our amplitude in any way. A similar conclusion holds for the many other $\mathcal{F}^{\prime \prime}$ domains that could equivalently have been chosen for other choices of the modular transformation $\gamma$.

This is a fundamental observation. When we calculate an amplitude in string theory, we are equipped with an integrand which reflects the spectrum of string states but we must choose an appropriate fundamental domain of the modular group. This choice is not something dictated within the theory itself, but instead amounts to a convention which is adopted for the sake of performing a calculation. It is possible, of course, that the amplitude in question diverges. As we have seen, if we choose the fundamental domain $\mathcal{F}$ as defined in Eq. (2.24) then this divergence will manifest itself as an IR divergence. However, if we choose $\mathcal{F}^{\prime}$ as our fundamental domain, this same divergence of the amplitude will manifest itself as a UV divergence. Both interpretations are equally valid because the divergence of a one-loop modular-invariant string amplitude is neither intrinsically UV nor intrinsically IR. Indeed, such a divergence is a property of the amplitude itself and is not intrinsically tied to any particular value of $\tau$. Such a divergence is then merely represented as a UV or IR divergence depending on our choice of a region of integration.

This observation can also be understood through a comparison with our expectations from quantum field theory. As we have seen in Eq. (3.7), there is a tight relation between the fundamental domain $\mathcal{F}$ and the strip $\mathcal{S}$ defined in Eq. (3.3): essentially $\mathcal{F}$ is a "folded" version of $\mathcal{S}$. Likewise, the modular-invariant integrand $F(\tau)$ that is integrated over $\mathcal{F}$ is nothing but the sum of the images of the noninvariant integrand which would be integrated over $\mathcal{S}$. Thus, through the unfolding procedure in Eq. (3.7), we have two equivalent representations for the same physics. These are often called the $\mathcal{F}$ - and $\mathcal{S}$-representations.

It is through the $\mathcal{S}$-representation that we can most directly make contact with the results that would come from a quantum field theory based on point particles. Within the $\mathcal{S}$-representation, we can identify $\tau_{2}$ as the Schwinger proper-time parameter, with $\tau_{2} \rightarrow \infty$ corresponding to the field-theoretic IR limit and with $\tau_{2} \rightarrow 0$ corresponding to the field-theoretic UV limit. Indeed, within field theory these limits are physically distinct, just as they are geometrically distinct within the strip. However, upon folding the strip $\mathcal{S}$ into the fundamental domain $\mathcal{F}$, we see that both the UV and IR field-theoretic regions within $\mathcal{S}$ are together mapped onto the $\tau_{2} \rightarrow \infty$ region within $\mathcal{F}$. Indeed, the distinct UV and IR regions of the strip $\mathcal{S}$ are now "folded" so as to lie directly on top of each other within $\mathcal{F}$. Thus, within the $\mathcal{F}$-representation, the $\tau_{2} \rightarrow \infty$ limit in some sense represents both the UV and IR field-theory limits simultaneously-limits which would have been viewed as distinct within field theory but which are now related to each other in string theory through modular invariance. An identical argument also holds for the $\tau_{2} \rightarrow 0$ region within $\mathcal{F}^{\prime}$.

We can therefore summarize the situation as follows. For a modular-invariant string-theoretic amplitude there is only one kind of divergence. It can be represented as either a UV divergence or an IR divergence depending on our choice of fundamental domain (region of integration). However, in either case, this single string-theoretic divergence can be mapped back to what can be considered a modularinvariant combination of UV and IR field-theoretic divergences in field theory (i.e., on the strip $\mathcal{S}$ ). Indeed, we may schematically write

$$
\underbrace{\mathrm{IR}_{\mathcal{F}}=\mathrm{UV}_{\mathcal{F}^{\prime}}}_{\text {string theory }} \Leftrightarrow \underbrace{\mathrm{IR}_{\mathcal{S}} \oplus \mathrm{UV}_{\mathcal{S}}}_{\text {field theory }}
$$


where " $\oplus$ " signifies a modular-invariant combination. We shall obtain an explicit example of such a combination below. It is ultimately in this way, through Eq. (4.3), that our modular-invariant string theory loses its ability to distinguish between UV and IR physics. We will discuss these issues further in Sec. VI.

Our discussion in this paper has thus far been formulated with $\mathcal{F}$ chosen as our fundamental domain. In this way we have been implicitly casting our string-theoretic divergences as infrared. In the following, we shall therefore continue along this line and attach corresponding physical interpretations to our mathematical results as far as possible. However, we shall also occasionally indicate how our results might alternatively appear within the $\mathcal{F}^{\prime}$-representation, or within the unfolded $\mathcal{S}$-representation of ordinary quantum field theory. This will ultimately be important for extracting an EFT for the Higgs mass, for understanding how our Higgs mass "runs" within such an EFT, and for eventually interpreting our results in terms of a stringy effective potential.

One further comment regarding the nature of these divergences is in order. The above discussion has focused on the manner in which modular invariance mixes UV and IR divergences when passing from field theory to string theory. However, it is also important to remember that modular invariance likewise affects the strengths of these divergences. To understand this, we recall from Eq. (3.6) that the strip $\mathcal{S}$, which serves as the field-theoretic region of integration, is nothing but the sum of the images of $\mathcal{F}$, a string-theoretic region of integration, under each of the modular transformations $\gamma$ in the coset $\Gamma_{\infty} \backslash \Gamma$. However, there is an infinite number of such modular transformations within this coset. This means, in essence, that our stringtheoretic divergences (if any) are added together an infinite number of times when $\mathcal{F}$ is unfolded into $\mathcal{S}$, implying that the resulting field-theoretic divergences are far more severe than those of the string. Phrased somewhat differently, we see that modular invariance softens a given field-theoretic divergence by allowing us to reinterpret part of this divergence as resulting from an infinity of identical copies of a weaker (modular-invariant) string divergence, whereupon we are authorized to select only one such copy.

This observation is completely analogous to what happens within field theory in the presence of a gauge symmetry. If we were to disregard the gauge symmetry when calculating a field-theoretic amplitude, we would integrate over an infinite number of gauge slices when performing our path integrals. This would result in divergences which are spuriously severe. However, modular invariance is similar to gauge symmetry in the sense that both represent redundancies of description. (In the case of modular invariance, the redundancy arises from the fact that all values of $\gamma \cdot \tau$ for $\gamma \in \Gamma$ correspond to the same world sheet torus.) In a modular-invariant theory, we therefore divide out by the (infinite) volume of the redundancy coset
$\Gamma_{\infty} \backslash \Gamma$ and consider only one modular-invariant "slice." Indeed, this is precisely what is happening when we pass from $\mathcal{S}$ to $\mathcal{F}$ (or any of its images) as the appropriate region of integration in a modular-invariant theory, where the particular choice of image is nothing but the particular choice of slice. This passage from $\mathcal{S}$ to a particular modularinvariant slice then softens our field-theoretic divergences and in some cases even eliminates them entirely.

We have already seen one example of this phenomenon: the one-loop vacuum energy (cosmological constant) $\Lambda$ is badly divergent in quantum field theory, yet finite in any tachyon-free closed-string theory. Indeed, it is modular invariance which is alone responsible for this phenomenon. As we shall see, a similar softening of divergences also occurs for the Higgs mass.

We conclude this discussion with one additional comment. It is a common assertion that string theory lacks UV divergences. The rationale usually provided for this is that string theory intrinsically has a minimum length scale, namely $M_{s}^{-1}$, and that this provides a "cutoff" that eliminates all physics from arbitrarily short length scales and thereby eliminates the associated UV divergences. However, this argument fails to acknowledge that IR divergences may still remain, and of course in a modularinvariant theory the UV and IR divergences are mixed. Indeed, as we have explained, there is no modular-invariant way of disentangling these two kinds of divergences. Thus string theory is not free of divergences. These divergences are simply softer than they would have been in field theory.

\section{B. The divergence structure of the Higgs mass}

With the above comments in mind, we now consider the divergence structure of the Higgs mass. We begin by recalling from Eq. (2.55) that the Higgs mass $m_{\phi}$ within any four-dimensional heterotic string is given by

$m_{\phi}^{2}=-\frac{\mathcal{M}^{2}}{2}\left(\left\langle\mathcal{X}_{1 a}\right\rangle+\left\langle\mathcal{X}_{1 b}\right\rangle+\left\langle\mathcal{X}_{2}\right\rangle\right)+\frac{\xi}{4 \pi^{2}} \frac{\Lambda}{\mathcal{M}^{2}}$

where

$$
\begin{aligned}
\mathcal{X}_{1 a} & \equiv \frac{\tau_{2}}{\pi}\left(\tilde{\mathbf{Q}}_{j}^{t} \mathbf{Q}_{h}+\mathbf{Q}_{j}^{t} \tilde{\mathbf{Q}}_{h}\right), \\
\mathcal{X}_{1 b} & \equiv-\frac{\tau_{2}}{\pi}\left(\mathbf{Q}_{h}^{2}+\tilde{\mathbf{Q}}_{h}^{2}\right), \\
\mathcal{X}_{2} & \equiv \tau_{2}^{2}\left(\mathbf{Q}_{R}^{t} \mathbf{Q}_{h}-\mathbf{Q}_{L}^{t} \tilde{\mathbf{Q}}_{h}\right)^{2} \\
& =4 \tau_{2}^{2}\left(\mathbf{Q}_{R}^{t} \mathbf{Q}_{h}\right)^{2}=4 \tau_{2}^{2}\left(\mathbf{Q}_{L}^{t} \tilde{\mathbf{Q}}_{h}\right)^{2}
\end{aligned}
$$

and where $\Lambda$ is the one-loop cosmological constant. Note that we have explicitly separated those terms $\mathcal{X}_{1 a}$ and $\mathcal{X}_{1 b}$ which are quadratic in charge insertions from those terms $\mathcal{X}_{2}$ which are quartic, as these will shortly play very different roles. Moreover, within the quadratic terms, we have further distinguished those insertions $\mathcal{X}_{1 a}$ within 
which each term consists of a paired contribution of a leftmoving charge with a right-moving charge from those insertions $\mathcal{X}_{1 b}$ in which each term consists of two charges which are both either left- or right-moving. Indeed, we recall from Sec. II that only $\left\langle\mathcal{X}_{1 a}\right\rangle$ and the sum $\left\langle\mathcal{X}_{1 b}\right\rangle+\left\langle\mathcal{X}_{2}\right\rangle$ are modular invariant; in particular, $\left\langle\mathcal{X}_{1 b}\right\rangle$ and $\left\langle\mathcal{X}_{2}\right\rangle$ are the modular completions of each other and thus neither is modular invariant by itself. That said, it will prove convenient in this section to simply define

$$
\begin{aligned}
\mathcal{X}_{1} & \equiv \mathcal{X}_{1 a}+\mathcal{X}_{1 b} \\
& =\frac{\tau_{2}}{\pi}\left(\tilde{\mathbf{Q}}_{j}^{t} \mathbf{Q}_{h}+\mathbf{Q}_{j}^{t} \tilde{\mathbf{Q}}_{h}-\mathbf{Q}_{h}^{2}-\tilde{\mathbf{Q}}_{h}^{2}\right),
\end{aligned}
$$

so long as we remember that only the full combination $\left\langle\mathcal{X}_{1}\right\rangle+\left\langle\mathcal{X}_{2}\right\rangle$ is modular invariant.

As discussed in Sec. II, these results are completely general and apply to any scalar $\phi$ whose VEV determines the vacuum structure of the theory. Indeed, the various charge insertions $\mathbf{Q}_{h}, \tilde{\mathbf{Q}}_{h}, \mathbf{Q}_{j}$, and $\tilde{\mathbf{Q}}_{j}$ in Eq. (4.5) are defined in Eqs. (2.30) and (2.31) in terms of the $\mathcal{T}$-matrices which encapsulate the relevant information concerning specific scalar under study.

Unlike the other terms in Eq. (4.4), the final term $\Lambda$ emerges as the result of a universal shift in the background moduli. As such, this quantity is wholly independent of the specific $\mathcal{T}$-matrices, and merely provides a uniform shift to the masses of all scalars in the theory regardless of the specific roles these scalars might play in breaking gauge symmetries or otherwise affecting the vacuum state of the theory. In other words, $\Lambda$ provides what is essentially a mere "background" contribution to our scalar masses. Moreover, as the one-loop cosmological constant of the theory, $\Lambda$ is an independent physical observable unto itself. For this reason, we shall defer our discussion of $\Lambda$ to Sec. IV E 2 and focus instead on the effects coming from the $\mathcal{X}_{i}$ insertions in Eq. (4.4).

In order to make use of the machinery in Sec. III, we must first understand the divergence structure that can arise from each of these $\mathcal{X}_{i}$ insertions as $\tau_{2} \rightarrow \infty$. For any string model in four spacetime dimensions, the original partition function prior to any $\mathcal{X}_{i}$ insertions has the form indicated in Eq. (2.1), with an overall factor of $\tau_{2}^{-1}$. Thus, the insertion of $\mathcal{X}_{1}$ leads to integrands without a leading factor of $\tau_{2}$, while the insertion of $\mathcal{X}_{2}$ leads to integrands with a leading factor of $\tau_{2}^{+1}$.

Determining the possible divergences as $\tau_{2} \rightarrow \infty$ requires that we also understand the spectrum of low-lying states that contribute to these integrands. We shall, of course, assume that our string model is free of of physical (on-shell) tachyons. Thus, expanding the partition function $\mathcal{Z}$ of our string model as in Eq. (3.2) with $k=-1$, we necessarily have $a_{n n}=0$ for all $n<0$ in Eq. (3.2).

There is, however, an off-shell tachyonic state which must always appear within the spectrum of any self-consistent heterotic string model: this is the so-called proto-graviton [9] with $(m, n)=(0,-1)$, and no possible Gliozzi-ScherkOlive projection can eliminate this state from the spectrum. Although this state is necessarily a singlet under all of the gauge symmetries of the model, it transforms as a vector under the spacetime Lorentz group. Consequently the degrees of freedom that compose this state have nonvanishing charge vectors of the form

$$
\mathbf{Q}_{\text {proto-graviton }}=\left(\mathbf{0}_{22} \mid \pm 1, \mathbf{0}_{9}\right)
$$

where we have written this charge vector in the same basis as used in Eq. (2.21), with the nonzero charge component in Eq. (4.7) lying along the spacetime-statistics direction discussed in Sec. II C.

Because of this nonzero charge component, the protograviton state has the possibility of contributing to one-loop string amplitudes even when certain charge insertions occur. However, we have seen in Eq. (2.21) that the $\mathcal{T}$-matrices appropriate for shifts in the Higgs VEV do not disturb the spin-statistics of the states in the spectrum, and thus necessarily have zeros along the corresponding columns and rows. Indeed, these zeros are a general feature which would apply to all such $\mathcal{T}$-matrices regardless of the specific Higgs scalar under study or its particular gauge embedding. As a result, the would-be contributions from the proto-graviton state do not survive either of the $\mathcal{X}_{i}$ insertions in Eq. (4.5). Indeed, similar arguments also apply to potential contributions from the proto-gravitino states (such as would appear in the spectra of string models exhibiting spacetime supersymmetry).

In general, a heterotic-string model can also contain other off-shell tachyonic $(m, n)$ states with $m \neq n$ but $m+n<0$. Unlike the proto-graviton state with $(m, n)=(0,-1)$, such states would generally have $(m, n)=(k+1, k)$ where $-1<k<-1 / 2$. However, even if a given string model were to contain such states, these states-like the protograviton state-would also likely not have nonzero charges in the appropriate Higgs directions. Indeed, this closely mirrors the situation that emerges for the analogous calculation of string threshold corrections in a variety of semirealistic string models, as discussed in Ref. [26], where it was explicitly demonstrated that none of the potential offshell tachyonic states that appeared in such models carried the sorts of nontrivial gauge charges that were relevant for the corresponding gauge threshold calculations. We shall therefore make the same assumption here.

The net result, then, is that the lightest states that can contribute in the presence of the $\mathcal{X}_{i}$ insertions are the massless on-shell string states. These states contribute to the $(m, n)=(0,0)$ term in the integrand, and thus their contributions cause the integrand to scale as $\tau_{2}^{k}$ in the $\tau_{2} \rightarrow \infty$ limit, where $k=0$ for the $\mathcal{X}_{1}$ insertion and $k=1$ for the $\mathcal{X}_{2}$ insertion. Moreover, all heavier states make contributions which are exponentially suppressed as 
$\tau_{2} \rightarrow \infty$, regardless of the $\tau_{2}^{k}$ prefactor, and thus do not lead to divergences in this limit. We thus see that the $\mathcal{X}_{1}$ and $\mathcal{X}_{2}$ insertions together produce an integrand exhibiting the $\tau_{2} \rightarrow \infty$ divergence structure given in Eq. (3.18), where we now identify

$$
\begin{aligned}
& c_{0}=-\frac{1}{2} \mathcal{M}^{2} \underset{M=0}{\operatorname{Str}} \mathbb{X}_{1}, \\
& c_{1}=-\frac{1}{2} \mathcal{M}^{2} \underset{M=0}{\operatorname{Str} \mathbb{X}_{2} .}
\end{aligned}
$$

Here the $\mathbb{X}_{i}$ are the same as the charge insertions $\mathcal{X}_{i}$ but without their leading $\tau_{2}$ prefactors:

$$
\mathbb{X}_{1} \equiv \tau_{2}^{-1} \mathcal{X}_{1}, \quad \mathbb{X}_{2} \equiv \tau_{2}^{-2} \mathcal{X}_{2}
$$

Likewise, in writing the expressions in Eq. (4.8) we are introducing the supertrace notation

$$
\operatorname{Str} A \equiv \sum_{\text {physical } i}(-1)^{F_{i}} A_{i}
$$

where the sum is over the physical states $i$ in the string spectrum, where $F_{i}$ is the spacetime fermion number of state $i$, and where $A_{i}$ is the value of $A$ for that state. Note that the off-shell string states with $m \neq n$ are not propagating string degrees of freedom and thus our definition for the supertrace "Str" in Eq. (4.10) does not include them. The supertrace "Str" therefore includes the contributions from only those string states which have direct field-theory analogs. In Eq. (4.8) the " $M=0$ " subscripts on "Str" indicate that these supertraces are further restricted to include only those states which are massless. We thus see that the divergence in the Higgs mass arises, not unexpectedly, from the contributions of massless string states (specifically those massless states which are charged under the different $\mathbb{X}_{i}$ ). This is exactly as we expect for an infrared divergence. However, as discussed in Sec. IVA, the interpretation of this divergence as being infrared in nature and arising from massless states depends crucially on our choice to work in the $\mathcal{F}$-representation.

Given the divergence structure in Eq. (3.18) with coefficients given in Eq. (4.8), we see that our expression for the Higgs mass in Eq. (4.4) is generically logarithmically divergent as $\tau_{2} \rightarrow \infty$ (and finite only if $c_{1} \sim \underset{M=0}{\operatorname{Str}} \mathbb{X}_{2}$ happens to vanish within a particular string model). We thus see that

- Just as the one-loop vacuum energy in any tachyonfree closed-string theory is finite as a result of modular invariance, the corresponding Higgs mass is at most logarithmically divergent.

Modular invariance has thus induced a significant softening of the Higgs divergence, reducing what would have been a quadratic UV Higgs divergence in field theory into a logarithmic Higgs divergence in string theory. However, even though the Higgs divergence has been softened within Eq. (4.4), we must still regulate the logarithmic divergence that remains. In the following we shall do this using the regulators discussed in Sec. III and interpret the resulting expressions in terms of an EFT. Because each of these regulators has different strengths and weaknesses, we shall apply each of these regulators in turn. Comparing the corresponding results will ultimately enable us to understand the full power of the modular-invariant regulator.

\section{Results using the minimal regulator}

Using the minimal regulator discussed in Sec. III B 1, we can regulate the Higgs mass in Eq. (4.4) to take the form

$$
\begin{aligned}
\tilde{m}_{\phi}^{2}= & -\frac{1}{2} \mathcal{M}^{2} \lim _{t \rightarrow \infty}\left[\left\langle\mathcal{X}_{1}\right\rangle_{t}+\left\langle\mathcal{X}_{2}\right\rangle_{t}\right. \\
& \left.-\left(\operatorname{Str}_{M=0} \mathbb{X}_{2}\right) \log t\right]+\frac{\xi}{4 \pi^{2}} \frac{\Lambda}{\mathcal{M}^{2}}
\end{aligned}
$$

where, in analogy with Eq. (2.52), we have now defined

$$
\langle A\rangle_{t} \equiv \int_{\mathcal{F}_{t}} \frac{d^{2} \tau}{\tau_{2}^{2}} \frac{\tau_{2}^{-1}}{\bar{\eta}^{12} \eta^{24}} \sum_{\mathbf{Q}_{L}, \mathbf{Q}_{R}}(-1)^{F} A \bar{q}^{\mathbf{Q}_{R}^{2} / 2} q^{\mathbf{Q}_{L}^{2} / 2}
$$

where $\mathcal{F}_{t}$ is the truncated domain of integration discussed above Eq. (3.15). Note that unlike $m_{\phi}^{2}$ in Eq. (4.4), our expression for the regulated $\tilde{m}_{\phi}^{2}$ is manifestly finite, as the logarithmic divergence arising due to the massless string states charged under $\mathcal{X}_{2}$ has been explicitly excised. Indeed, this explicit subtraction of the contributions from massless states is similar to what occurs in the calculation of stringtheoretic gauge threshold corrections in Ref. [1] and in the calculation of string-theoretic kinetic mixing in Ref. [27].

While the expectation values $\left\langle\mathcal{X}_{i}\right\rangle$ in Eq. (4.11) receive contributions from both physical and unphysical string states, our results from Sec. III B 1 allow us to express $\tilde{m}_{\phi}^{2}$ purely in terms of physical string states. Indeed, disregarding the contributions from the finite $\Lambda$-term in Eq. (4.4), we find that our unregulated physical-state trace $g\left(\tau_{2}\right)$ is given by

$$
g\left(\tau_{2}\right)=-\frac{1}{2} \mathcal{M}^{2}\left[\operatorname{Str}\left(\mathbb{X}_{1}+\tau_{2} \mathbb{X}_{2}\right) e^{-\pi \alpha^{\prime} M^{2} \tau_{2}}\right],
$$

consistent with the divergence structure $\Phi_{g}\left(\tau_{2}\right)=c_{0}+c_{1} \tau_{2}$ discussed above. Our regulated physical-state trace $\tilde{g}\left(\tau_{2}\right)$ in Eq. (3.16) is then given by

$$
\begin{aligned}
\tilde{g}\left(\tau_{2}\right) \equiv & -\frac{1}{2} \mathcal{M}^{2}\left\{\operatorname{Str}\left[\left(\mathbb{X}_{1}+\tau_{2} \mathbb{X}_{2}\right) e^{-\pi \alpha^{\prime} M^{2} \tau_{2}}\right]\right. \\
& \left.-\underset{M=0}{\operatorname{Str}} \mathbb{X}_{1}-\left(\underset{M=0}{\operatorname{Str}} \mathbb{X}_{2}\right) \tau_{2}\right\} \\
= & -\frac{1}{2} \mathcal{M}^{2} \underset{M>0}{\operatorname{Str}}\left[\left(\mathbb{X}_{1}+\tau_{2} \mathbb{X}_{2}\right) e^{-\pi \alpha^{\prime} M^{2} \tau_{2}}\right],
\end{aligned}
$$


and thus we see that only the massive string states contribute to $\tilde{g}\left(\tau_{2}\right)$. However, integrating our result for $\tilde{g}\left(\tau_{2}\right)$ in Eq. (4.14) over $\tau_{2}$ and taking the residue at $s=1$ as in Eq. (3.21), we find

$$
\begin{aligned}
\underset{s=1}{\operatorname{Res}} \int_{0}^{\infty} d \tau_{2} \tau_{2}^{s-2} \tilde{g}\left(\tau_{2}\right) \\
=-\frac{1}{2} \mathcal{M}^{2} \operatorname{Res}_{s=1}\left\{\Gamma(s-1) \underset{M>0}{\operatorname{Str}}\left[\mathbb{X}_{1}\left(\pi \alpha^{\prime} M^{2}\right)^{1-s}\right]\right. \\
\left.\quad+\Gamma(s) \underset{M>0}{\operatorname{Str}}\left[\mathbb{X}_{2}\left(\pi \alpha^{\prime} M^{2}\right)^{-s}\right]\right\} \\
=-\frac{1}{2} \mathcal{M}^{2} \operatorname{Str}_{M>0} \mathbb{X}_{1} .
\end{aligned}
$$

Thus taking the residue has the effect of further projecting out from the $\tilde{g}\left(\tau_{2}\right)$ term the contributions that emerge from the $\mathbb{X}_{2}$ insertion. Following Eq. (3.21) we then obtain our final result for the regulated Higgs mass:

$$
\begin{aligned}
\tilde{m}_{\phi}^{2} & =-\frac{1}{2} \mathcal{M}^{2}\left[\frac{\pi}{3} \underset{M>0}{\operatorname{Str}} \mathbb{X}_{1}+\frac{\pi}{3} \operatorname{Str}_{M=0} \mathbb{X}_{1}+\left(\underset{M=0}{\operatorname{Str}} \mathbb{X}_{2}\right) \log 4 \pi e^{-\gamma}\right] \\
& =-\frac{1}{2} \mathcal{M}^{2}\left[\frac{\pi}{3} \operatorname{Str} \mathbb{X}_{1}+\left(\underset{M=0}{\operatorname{Str}} \mathbb{X}_{2}\right) \log 4 \pi e^{-\gamma}\right]
\end{aligned}
$$

where we have not displayed the additional universal $\Lambda$-term that appears in Eq. (4.11). Note, in particular, that the contributions from the massless states within the $\mathbb{X}_{1}$ insertion, after having been subtracted in Eq. (4.14), have been restored in Eq. (4.16). This is precisely in accord with our expectations regarding the role of the $c_{0}$ parameter, as discussed below Eq. (3.21).

One important comment is in order. In our derivation in Eq. (4.15), we implicitly assumed that the residue of the supertrace sum is the same as the supertrace sum of the individual residues. This allowed us to exchange the order of the supertrace-summation and residue-extraction procedures. This kind of calculation will occur repeatedly throughout this paper, and we shall do this in each instance. This exchange of ordering is justified because we are working within a regulated theory in which there are no additional divergences that might arise from the supertrace sum beyond those which were already encapsulated within our original assertion that the Higgs mass has at most a logarithmic divergence when $\underset{M=0}{\operatorname{Str}} \mathbb{X}_{2} \neq 0$, or equivalently that $g\left(\tau_{2}\right)$ diverges no more rapidly than $c_{0}+c_{1} \tau_{2}$ as $\tau_{2} \rightarrow \infty$. This will be discussed further in Sec. VI.

We see, then, that use of the minimal regulator discussed in Sec. III B 1 leads to a final parameter-independent expression for the Higgs mass purely in terms of the contributions of physical string states. Moreover, this expression eliminates the explicit contributions from the massive states within the quartic insertion $\mathbb{X}_{2}$. This initially surprising observation makes sense if we think of the logarithmic divergence of $m_{\phi}^{2}$ as an ultraviolet one, given that the contributions of the massive states to the quartic terms are the contributions which are expected to grow the most rapidly as we proceed upward through string spectrum.

Despite the form of Eq. (4.16), we note that $\tilde{m}_{\phi}^{2}$ is not actually insensitive to the massive spectrum resulting from the $\mathcal{X}_{2}$ insertion, just as $\tilde{m}_{\phi}^{2}$ is not insensitive to all of the unphysical string states whose contributions also originally appeared within Eq. (4.11). Indeed all of these statesalong with the full spectrum of physical states from the $\mathcal{X}_{1}$ insertion - are part of what makes our original expression for $m_{\phi}^{2}$ invariant under the fundamental modular symmetry which lies at the core of the procedure we have followed in casting $\tilde{m}_{\phi}^{2}$ as a supertrace over only physical string states. Indeed, as we have already seen in Sec. II E, the insertion $\mathcal{X}_{1}$ is intrinsically part of the modular completion of $\mathcal{X}_{2}$, and vice versa. We should therefore simply interpret the result in Eq. (4.16) as telling us that modular invariance is a sufficiently powerful symmetry that the spectra resulting from the $\mathcal{X}_{1}$ and $\mathcal{X}_{2}$ insertions are no longer independently adjustable, but rather are so locked together that it is no longer necessary to explicitly sum over all of them independently when expressing our regulated Higgs mass $\tilde{m}_{\phi}^{2}$ in this manner.

That said, it is significant that our regulated result for the Higgs mass in Eq. (4.16) treats $\mathbb{X}_{1}$ and $\mathbb{X}_{2}$ in fundamentally different ways. As noted below Eq. (4.5), only the strict combination $\mathcal{X}_{1}+\mathcal{X}_{2}$ preserves modular invariance. Indeed, $\mathcal{X}_{1}$ and $\mathcal{X}_{2}$ appeared symmetrically in our original unregulated expression in Eq. (4.4). We therefore see that although the string spectrum itself is strictly modular invariant, as discussed above, our regulated result for the Higgs mass in Eq. (4.16) is not. Of course, this outcome is completely expected because our regulator in this case is built upon a method for subtracting divergences which is not modular invariant.

\section{Results using the nonminimal regulator}

Let us now investigate how these results change if we instead regulate our Higgs mass using the modified nonminimal regulator of Eqs. (3.28) and (3.29). As discussed in Sec. III, this regulator-like the minimal regulator-is also not modular invariant. However, it will lead to a richer structure than that obtained from the minimal regulator-a structure which will enable us to make a comparison with field-theoretic expectations.

Using the modified nonminimal regulator, we follow Eq. (3.28) in defining the regulated form of our logarithmically divergent Higgs mass $m_{\phi}^{2}$ as

$$
\begin{aligned}
\hat{m}_{\phi}^{2}(t) \equiv & -\frac{1}{2} \mathcal{M}^{2}\left[\left\langle\mathcal{X}_{1}\right\rangle+\left\langle\mathcal{X}_{2}\right\rangle-\int_{\mathcal{F}-\mathcal{F}_{t}} \frac{d^{2} \tau}{\tau_{2}^{2}} \tau_{2}\left(\underset{M=0}{\left.\left.\operatorname{Str} \mathbb{X}_{2}\right)\right]}\right.\right. \\
& +\frac{\xi}{4 \pi^{2}} \frac{\Lambda}{\mathcal{M}^{2}} .
\end{aligned}
$$


Unlike our expression for $\tilde{m}_{\phi}^{2}$ in Eq. (4.11) which employed the minimal regulator, we see that this new regulated Higgs mass $\hat{m}_{\phi}^{2}(t)$ is a function of an arbitrary dimensionless parameter $1 \leq t<\infty$. Given the discussion in Sec. III B 2, along with our result for the minimal regulator in Eq. (4.16), it then follows that $\hat{m}_{\phi}^{2}(t)$ can be expressed purely in terms of physical string states as

$\hat{m}_{\phi}^{2}(t)=-\frac{1}{2} \mathcal{M}^{2}\left[\frac{\pi}{3} \operatorname{Str} \mathbb{X}_{1}+\left(\underset{M=0}{\operatorname{Str}} \mathbb{X}_{2}\right) \log 4 \pi t e^{-\gamma}\right]$

where we have again refrained from displaying the additional universal $\Lambda$-term. Indeed, the extra crucial factor within Eq. (4.18) relative to the result in Eq. (4.16) is a new logarithmic dependence on the regulator parameter $t$.

The expression for $\hat{m}_{\phi}^{2}(t)$ in Eq. (4.18) is the exact stringtheoretic result arising from our nonminimal regulator. As such, this result is complete unto itself for any $1 \leq t<\infty$ and requires no further manipulations. That said, we would nevertheless like to broaden the discussion to make contact with a potential field-theoretic interpretation. Toward this end, we shall now make two further adaptations.

Normally, in an EFT, we divide our states into two categories, "light" and "heavy," with respect to some physical scale $\mu$. Within the EFT, those states which are designated light then play a different role relative to those which are designated heavy. However, because the boundary between light and heavy is set in relation to the scale $\mu$, any calculation which distinguishes between these two categories inevitably results in physical quantities (such as Higgs masses) which depend on (or "run" with) the scale $\mu$. In other words, we would expect to obtain a regulated Higgs mass $\hat{m}_{\phi}^{2}(\mu)$ which is a function of the scale $\mu$. Moreover, given that our unregulated Higgs mass $m_{\phi}^{2}$ is only logarithmically divergent prior to regularization, we would expect the regulated quantity $\hat{m}_{\phi}^{2}(\mu)$ to have at most a logarithmic dependence on $\mu$. Indeed, such a relation would be nothing but a renormalization-group equation (RGE) for the Higgs mass.

Our string-theoretic result in Eq. (4.18) already resembles such an RGE. Indeed, as stated above, there are only two adaptations that we must make in order to turn this into an actual RGE. First, we must somehow identify the regulator parameter $t$ which appears in Eq. (4.18) as corresponding to a physical scale $\mu$. Second, we wish to generalize our notion of massless states-states which play a special role in Eq. (4.18) — to states which are simply light with respect to $\mu$.

These two issues are intertwined and have a common resolution. Recall that our string-theoretic calculation, as outlined above, isolated the strictly massless states as special based on the behavior of their potentially divergent contributions as $\tau_{2} \rightarrow \infty$. Indeed, as we have seen, the contributions from states with masses $M>0$ have a built-in Boltzmann-like suppression $\sim e^{-\pi \alpha^{\prime} M^{2} \tau_{2}}$ as $\tau_{2} \rightarrow \infty$, while massless states do not. Thus massless states are unprotected by the Boltzmann suppression factor as $\tau_{2} \rightarrow \infty$, which is why their contributions are subtracted as part of the regularization procedure.

Within the nonminimal regulator, however, we distinguish between two different ranges for $\tau_{2}$ : one range with $1 \leq \tau_{2} \leq t$, and a second range with $t \leq \tau_{2}<\infty$. Only within the second range do we subtract the contributions from the massless states; indeed, massless states are considered "safe" within the first range. But for any finite $t$, it is possible that there are many light states which do not have appreciable Boltzmann suppression factors at $\tau_{2}=t$. Such light (or "effectively massless") states are therefore essentially indistinguishable from truly massless states as far as their Boltzmann suppression factors are concerned. Indeed, it is only as $\tau_{2} \rightarrow \infty$ that we can distinguish the truly massless states relative to all the others.

This suggests that for any finite value of $t$, we can assess whether a given state of mass $M$ is effectively light or heavy according to the magnitude of its corresponding Boltzmann suppression factor at $\tau_{2}=t$ within the partition function. Recalling that the contribution from a physical string state of mass $M$ to the string partition function scales as $e^{-\pi \alpha^{\prime} M^{2} \tau_{2}}$, we can establish an arbitrary criterion for the magnitude of the Boltzmann suppression of a state with mass $M=\mu$ at the cutoff $t$ :

$$
e^{-\pi \alpha^{\prime} \mu^{2} t} \sim e^{-\varepsilon}
$$

where $\varepsilon \geq 0$ is an arbitrarily chosen dimensionless parameter. According to this criterion, states whose Boltzmann factors at $\tau_{2}=t$ exceed $e^{-\varepsilon}$ have not experienced significant Boltzmann suppression and can then be considered light relative to that choice of $t$, while all others can be considered heavy. We thus find that our division between light and heavy states can be demarcated by a running mass scale $\mu(t)$ defined as

$$
\mu^{2}(t) \equiv \frac{\varepsilon}{\pi \alpha^{\prime} t} .
$$

Note, as expected, that $\mu(t) \rightarrow 0$ as $t \rightarrow \infty$. Thus, as expected, the only states that can be considered light as $t \rightarrow \infty$ are those which are exactly massless.

Ultimately, the choice of $\varepsilon$ determines an overall scale for the mapping between $t$ and $\mu$ and is thus a matter of convention. For the sake of simplicity within Eq. (4.20) and our subsequent expressions, we shall henceforth choose $\varepsilon=\pi$, whereupon Eq. (4.20) reduces to

$$
\mu^{2}(t)=\frac{1}{\alpha^{\prime} t} .
$$

With these adaptations, our result for the regulated Higgs mass $\hat{m}_{\phi}^{2}(t)$ in Eq. (4.18) can be rewritten as 


$$
\begin{aligned}
\hat{m}_{\phi}^{2}(\mu)= & \frac{\xi}{4 \pi^{2}} \frac{\Lambda}{\mathcal{M}^{2}}+\frac{1}{2} \mathcal{M}^{2}\left[-\frac{\pi}{3} \operatorname{Str} \mathbb{X}_{1}\right. \\
& \left.+\left(\underset{M \leq \mu}{\operatorname{Str}} \mathbb{X}_{2}\right) \log \left(\frac{\mu^{2}}{\mathcal{M}_{*}^{2}}\right)\right]
\end{aligned}
$$

where we have defined

$$
\mathcal{M}_{*}^{2} \equiv 4 \pi e^{-\gamma} M_{s}^{2}=16 \pi^{3} e^{-\gamma} \mathcal{M}^{2}
$$

and where we have restored the additional universal $\Lambda$-term in Eq. (4.22). We thus see that while the first two terms in Eq. (4.22) are independent of $\mu$ and together constitute what may be considered an overall threshold term, the logarithmic $\mu$-dependence within $\hat{m}_{\phi}^{2}(\mu)$ for any $\mu$ arises from those physical string states which are charged under $\mathbb{X}_{2}$ with masses $M \leq \mu$.

As we have seen, the enhanced nonminimal regulator we are using here operates by explicitly subtracting the contributions of the $\mathbb{X}_{2}$-charged massless states from all regions $\tau_{2} \geq t$. This is a sharp cutoff, and it is natural to wonder how such a cutoff actually maps back onto the strip under the unfolding process. Indeed, answering this question will give us some idea about how this sort of cutoff might be interpreted in field-theoretic language. As expected, imposing a sharp cutoff $\tau_{2} \leq t$ within the $\mathcal{F}$ representation produces both an IR cutoff as well as a UV cutoff on the strip. The IR cutoff is inherited directly from the string-theory cutoff and takes the same form $\tau_{2} \leq t$, thereby excising all parts of the strip with $\tau_{2}$ exceeding $t$, independent of $\tau_{1}$. However, the corresponding UV cutoff is highly nontrivial and is actually sensitive to $\tau_{1}$ as well-a degree of freedom that does not have a direct interpretation in the field theory. Mathematically, this UV cutoff excises from the strip that portion of the region [13]

$$
\underset{(a, c)=1}{\cup} S_{a / c}
$$

which lies within the range $-1 / 2 \leq \tau_{1} \leq 1 / 2$, where $S_{a / c}$ denotes the disk of radius $\left(2 c^{2} t\right)^{-1}$ which is tangent to the $\tau_{2}=0$ axis at $\tau_{1}=a / c$ and where the union in Eq. (4.24) includes all such disks for all relatively prime integers $(a, c)$. Thus, as one approaches the $\tau_{2}=0$ axis of the strip from above, the excised region consists of an infinite series of smaller and smaller disks which are all tangential to this axis in an almost fractal-like pattern. Clearly, all points which actually lie along the $\tau_{2}=0$ axis with $\tau_{1} \in \mathbb{Q}$ are excised for any finite $t$ (and strictly speaking the other points along the $\tau_{2}=0$ axis with $\tau_{1} \notin \mathbb{Q}$ are not even part of the strip). Thus, through this highly unusual UV regulator, all UV divergences on the strip are indeed eliminated for any finite $t$. Of course, this excised UV region is nothing but the image of the IR-excised region $\tau_{2} \geq t$ under all of the modular transformations (namely those within the coset $\Gamma_{\infty} \backslash \Gamma$ ) that play a role in building the strip from $\mathcal{F}$. However, in field-theoretic language this amounts to a highly unusual UV regulator indeed.

\section{E. Results using the modular-invariant regulator}

Finally, we turn to the results for the Higgs mass that are obtained using the fully modular-invariant regulator $\hat{\mathcal{G}}_{\rho}\left(a, \tau_{2}\right)$ in Eq. (3.39). As we have stressed, only such results can be viewed as faithful to the modular symmetry that underlies closed-string theory, and therefore only such results can be viewed as truly emerging from closed-string theories.

We have seen in Eq. (4.4) that the string-theoretic Higgs mass $m_{\phi}^{2}$ has two contributions: one of these stems from the $\mathcal{X}_{i}$ insertions and requires regularization, while the othernamely the cosmological-constant term-is finite within any tachyon-free modular-invariant theory and hence does not. When discussing the possible regularizations of the Higgs mass using the minimal and nonminimal regulators in Secs. IV C and IV D, we simply carried the cosmological-constant term along within our calculations and focused on applying our regulators to the contributions with $\mathcal{X}_{i}$ insertions. This was adequate for the minimal and nonminimal regulators because these regulators involve the explicit subtraction of divergences and thus have no effect on quantities which are already finite and therefore lack divergences to be subtracted. Our modular-invariant regulator, by contrast, operates by deforming the theory. Indeed, this deformation has the effect of multiplying the partition function of the theory by a new factor $\hat{\mathcal{G}}_{\rho}(a, \tau)$. As such, this regularization procedure can be expected to have an effect even when acting on finite quantities such as $\Lambda$. When regularizing the Higgs mass in this manner, we must therefore consider how this regulator affects both classes of Higgs-mass contributions-those involving nontrivial $\mathcal{X}_{i}$ insertions, and those coming from the cosmological constant. Indeed, with $\left.\hat{m}_{\phi}^{2}(\rho, a)\right|_{\mathcal{X}, \Lambda}$ respectively denoting these two classes of contributions to the $\hat{\mathcal{G}}$-regulated version $\hat{m}_{\phi}^{2}(\rho, a)$ of the otherwise-divergent string-theoretic Higgs mass in Eq. (4.4), we can write

$$
\begin{aligned}
\hat{m}_{\phi}^{2}(\rho, a) & \left.\equiv \hat{m}_{\phi}^{2}(\rho, a)\right|_{\mathcal{X}}+\left.\hat{m}_{\phi}^{2}(\rho, a)\right|_{\Lambda} \\
& \left.\equiv \hat{m}_{\phi}^{2}(\rho, a)\right|_{\mathcal{X}}+\frac{\xi}{4 \pi^{2} \mathcal{M}^{2}} \hat{\Lambda}(\rho, a)
\end{aligned}
$$

We shall now consider each of these contributions in turn.

\section{Contributions from terms with charge insertions}

Our first contribution in Eq. (4.25) is given by

$$
\left.\hat{m}_{\phi}^{2}(\rho, a)\right|_{\mathcal{X}} \equiv-\frac{\mathcal{M}^{2}}{2}\left\langle\mathcal{X}_{1}+\mathcal{X}_{2}\right\rangle_{\mathcal{G}}
$$


where

$$
\langle A\rangle_{\mathcal{G}} \equiv \int_{\mathcal{F}} \frac{d^{2} \tau}{\tau_{2}^{2}}\left\{\left[\tau_{2}^{-1} \sum_{m, n}(-1)^{F} A \bar{q}^{m} q^{n}\right] \hat{\mathcal{G}}_{\rho}(a, \tau)\right\}
$$

with $m \equiv \alpha^{\prime} M_{R}^{2} / 4, n \equiv \alpha^{\prime} M_{L}^{2} / 4$. Indeed, the insertion of $\hat{\mathcal{G}}_{\rho}(a, \tau)$ into the integrand of Eq. (4.27) is what tames the logarithmic divergence. Following the result in Eq. (3.35) we then find that $\hat{m}_{\phi}^{2}(\rho, a)$ can be expressed as

$$
\left.\tilde{m}_{\phi}^{2}(\rho, a)\right|_{\mathcal{X}}=\frac{\pi}{3} \operatorname{Res}_{s=1} \int_{0}^{\infty} d \tau_{2} \tau_{2}^{s-2} \hat{g}_{\rho}\left(a, \tau_{2}\right)
$$

where

$$
\begin{aligned}
\hat{g}_{\rho}\left(a, \tau_{2}\right) \equiv & -\frac{\mathcal{M}^{2}}{2} \int_{-1 / 2}^{1 / 2} d \tau_{1} \\
& \times\left\{\left[\sum_{m, n}(-1)^{F}\left(\mathbb{X}_{1}+\tau_{2} \mathbb{X}_{2}\right) \bar{q}^{m} q^{n}\right] \hat{\mathcal{G}}_{\rho}(a, \tau)\right\} \\
\approx & -\frac{\mathcal{M}^{2}}{2}\left[\operatorname{Str}\left(\mathbb{X}_{1}+\tau_{2} \mathbb{X}_{2}\right) e^{-\pi \alpha^{\prime} M^{2} \tau_{2}}\right] \hat{\mathcal{G}}_{\rho}\left(a, \tau_{2}\right) .
\end{aligned}
$$

Note that in passing to the approximate factorized form in the final expression of Eq. (4.29), we have followed the result in Eq. (3.37) and explicitly restricted our attention to those cases with $a \ll 1$, as appropriate for the regulator function $\hat{\mathcal{G}}_{\rho}(a, \tau)$. Indeed, the term within square brackets in the third line of Eq. (4.51) is our desired supertrace over physical string states, while the regulator function $\hat{\mathcal{G}}_{\rho}\left(a, \tau_{2}\right)$-an example of which is plotted in the right panel of Fig. 1-generally eliminates the divergence that would otherwise have arisen as $\tau_{2} \rightarrow \infty$ for any $a>0$. Moreover, we learn that as a consequence of the identity in Eq. (3.38)—an identity which holds for $\hat{\mathcal{G}}$ as well as for $\mathcal{G}$ itself - the behavior shown in the right panel of Fig. 1 can be symmetrically "reflected" through $\tau_{2}=1$, resulting in the same suppression behavior as $\tau_{2} \rightarrow 0$.

The next step is to substitute Eq. (4.29) back into Eq. (4.28) and evaluate the residue at $s=1$. In general, the presence of the regulator function $\hat{\mathcal{G}}_{\rho}\left(a, \tau_{2}\right)$ within Eq. (4.29) renders this calculation somewhat intricate. However, we know that $\hat{\mathcal{G}}_{\rho}\left(a, \tau_{2}\right) \rightarrow 1$ as $a \rightarrow 0$. Indeed, having already exploited our regulator in allowing us to pass from Eq. (4.26) to Eq. (4.28), we see that taking $a \rightarrow 0$ corresponds to the limit in which we subsequently remove our regulator. Let us first focus on the contributions from massive states. In the $a \rightarrow 0$ limit, we then obtain

$$
\begin{aligned}
\operatorname{Res}_{s=1} & \int_{0}^{\infty} d \tau_{2} \tau_{2}^{s-2} \hat{g}_{\rho}\left(a, \tau_{2}\right) \\
= & -\frac{1}{2} \mathcal{M}^{2} \operatorname{Res}_{s=1}\left[\Gamma(s-1) \underset{M>0}{\operatorname{Str}} \mathbb{X}_{1}\left(\pi \alpha^{\prime} M^{2}\right)^{1-s}\right. \\
& +\Gamma(s) \underset{M>0}{\left.\operatorname{Str} \mathbb{X}_{2}\left(\pi \alpha^{\prime} M^{2}\right)^{-s}\right]} \\
= & -\frac{1}{2} \mathcal{M}^{2} \underset{M>0}{\operatorname{Str} \mathbb{X}_{1}}
\end{aligned}
$$

whereupon we find that the contribution from massive states yields

$M>0:\left.\quad \lim _{a \rightarrow 0} \hat{m}_{\phi}^{2}(\rho, a)\right|_{\mathcal{X}}=-\frac{\pi}{6} \mathcal{M}^{2} \underset{M>0}{\operatorname{Str}} \mathbb{X}_{1}$.

This result is independent of $\rho$. Moreover, as expected for massive states, this contribution is finite. Of course, there will also be contributions from massless states. In general, these contributions are more subtle to evaluate, and we know that as $a \rightarrow 0$ the effective removal of the regulator will lead to divergences coming from potentially nonzero values of $\underset{M=0}{\operatorname{Str}} \mathbb{X}_{2}$ (since it is the massless states which are charged under $\mathbb{X}_{2}$ which cause the Higgs mass to diverge). However, massless states charged under $\mathbb{X}_{1}$-like the massive states-do not lead to divergences. We might therefore imagine restricting our attention to cases with $\underset{M=0}{\operatorname{Str}} \mathbb{X}_{2}=0$, and deforming our theory slightly so that these massless $\mathbb{X}_{1}$-charged states accrue small nonzero masses. In that case, the calculation in Eq. (4.30) continues to apply. We can imagine removing this deformation without encountering any divergences. This suggests that the full result for the regulated Higgs mass in the $a \rightarrow 0$ limit should be the same as in Eq. (4.31), but with massless $\mathbb{X}_{1}$-charged states also included. We therefore expect

$$
\left.\lim _{a \rightarrow 0} \hat{m}_{\phi}^{2}(\rho, a)\right|_{\mathcal{X}}=-\frac{\pi}{6} \mathcal{M}^{2} \operatorname{Str} \mathbb{X}_{1}
$$

in cases for which $\underset{M=0}{\operatorname{Str}} \mathbb{X}_{2}=0$. We shall rigorously confirm this result below.

As discussed in Sec. III B 3, the two quantities $(\rho, a)$ that parametrize our modular-invariant regulator are analogous to the quantity $t$ that parametrized our nonminimal regulator. Indeed, these quantities effectively specify the value of the "cutoff" imposed by these regulators, and as such we can view these quantities as corresponding to a floating physical mass scale $\mu$. This scale $\mu$ is defined in terms of $t$ for the nonminimal regulator in Eq. (4.21), and we have already seen that maintaining alignment between this regulator and our modular-invariant regulator requires that we enforce the condition in Eq. (3.44). We shall therefore identify a physical scale $\mu$ for our modular-invariant regulator as 


$$
\mu^{2}(\rho, a) \equiv \frac{\rho a^{2}}{\alpha^{\prime}} .
$$

Since $\rho \sim \mathcal{O}(1)$, the $a \ll 1$ region for our regulator corresponds to the restricted region $\mu \ll M_{s}$.

The identification in Eq. (4.33) enables us to rewrite our result in Eq. (4.32) in the more suggestive form

$$
\left.\lim _{\mu \rightarrow 0} \hat{m}_{\phi}^{2}(\mu)\right|_{\mathcal{X}}=-\frac{\pi}{6} \mathcal{M}^{2} \operatorname{Str} \mathbb{X}_{1}
$$

in cases for which $\underset{M=0}{\operatorname{Str}} \mathbb{X}_{2}=0$. In EFT language, we can therefore regard this result as holding in the deep infrared.
The natural question that arises, then, is to determine how our regulated Higgs mass $\hat{m}_{\phi}^{2}(\mu)$ runs as a function of the scale $\mu$. In order to do this, we need to evaluate $\hat{m}_{\phi}^{2}(\rho, a)$ as a function of $a$ for small $a \ll 1$ without taking the full $a \rightarrow 0$ limit.

As indicated above, this calculation is somewhat intricate and is presented in Appendix A. The end result, given in Eq. (A16), is an expression for $\hat{m}_{\phi}^{2}(\rho, a)$ which is both exact and valid for all $a$. Using the identification in Eq. (4.33) and henceforth taking the benchmark value $\rho=2$, the result in Eq. (A16) can then be expressed in terms of the scale $\mu$, yielding

$$
\begin{aligned}
\left.\hat{m}_{\phi}^{2}(\mu)\right|_{\mathcal{X}}= & \frac{\mathcal{M}^{2}}{1+\mu^{2} / M_{s}^{2}}\left\{\underset{M=0}{\operatorname{Str} \mathbb{X}_{1}}\left[-\frac{\pi}{6}\left(1+\mu^{2} / M_{s}^{2}\right)\right]+\underset{M=0}{\operatorname{Str} \mathbb{X}_{2}}\left[\log \left(\frac{\mu}{2 \sqrt{2} e M_{s}}\right)\right]\right. \\
& +\underset{M>0}{\operatorname{Str}} \mathbb{X}_{1}\left\{-\frac{\pi}{6}-\frac{1}{2 \pi}\left(\frac{M}{\mathcal{M}}\right)^{2}\left[\mathcal{K}_{0}^{(0,1)}\left(\frac{2 \sqrt{2} \pi M}{\mu}\right)+\mathcal{K}_{2}^{(0,1)}\left(\frac{2 \sqrt{2} \pi M}{\mu}\right)\right]\right\} \\
& \left.+\underset{M>0}{\operatorname{Str} \mathbb{X}_{2}}\left[2 \mathcal{K}_{0}^{(0,1)}\left(\frac{2 \sqrt{2} \pi M}{\mu}\right)-\mathcal{K}_{1}^{(1,2)}\left(\frac{2 \sqrt{2} \pi M}{\mu}\right)\right]\right\}
\end{aligned}
$$

where we have defined the Bessel-function combinations

$$
\mathcal{K}_{\nu}^{(n, p)}(z) \equiv \sum_{r=1}^{\infty}(r z)^{n}\left[K_{\nu}(r z / \rho)-\rho^{p} K_{\nu}(r z)\right]
$$

with $K_{\nu}(z)$ denoting the modified Bessel function of the second kind. We see, then, that the contributions to the running of $\left.\hat{m}_{\phi}^{2}(\mu)\right|_{\mathcal{X}}$ from the different states in our theory depend rather nontrivially on their masses and on their various $\mathbb{X}_{1}$ and $\mathbb{X}_{2}$ charges, with the contributions from each string state with nonzero mass $M$ governed by various combinations of Bessel functions $K_{\nu}(z)$ with arguments $z \sim M / \mu$.

There is a plethora of physics wrapped within Eq. (4.35), and we shall unpack this result in several stages. First, it is straightforward to take the $\mu \rightarrow 0$ limit of Eq. (4.35) in order to verify our expectation in Eq. (4.32). Indeed, in the $\mu \rightarrow 0$ limit, we have $z \rightarrow \infty$ for all $M>0$. Since

$$
\mathcal{K}_{\nu}^{(n, p)}(z) \sim \sqrt{\frac{\pi \rho}{2}} z^{n-1 / 2} e^{-z / \rho} \quad \text { as } z \rightarrow \infty
$$

it then follows that all of the terms involving Bessel functions in Eq. (4.35) vanish exponentially in the $\mu \rightarrow 0$ limit. For cases in which $\underset{M=0}{\operatorname{Str}} \mathbb{X}_{2}=0$ [i.e., cases in which the original Higgs mass $m_{\phi}^{2}$ is finite, with no massless states charged under $\mathbb{X}_{2}$ ], we thus reproduce the result in Eq. (4.32).
Using the result in Eq. (4.35), we can also study the running of $\hat{m}_{\phi}^{2}(\mu)$ as a function of $\mu>0$. Of course, given that our $\hat{\mathcal{G}}$ function acts as a regulator only for $a \ll 1$, our analysis is restricted to the $\mu \ll M_{s}$ region. Let us first concentrate on the contributions from the terms within Eq. (4.35) that do not involve Bessel functions. These contributions are given by

$$
\mathcal{M}^{2}\left\{-\frac{\pi}{6} \operatorname{Str} \mathbb{X}_{1}+\underset{M=0}{\operatorname{Str}} \mathbb{X}_{2} \log \left(\frac{\mu}{2 \sqrt{2} e M_{S}}\right)\right\} .
$$

From this we see that our deep-infrared contribution to $\hat{m}_{\phi}^{2}$ in Eq. (4.32) actually persists as an essentially constant contribution for all scales $\mu \ll M_{s}$. We also see from Eq. (4.38) that each massless string state also contributes an additional logarithmic running which is proportional to its $\mathbb{X}_{2}$ charge and which persists all the way into the deep infrared. Given that massless $\mathbb{X}_{2}$-charged states are precisely the states that led to the original logarithmic divergence in the unregulated Higgs mass $m_{\phi}^{2}$, this logarithmic running is completely expected. Indeed, it formally leads to a divergence in our regulated Higgs mass $\hat{m}_{\phi}^{2}(\mu)$ in the full $\mu \rightarrow 0$ limit (at which our regulator is effectively removed), but otherwise produces a finite contribution for all other $\mu>0$. The issues connected with this logarithm are actually no different from those that arise in an ordinary field-theoretic calculation. We shall discuss these issues in more detail in Sec. VI but in the meantime this term will not concern us further. 
The remaining contributions are those arising from the terms within Eq. (4.35) involving supertraces over Bessel functions. Although our analysis is restricted to the $\mu \ll M_{s}$ region, our supertraces receive contributions from the entire string spectrum. This necessarily includes states with masses $M \gtrsim M_{s}$, but may also include potentially light states with nonzero masses far below $M_{s}$. The existence of such light states depends on our string construction and on the specific string model in question. Indeed, such states are particularly relevant for the kinds of string models that motivate our analysis, namely (nonsupersymmetric) string models in which the Standard Model is realized directly within the low-energy spectrum.

The Bessel functions corresponding to states with masses $M \gtrsim M_{s}$ have arguments $z \sim M / \mu \gg 1$ when $\mu \ll M_{s}$. As a result, in accordance with Eqs. (4.35) and (4.37), the contributions from these states to the running of $\left.\hat{m}_{\phi}^{2}(\mu)\right|_{\mathcal{X}}$ are exponentially suppressed. It then follows that the dominant contributions to the Bessel-function running of $\hat{m}_{\phi}(\mu)$ within the $\mu \ll M_{s}$ region come from the correspondingly light states, i.e., states with masses $M \ll M_{s}$. However, for states with masses $M \ll M_{s}$, we see from Eq. (4.35) that the corresponding Bessel-function contributions which are proportional to their $\mathbb{X}_{1}$ charges are all suppressed by a factor $(M / \mathcal{M})^{2}$. We thus conclude that the contribution from a state of nonzero mass $M \ll M_{s}$ within the string spectrum is sizable only when this state carries a nonzero $\mathbb{X}_{2}$ charge. Indeed, we see from Eq. (4.35) that this contribution for each bosonic degree of freedom of mass $M$ is given by

$$
2 \mathcal{K}_{0}^{(0,1)}(z)-\mathcal{K}_{1}^{(1,2)}(z)
$$

per unit of $\mathbb{X}_{2}$ charge, where $z \equiv 2 \sqrt{2} \pi M / \mu$.

In Fig. 2, we plot this contribution as a function of $\mu / M$. As expected, we see that states with $M \gg \mu$ produce no running and can be ignored-essentially they have been "integrated out" of our theory at the scale $\mu$ and leave behind only an exponential tail. By contrast, states with $M \lesssim \mu$ are still dynamical at the scale $\mu$. We see from Fig. 2 that their effective contributions are then effectively logarithmic. Indeed, as $z \rightarrow 0$, one can show that [28]

$$
\begin{aligned}
& \mathcal{K}_{0}^{(0,1)}(z) \sim-\frac{1}{2} \log z+\frac{1}{2}[\log (2 \pi)-\gamma], \\
& \mathcal{K}_{1}^{(1,2)}(z) \sim 1
\end{aligned}
$$

where $\gamma$ is the Euler-Mascheroni constant. This leads to an asymptotic logarithmic running of the form

$$
\log \left[\frac{1}{\sqrt{2}} e^{-(\gamma+1)} \frac{\mu}{M}\right]
$$

for $\mu \gg M$ in Fig. 2. Finally, between these two behaviors, we see that the expression in Eq. (4.39) interpolates

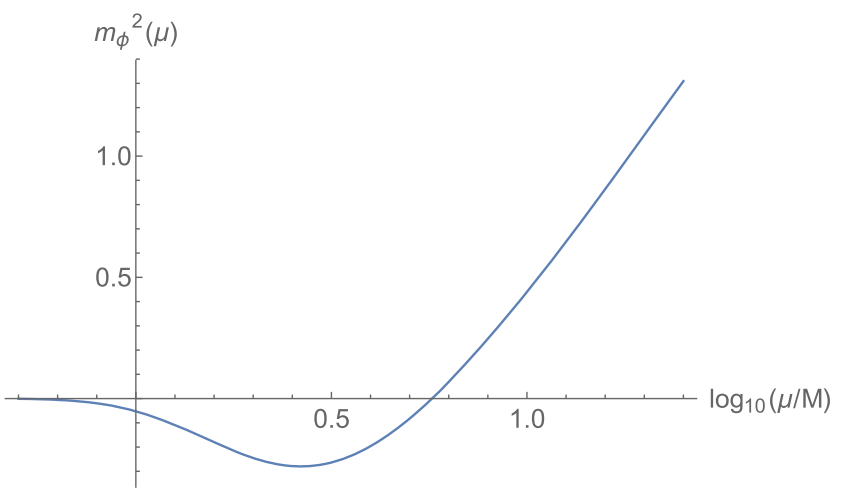

FIG. 2. The expression in Eq. (4.39), plotted as a function of $\mu / M$. This quantity is the Bessel-function contribution per unit $\mathbb{X}_{2}$ charge to the running of the regulated Higgs mass $\left.\hat{m}_{\phi}^{2}(\mu)\right|_{\mathcal{X}} / \mathcal{M}^{2}$ from a bosonic state of nonzero mass $M$. This contribution is universal for all $\mu / M$ and assumes only that $\mu \ll M_{s}$. When $\mu \gg M$, the state is fully dynamical and produces a running which is effectively logarithmic. By contrast, when $\mu \ll M$, the state is heavier than the scale $\mu$ and is effectively integrated out, thereby suppressing any contributions to the running. Finally, within the intermediate $\mu \approx M$ region, the Bessel-function expression in Eq. (4.39) provides a smooth connection between these two asymptotic behaviors and even gives rise to a transient "dip" in the overall running. Note that for a fixed scale $\mu$, adjusting the mass $M$ of the relevant state upward or downward simply corresponds to shifting this curve rigidly to the right or left, respectively. In this way one can imagine summing over all such contributions to the running as one takes the supertrace over the entire $\mathbb{X}_{2}$-charged string spectrum.

smoothly and even gives rise to a transient "dip." This is a uniquely string-theoretic behavior resulting from the specific combination of Bessel functions in Eq. (4.39). Of course, the statistics factor $(-1)^{F}$ within the supertrace flips the sign of this contribution for degrees of freedom which are fermionic.

Thus far we have focused on the Higgs-mass running, as shown in Fig. 2, from a single massive string degree of freedom of mass $M$. However, the contribution from another string state with a different mass $M^{\prime}$ can be simply obtained by rigidly sliding this curve toward the left or right (respectively corresponding to cases with $M^{\prime}<M$ and $M^{\prime}>M$, respectively). The complete supertrace contribution in Eq. (4.35) is then obtained by summing over all of these curves, each with its appropriate horizontal displacement and each weighted by the corresponding net (bosonic minus fermionic) number of degrees of freedom. The resulting net running from the final term within Eq. (4.35) is therefore highly sensitive to the properties of the massive $\mathbb{X}_{2}$-charged part of the string spectrum. This will be discussed further in Sec. IVE3. Of course, as discussed above, the contributions from states with $M^{\prime} \gg \mu$ are exponentially suppressed. Thus, for any $\mu$, the only states which contribute meaningfully to this Besselfunction running of the Higgs mass are those with $M \lesssim \mu$. 
Thus, combining these Bessel-function contributions with those from Eq. (4.38) and keeping only those (leading) terms which dominate when $M \ll \mu \ll M_{s}$, we see that we can approximate the exact result in Eq. (4.35) as

$$
\begin{aligned}
\left.\hat{m}_{\phi}^{2}(\mu)\right|_{\mathcal{X}} \approx & -\frac{\pi}{6} \mathcal{M}^{2} \operatorname{Str} \mathbb{X}_{1}+\mathcal{M}^{2} \operatorname{Str}_{M=0} \mathbb{X}_{2} \log \left(\frac{\mu}{2 \sqrt{2} e M_{s}}\right) \\
& +\mathcal{M}^{2} \underset{0<M \lesssim \mu}{\operatorname{Str}} \mathbb{X}_{2} \log \left[\frac{1}{\sqrt{2}} e^{-(\gamma+1)} \frac{\mu}{M}\right] .
\end{aligned}
$$

Interestingly, we see that to leading order, the $\mathbb{X}_{1}$ charges of the string states only contribute to an overall constant term in Eq. (4.42), and they do this for all states regardless of their masses. By contrast, it is the $\mathbb{X}_{2}$ charges of the states which induce a corresponding running, and this only occurs for those states within the EFT at the scale $\mu$-i.e., those light states with masses $M \lesssim \mu$.

The net running produced by the final term in Eq. (4.42) can exhibit a variety of behaviors. To understand this, let us consider the behavior of this term as we increase $\mu$ from the deep infrared. Of course, this term does not produce any running at all until we reach $\mu \sim M_{\text {lightest }}$, where $M_{\text {lightest }}$ is the mass of the lightest massive string state carrying a nonzero $\mathbb{X}_{2}$ charge. This state then contributes a logarithmic running which persists for all higher $\mu$. However, as $\mu$ increases still further, additional $\mathbb{X}_{2}$-charged string states enter the EFT and contribute their own individual logarithmic contributions. Of course, if these additional states have masses $M \gg M_{\text {lightest }}$, the logarithmic nature of the running shown in Fig. 2 from the state with mass $M_{\text {lightest }}$ will survive intact until $\mu \sim M$. However, if the spectrum of states is relatively dense beyond $M_{\text {lightest }}$, the logarithmic contributions from each of these states must be added together, leading to a far richer behavior.

One important set of string models exhibiting the latter property are those involving a relatively large compactification radius $R$. In such cases, we can identify $M_{\text {lightest }} \sim 1 / R$, whereupon we expect an entire tower of corresponding $\mathrm{KK}$ states of masses $M_{k} \sim k / R, k \in \mathbb{Z}^{+}$, each sharing a common charge $\mathbb{X}_{2}$ and a common degeneracy of states $g$. For any scale $\mu$, the final term in Eq. (4.42) then takes the form

$$
\begin{aligned}
\mathcal{M}^{2} g \mathbb{X}_{2} \sum_{k=1}^{\mu R} \log \left[\frac{1}{\sqrt{2}} e^{-(\gamma+1)} \frac{\mu R}{k}\right] \\
=\mathcal{M}^{2} g \mathbb{X}_{2}\left\{\mu R \log \left[\frac{1}{\sqrt{2}} e^{-(\gamma+1)} \mu R\right]-\log (\mu R) !\right\} \\
=\mathcal{M}^{2} g \mathbb{X}_{2}\left\{\log \left[\frac{1}{\sqrt{2}} e^{-(\gamma+1)}\right]+1\right\} \mu R
\end{aligned}
$$

where in passing to the third line we have used Stirling's approximation $\log N ! \approx N \log N-N$. We thus see that in such cases our sum over logarithms actually produces a power-law running. In this case the running is linear, but in general the KK states associated with $d$ large toroidally compactified dimensions collectively yield a regulated Higgs mass whose running scales as $\mu^{d}$.

This phenomenon whereby a sum over KK states deforms a running from logarithmic to power law is well known from phenomenological studies of theories with large extra dimensions, where it often plays a crucial role (see, e.g., Refs. [29-31]). This phenomenon can ultimately be understood from the observation that a large compactification radius effectively increases the overall spacetime dimensionality of the theory, thereby shifting the mass dimensions of quantities such as gauge couplings and Higgs masses and simultaneously shifting their corresponding runnings. Indeed, as discussed in detail in Appendices A and B of Ref. [30] (and as illustrated in Fig. 11 therein), the emergence of power-law running from logarithmic running is surprisingly robust.

Of course, it may happen that the spectrum of light states not only has a lightest mass $M_{\text {lightest }}$ but also a heaviest mass $M_{\text {heaviest }}$, with a significant mass gap beyond this before reaching even heavier scales. If such a situation were to arise (but clearly does not within the large extra-dimension scenario described above), then the corresponding running of $\left.\hat{m}_{\phi}^{2}(\mu)\right|_{\mathcal{X}}$ would only be power law within the range $M_{\text {lightest }} \lesssim \mu \lesssim M_{\text {heaviest }}$. For $\mu>M_{\text {heaviest }}$, by contrast, the running would then revert back to logarithmic.

In summary, we see that while the first term within Eq. (4.42) represents an overall constant contribution arising from the entire spectrum of $\mathbb{X}_{1}$-charged states, the second term represents an overall logarithmic contribution from precisely the massless $\mathbb{X}_{2}$-charged states which were the source of the original divergence of the unregulated Higgs mass $m_{\phi}^{2}$. By contrast, the final term in Eq. (4.42) represents the nontrivial contribution to the running from the massive $\mathbb{X}_{2}$-charged states. As we have seen, the latter contribution can exhibit a variety of behaviors, ranging from logarithmic (in cases with relatively large mass splittings between the lightest massive $\mathbb{X}_{2}$-charged states) to power law (in cases with relatively small uniform mass splittings between such states). Of course, depending on the details of the underlying string spectrum, mixtures between these different behaviors are also possible.

\section{Contribution from the cosmological constant}

Let us now turn to the second term in Eq. (4.25). This contribution lacks $\mathcal{X}_{i}$ insertions and arises from the cosmological-constant term in Eq. (4.4). Although this contribution is the result of a universal shift in the background moduli and is thus independent of the specific $\mathcal{T}$-matrices, we shall now demonstrate that it too can be expressed as a supertrace over the physical string spectrum. 
It also develops a scale dependence when subjected to our modular-invariant regulator.

Within the definition of $\Lambda$ in Eq. (2.54), the integrand function $\mathcal{F}(\tau, \bar{\tau})$ is simply $\left(-\mathcal{M}^{4} / 2\right) \mathcal{Z}(\tau, \bar{\tau})$ where $\mathcal{Z}(\tau, \bar{\tau})$ is the partition function of the string in the Higgsed phase. Of course, if this theory exhibits unbroken spacetime supersymmetry, the contributions from the bosonic states in the spectrum cancel level by level against those from their fermionic superpartners. In such cases we then have $\mathcal{Z}=0$, implying $\Lambda=0$. Otherwise, for heterotic strings, we necessarily have $\mathcal{Z} \neq 0$. Indeed, it is a theorem (first introduced in Ref. [9] and discussed more recently, e.g., in Ref. [32]) that any nonsupersymmetric heterotic-string model in $D$ spacetime dimensions must contain an off-shell tachyonic proto-graviton state whose contribution to the partition function remains uncanceled. This then results in a string partition function whose power-series expansion has the leading behavior $Z=(D-2) / q+\cdots$.

In principle this proto-graviton contribution would appear to introduce an exponential divergence as $\tau_{2} \rightarrow \infty$, thereby taking us beyond the realm of validity for the mathematical techniques presented in Sec. III A. However, this tachyonic state is off-shell and thus does not appear in the actual physical string spectrum. Indeed, as long as there are no additional on-shell tachyons present in the theory, the corresponding integral $\Lambda$ is fully convergent because the integral over the fundamental domain $\mathcal{F}$ comes with an explicit instruction that we are to integrate across $\tau_{1}$ in the $\tau_{2}>1$ region of $\mathcal{F}$ before integrating over $\tau_{2}$. This integration therefore prevents the proto-graviton state from contributing to $\Lambda$ within the $\tau_{2}>1$ region of integration, and likewise prevents this state from contributing to $g\left(\tau_{2}\right)$.

Assuming, therefore, that we can disregard the protograviton contribution to $\mathcal{Z}$ as $\tau_{2} \rightarrow \infty$, we find that $\mathcal{Z} \sim \tau_{2}^{-1}$ as $\tau_{2} \rightarrow \infty$. Thus $\mathcal{Z}$ is effectively of rapid decay and we can use the original Rankin-Selberg results in Eq. (3.12). In this connection, we note that this assumption regarding the proto-graviton contribution finds additional independent support through the arguments presented in Ref. [16] which demonstrate that any contributions from the proto-graviton beyond those in Eq. (3.12) are suppressed by an infinite volume factor in all spacetime dimensions $D>2$. A similar result is also true in string models with exponentially suppressed cosmological constants [32].

With $\mathcal{Z}$ taking the form in Eq. (3.2) and with the mass $M$ of each physical string state identified via $\alpha^{\prime} M^{2}=$ $2(m+n)=4 m$, we then have

$$
g\left(\tau_{2}\right)=-\frac{\mathcal{M}^{4}}{2} \tau_{2}^{-1} \operatorname{Str} e^{-\pi \alpha^{\prime} M^{2} \tau_{2}}
$$

Inserting this result into Eq. (3.12) and performing the $\tau_{2}$ integral then yields [18]

$$
\begin{aligned}
\Lambda & =-\frac{\mathcal{M}^{4}}{2} \frac{\pi}{3} \operatorname{Res}_{s=1}\left[\pi^{2-s} \Gamma(s-2) \operatorname{Str}\left(\alpha^{\prime} M^{2}\right)^{2-s}\right] \\
& =\frac{\mathcal{M}^{4}}{2} \frac{\pi^{2}}{3} \operatorname{Str}\left(\alpha^{\prime} M^{2}\right) \\
& =\frac{1}{24} \mathcal{M}^{2} \operatorname{Str} M^{2} .
\end{aligned}
$$

We thus see that $\Lambda$ is given as a universal supertrace over all physical string states, and not only those with specific charges relative to the Higgs field.

As evident from the form of the final supertrace in Eq. (4.45), massless states do not ultimately contribute within this expression for $\Lambda$. Strictly speaking, our derivation in Eq. (4.45) already implicitly assumed this, given that the intermediate steps in Eq. (4.45) are valid only for $M>0$. However, it is easy to see that the contributions from massless states lead to a $\tau_{2}$-integral whose divergence has no residue at $s=1$. Thus, massless states make no contribution to this expression, and the result in Eq. (4.45) stands.

This does not mean that massless states do not contribute to $\Lambda$, however. Rather, this just means that the constraints from modular invariance so tightly connect the contributions to $\Lambda$ from the massless states to those from the massive states (and also those from the unphysical string states of any mass) that an expression for $\Lambda$ as in Eq. (4.45) becomes possible.

For further insight into this issue, it is instructive to obtain this same result through Eq. (3.14). We then have

$$
\Lambda=-\frac{\pi}{3} \frac{\mathcal{M}^{4}}{2} \lim _{\tau_{2} \rightarrow 0}\left[\tau_{2}^{-1} \operatorname{Str} \exp \left(-\pi \alpha^{\prime} M^{2} \tau_{2}\right)\right]
$$

Expanding the exponential $e^{-x} \approx 1-x+\cdots$ and taking the $\tau_{2} \rightarrow 0$ limit of each term separately, we find that the linear term leads directly to the result in Eq. (4.45) while the contributions from all of the higher terms vanish. Interestingly, the constant term would a priori appear to lead to a divergence for $\Lambda$. The fact that $\Lambda$ is finite in such theories then additionally tells us that [18]

$$
\operatorname{Str} \mathbf{1}=0
$$

As apparent from our derivation, this constraint must hold for any tachyon-free modular-invariant theory (i.e., any modular-invariant theory in which $\Lambda$ is finite). Indeed, this is one of the additional constraints from modular invariance which relates the contributions of the physical string states which are massless to the contributions of those which are massive. Thus, we may regard the result in Eq. (4.45) like all of the results of this paper-as holding within a modular-invariant context in which other constraints such as that in Eq. (4.47) are also simultaneously satisfied. We also see from this analysis that our supertrace definition in Eq. (4.10) may be more formally defined as [18] 


$$
\operatorname{Str} A \equiv \lim _{y \rightarrow 0} \sum_{\text {physical } i}(-1)^{F_{i}} A_{i} e^{-y \alpha^{\prime} M_{i}^{2}}
$$

The supertrace results in Eqs. (4.45) and (4.47) were first derived in Ref. [18]. As discussed in Refs. [18,19], these results hold for all tachyon-free heterotic strings in four dimensions, and in fact similar results hold in all spacetime dimensions $D>2$. For theories exhibiting spacetime supersymmetry, these relations are satisfied rather trivially. However, even if the spacetime supersymmetry is brokenand even if the scale of supersymmetry breaking is relatively large or at the Planck scale-these results nevertheless continue to hold. In such cases, these supertrace relations do not arise as the results of pairwise cancellations between the contributions of bosonic and fermionic string states. Rather, these relations emerge as the results of conspiracies that occur across the entire string spectrum, with the bosonic and fermionic string states always carefully arranging themselves at all string mass levels so as to exhibit a socalled "misaligned supersymmetry" [17,19]. No pairing of bosonic and fermionic states occurs within misaligned supersymmetry, yet misaligned supersymmetry ensures that these supertrace relations are always satisfied. These results therefore constrain the extent to which supersymmetry can be broken in tachyon-free string theories while remaining consistent with modular invariance.

The results that we have obtained thus far pertain to the cosmological constant $\Lambda$. As such, they would be sufficient if we were aiming to understand this quantity unto itself, since $\Lambda$ is finite in any tachyon-free modular-invariant theory and hence requires no regulator. However, in this paper our interest in this quantity stems from the fact that $\Lambda$ is an intrinsic contributor to the total Higgs mass in Eq. (2.55), and we already have seen that the Higgs mass requires regularization. At first glance, one might imagine regulating the terms with nonzero $\mathcal{X}_{i}$ insertions while leaving the $\Lambda$-term alone. However, it is ultimately inappropriate to regularize only a subset of terms that contribute to the Higgs mass-for consistency we must apply the same regulator to the entire expression at once. Indeed, we recall from Sec. II that the entire Higgs-mass expression including $\Lambda$ forms a modularinvariant unit, with $\Lambda$ emerging from the modular completion of some of the terms with nontrivial $\mathcal{X}_{i}$ insertions. For this reason, we shall now study the analogously regulated cosmological constant

$$
\hat{\Lambda}(\rho, a) \equiv \int_{\mathcal{F}} \frac{d^{2} \tau}{\tau_{2}^{2}} \mathcal{Z}(\tau) \hat{\mathcal{G}}_{\rho}(a, \tau)
$$

and determine the extent to which this regularized cosmological constant can also be expressed in terms of supertraces over the physical string states.

Our discussion proceeds precisely as for the terms involving the $\mathcal{X}_{i}$ insertions. Following the result in Eq. (3.35) we find that $\hat{\Lambda}(\rho, a)$ can be expressed as

$$
\hat{\Lambda}(\rho, a)=\frac{\pi}{3} \operatorname{Res}_{s=1} \int_{0}^{\infty} d \tau_{2} \tau_{2}^{s-3} \hat{g}_{\rho}\left(a, \tau_{2}\right)
$$

where

$$
\begin{aligned}
\hat{g}_{\rho}\left(a, \tau_{2}\right) \equiv & -\frac{\mathcal{M}^{4}}{2} \int_{-1 / 2}^{1 / 2} d \tau_{1} \\
& \times\left\{\left[\sum_{m, n}(-1)^{F} \bar{q}^{m} q^{n}\right] \hat{\mathcal{G}}_{\rho}(a, \tau)\right\} \\
= & -\frac{\mathcal{M}^{4}}{2}\left[\operatorname{Str} e^{-\pi \alpha^{\prime} M^{2} \tau_{2}}\right] \hat{\mathcal{G}}_{\rho}\left(a, \tau_{2}\right) .
\end{aligned}
$$

On the second line the sum over $(m, n)$ indicates a sum over the entire spectrum of the theory, while in passing to the factorized form on the third line of Eq. (4.51) we have followed again the result in Eq. (3.37) and explicitly restricted our attention to those cases with $a \ll 1$, as appropriate for the regulator function $\hat{\mathcal{G}}_{\rho}(a, \tau)$. Indeed, the term within square brackets on the second line of Eq. (4.29) is our desired supertrace over physical string states, while the regulator function $\hat{\mathcal{G}}_{\rho}\left(a, \tau_{2}\right)$ provides a nontrivial $\tau_{2}$-dependent weighting to the different terms within $g_{\rho}\left(a, \tau_{2}\right)$.

Once again, the next step is to substitute Eq. (4.51) back into Eq. (4.50) and evaluate the residue at $s=1$. In general, the presence of the regulator function $\hat{\mathcal{G}}_{\rho}\left(a, \tau_{2}\right)$ within Eq. (4.29) renders this calculation somewhat intricate. However, just as for the terms with nontrivial $\mathcal{X}_{i}$ insertions, we know that $\hat{\mathcal{G}}_{\rho}\left(a, \tau_{2}\right) \rightarrow 1$ as $a \rightarrow 0$. In this limit, we therefore expect to obtain our original (finite) unregulated $\Lambda$ :

$$
\lim _{a \rightarrow 0} \hat{\Lambda}(\rho, a)=\Lambda=\frac{1}{24} \mathcal{M}^{2} \operatorname{Str} M^{2}
$$

where in the final equality we have utilized the result in Eq. (4.45). Equivalently, upon identifying the physical scale $\mu$ as in Eq. (4.21), we thus expect

$$
\lim _{\mu \rightarrow 0} \hat{\Lambda}(\mu)=\Lambda .
$$

Let us now determine how $\hat{\Lambda}(\mu)$ runs as a function of the scale $\mu$. To do this, we need to evaluate $\hat{\Lambda}(\rho, a)$ explicitly as a function of $\rho$ and $a$. This question is tackled in Appendix B, yielding the exact result in Eq. (B8). Written in terms of the physical scale $\mu$ in Eq. (4.33) this result then takes the form

$$
\begin{aligned}
\hat{\Lambda}(\mu)= & \frac{1}{1+\mu^{2} / M_{s}^{2}}\left\{\frac{\mathcal{M}^{2}}{24} \operatorname{Str} M^{2}-\frac{7}{960 \pi^{2}}\left(n_{B}-n_{F}\right) \mu^{4}\right. \\
& -\frac{1}{2 \pi^{2}} \operatorname{Str}_{M>0} M^{4}\left[\mathcal{K}_{1}^{(-1,0)}\left(\frac{2 \sqrt{2} \pi M}{\mu}\right)\right. \\
& \left.\left.+4 \mathcal{K}_{2}^{(-2,-1)}\left(\frac{2 \sqrt{2} \pi M}{\mu}\right)+\mathcal{K}_{3}^{(-1,0)}\left(\frac{2 \sqrt{2} \pi M}{\mu}\right)\right]\right\}
\end{aligned}
$$


where we have again taken $\rho=2$ as our benchmark value, where $\mathcal{K}_{\nu}^{(n, p)}(z)$ are the Bessel-function combinations defined in Eq. (4.36), and where $n_{B}$ and $n_{F}$ are the numbers of massless bosonic and fermionic degrees of freedom in the theory respectively (so that $\underset{M=0}{\operatorname{Str} \mathbf{1}}=n_{B}-n_{F}$ ).

It is straightforward to verify that this result is consistent with the result in Eq. (4.53) in the $\mu \rightarrow 0$ limit. Because all of the Bessel-function combinations within Eq. (4.54) vanish exponentially rapidly as their arguments grow to infinity, only the first term in Eq. (4.54) survives in this limit. We therefore find that the $\mu \rightarrow 0$ limit of Eq. (4.54) yields the anticipated result in Eq. (4.53).

From Eq. (4.54) we can also understand the manner in which $\hat{\Lambda}(\mu)$ runs as a function of $\mu$ for all $0<\mu \ll M_{s}$. Let us first focus on the Bessel-function terms within the square brackets in Eq. (4.54). By themselves, these terms behave in much the same way as shown in Fig. 2, except without the transient dip and with the asymptotic behavior for $\mu \gtrsim M$ scaling as a power (rather than logarithm) of $\mu$. More specifically, to leading order in $\mu / M$ and for $\mu \gtrsim M$, we find using the techniques developed in Ref. [28] that

$\mathcal{K}_{1}^{(-1,0)}(z)+4 \mathcal{K}_{2}^{(-2,-1)}(z)+\mathcal{K}_{3}^{(-1,0)}(z) \sim \frac{7}{480}\left(\frac{\mu}{M}\right)^{4}$

where $z \equiv 2 \sqrt{2} \pi M / \mu$. By contrast, for $\mu \lesssim M$, this quantity is exponentially suppressed. Thus, recalling the result in Eq. (4.45) for our original unregulated (but nevertheless finite) cosmological constant $\Lambda$ and once again keeping only those (leading) running terms which dominate for $M \ll \mu \ll M_{s}$, we find that Eq. (4.54) simplifies to take the approximate form

$$
\begin{aligned}
\hat{\Lambda}(\mu) & \approx \Lambda-\frac{7}{960 \pi^{2}}[(\underset{M=0}{\operatorname{Str} \mathbf{1}})+(\underset{0<M \lesssim \mu}{\operatorname{Str} \mathbf{1}})] \mu^{4} \\
& \approx \Lambda-\frac{7}{960 \pi^{2}}(\underset{0 \leq M \lesssim \mu}{\operatorname{Str}} \mathbf{1}) \mu^{4} .
\end{aligned}
$$

We once again emphasize that we have retained the second term (scaling as $\mu^{4}$ ) as this is the leading $\mu$-dependent term when $M \ll \mu \ll M_{s}$. Just as for $\left.\hat{m}_{\phi}^{2}(\mu)\right|_{\mathcal{X}}$, there also generally exist additional running terms which scale as $\mu^{2}$ and $\log \mu$, but these terms are subleading relative to the above $\mu^{4}$ term when $M \ll \mu \ll M_{s}$. We shall discuss these subleading terms further in Sec. V. Moreover, just as we saw for $\left.\hat{m}_{\phi}^{2}(\mu)\right|_{\mathcal{X}}$, the $\mu^{4}$ scaling behavior can be enhanced to an even greater effective power $\mu^{n}$ with $n>4$ if the spectrum of light states is sufficiently dense when taking the supertrace over string states. However, even this leading $\mu^{n}$ scaling is generally subleading compared with the constant term $\Lambda$. Thus the regulated quantity $\hat{\Lambda}(\mu)$-unlike $\left.\hat{m}_{\phi}^{2}(\mu)\right|_{\mathcal{X}}$ in Eq. (4.42) - is dominated by a constant term and exhibits at most a highly suppressed running relative to this constant.

\section{The Higgs mass in string theory: See how it runs}

We now finally combine both contributions $\left.\hat{m}_{\phi}^{2}(\mu)\right|_{\mathcal{X}, \Lambda}$ as in Eq. (4.25) in order to obtain our final result for the total modular-invariant regulated Higgs mass $\hat{m}_{\phi}^{2}(\mu)$. The exact result, of course, is given by the sum of Eqs. (4.35) and (4.54), with the latter first multiplied by $\xi /\left(4 \pi^{2} \mathcal{M}^{2}\right)$. However, once again taking the corresponding approximate forms in Eqs. (4.42) and (4.56) which are valid for $M \ll \mu \ll M_{s}$, we see that the $\mu^{4}$ running within Eq. (4.56) is no longer the dominant running for $\hat{m}_{\phi}^{2}(\mu)$ as a whole, as it is extremely suppressed compared with the running coming from Eq. (4.42). We thus find that to leading order, the net effect of adding Eqs. (4.42) and (4.56) is simply to add the overall constant $\xi \Lambda /\left(4 \pi^{2} \mathcal{M}^{2}\right)$ to the result in Eq. (4.42). We therefore find that the total regulated Higgs mass has the leading running behavior

$$
\begin{aligned}
\hat{m}_{\phi}^{2}(\mu) \approx & \frac{\xi}{4 \pi^{2}} \frac{\Lambda}{\mathcal{M}^{2}}-\frac{\pi}{6} \mathcal{M}^{2} \operatorname{Str} \mathbb{X}_{1} \\
& +\mathcal{M}^{2} \underset{M=0}{\operatorname{Str}} \mathbb{X}_{2} \log \left(\frac{\mu}{2 \sqrt{2} e M_{s}}\right) \\
& +\mathcal{M}^{2} \underset{0<M \lesssim \mu}{\operatorname{Str}} \mathbb{X}_{2} \log \left[\frac{1}{\sqrt{2}} e^{-(\gamma+1)} \frac{\mu}{M}\right]
\end{aligned}
$$

where we have retained only the terms that are leading for $M \ll \mu \ll M_{s}$. Once again, just as for $\left.\hat{m}_{\phi}^{2}(\mu)\right|_{\mathcal{X}}$, we see that to this order the $\mathbb{X}_{2}$ charges of the string states lead to nontrivial running while their $\mathbb{X}_{1}$ charges only contribute to an overall additive constant. Indeed, in the $\mu \rightarrow 0$ limit, we find

$$
\lim _{\mu \rightarrow 0} \hat{m}_{\phi}^{2}(\mu)=\frac{\xi}{4 \pi^{2}} \frac{\Lambda}{\mathcal{M}^{2}}-\frac{\pi}{6} \mathcal{M}^{2} \operatorname{Str} \mathbb{X}_{1}
$$

when $\underset{M=0}{\operatorname{Str}} \mathbb{X}_{2}=0$. Of course, when $\underset{M=0}{\operatorname{Str}} \mathbb{X}_{2} \neq 0$, the $\mu \rightarrow 0$ limit diverges, as expected from the fact that the massless $\mathbb{X}_{2}$-charged states are precisely the states that led to a divergence in the original unregulated Higgs mass $m_{\phi}^{2}$. As discussed in Sec. IV E 1, we nevertheless continue to obtain a finite result for the regulated Higgs mass $\hat{m}_{\phi}^{2}(\mu)$ for all $\mu>0$ even when $\underset{M=0}{\operatorname{Str}} \mathbb{X}_{2} \neq 0$.

In order to understand what the running in Eq. (4.57) looks like for $0<\mu \ll M_{s}$, let us begin by considering the contribution from a single $\mathbb{X}_{2}$-charged string state with a given mass $0<M \ll M_{s}$. In this case, we have $\underset{M=0}{\operatorname{Str}} \mathbb{X}_{2}=0$. It then follows that the approximate form in Eq. (4.57) reduces to 


$$
\begin{aligned}
\left.\hat{m}_{\phi}^{2}(\mu)\right|_{\mathcal{X}} \approx & \frac{\xi}{4 \pi^{2}} \frac{\Lambda}{\mathcal{M}^{2}}-\frac{\pi}{6} \mathcal{M}^{2} \operatorname{Str} \mathbb{X}_{1} \\
& +\mathcal{M}^{2} \underset{0<M \lesssim \mu}{\operatorname{Str}} \mathbb{X}_{2} \log \left[\frac{1}{\sqrt{2}} e^{-(\gamma+1)} \frac{\mu}{M}\right]
\end{aligned}
$$

where $\Lambda$ now represents the contribution to the total cosmological constant from this single state and where the supertraces $\operatorname{Str} \mathbb{X}_{1,2}$ now simply reduce to the (statisticsweighted) $\mathbb{X}_{1,2}$ charges of that state. For $\mu \gg M$, the final term in Eq. (4.59) produces a logarithmic running. Of course, the approximate result in Eq. (4.59) is valid only for $\mu \gg M$. For $\mu \ll M$, we instead know that our running asymptotically approaches the constant in Eq. (4.58). Likewise, for $\mu \sim M$, we know that the running interpolates between these two behaviors via the transient "dip" shown in Fig. 2.

With all of these pieces combined, the net running contributed from a single bosonic $\mathbb{X}_{2}$-charged state of mass $M$ has the behavior sketched as the green curve within the $\mu \ll M_{s}$ portion of Fig. 3, where we interpret $M_{\text {lightest }} \sim M$. Indeed, this curve is essentially the same as that shown in Fig. 2, but with the addition of the asymptotic constant in Eq. (4.58).

Given this result from a single state of mass $M$, we now must take the supertrace over the entire spectrum of states in the theory. However, as discussed in Sec. IV E 1, increasing (decreasing) the mass of the contributing state simply shifts the corresponding contribution rigidly to the right (left). Taking the supertrace then simply amounts to adding these different shifted contributions together, weighted by their corresponding $\mathbb{X}_{2}$ charges and statistics factors. Of course, as discussed in Sec. IV E 1, heavy states whose masses exceed $\mu$ are effectively integrated out of the theory: they contribute to the overall asymptotic constant in Eq. (4.58) but produce no effective running beyond this. For any value of $\mu$ we therefore need only sum over the contributions from those light states whose masses lie below $\mu$.

The net result of this summation over string states is as follows. As explained in the discussion surrounding Eq. (4.43), this summation has the potential to turn the logarithmic running into a power-law running for scales $\mu$ which lie within the spectrum of masses of the light $\mathbb{X}_{2}$-charged states-i.e., for scales $\mu>M_{\text {lightest }}$, where $M_{\text {lightest }}$ denotes the mass of the lightest $\mathbb{X}_{2}$-charged states. Indeed, as discussed previously, whether an effective power-law running emerges depends on the density of states in the theory with masses $M \gtrsim M_{\text {lightest }}$. It is for this reason that we have indicated in Fig. 3 that the net running within the $\mu>M_{\text {lightest }}$ region can be either logarithmic or power-law. However, as we progress to lower scales $\mu \sim M_{\text {lightest }}$, we enter the "dip region" where this logarithmic/power-law running shuts off. Finally, for $\mu<M_{\text {lightest }}$, all running ceases as we enter the deep infrared region. It is here that we recover the asymptotic constant value predicted in Eq. (4.58), which now represents the sum over the individual asymptotic contributions from all of the string states. Note that this implies that the unregulated integral represents the IR value of the Higgs mass.

Depending on the relative values of $\Lambda, \operatorname{Str} \mathbb{X}_{1}$, and $\underset{M>0}{\operatorname{Str}} \mathbb{X}_{2}$, the Higgs may actually become tachyonic within $M>0$

the "dip" region. Possible phenomenological implications of this will be briefly discussed in Sec. VI. Of course, if $M_{\text {lightest }}$ is exceedingly small (or similarly if $\underset{M=0}{\operatorname{Str}} \mathbb{X}_{2} \neq 0$ ), we never hit the dip region or the asymptotic-constant region. In this case, our EFT-like logarithmic/power-law running persists all the way into the deep infrared (as indicated through the dashed blue line in Fig. 3).

All of these results are valid for the same "infrared" region in which our regulator itself is valid, namely the region with $a \ll 1$ or equivalently $\mu \ll M_{s}$. However, it turns out that we also have information about what happens in the opposite region, namely that with $\mu \gg M_{s}$ : as sketched in Fig. 3, we simply enter a "dual infrared" region in which this same infrared behavior again emerges, but in reverse. This is a direct consequence of the modular invariance which we have been careful to maintain throughout our calculations. Indeed, modular invariance ensures that this entire picture is symmetric under the scale-inversion duality transformation

$$
\mu \rightarrow \frac{M_{s}^{2}}{\mu}
$$

As a result, when plotted as a function of $\log \left(\mu / M_{s}\right)$, the behavior of $\hat{m}_{\phi}^{2}(\mu)$ for $\mu \ll M_{s}$ is reflected symmetrically through the self-dual point $\mu_{*}=M_{s}$ to yield the reverse behavior as $\mu \gg M_{s}$. Of course, this tells us nothing about the behavior of $\hat{m}_{\phi}^{2}(\mu)$ near the self-dual region with $\mu \sim M_{s}$, except that any running at the self-dual point $\mu_{*}=$ $M_{s}$ must ultimately become exactly flat. We have sketched one possible shape for this running with a dashed (rather than solid) curve within the self-dual region in Fig. 3.

The origins of the scale-duality symmetry in Eq. (4.60) are easily understood. We have seen in Eq. (3.40) that our regulator functions $\hat{G}_{\rho}(a, \tau)$ - and hence our regulated Higgs masses $\hat{m}_{\phi}^{2}(\rho, a)$ - have an invariance under $\rho a^{2} \rightarrow$ $1 /\left(\rho a^{2}\right)$. Likewise, we have seen in Eq. (4.33) that the running scale $\mu^{2}$ is given by $\rho a^{2} M_{s}^{2}$. These two relations then directly imply that $\hat{m}_{\phi}^{2}(\mu)$ is invariant under the scaleduality transformation in Eq. (4.60). However, the origins of this scale-duality symmetry actually run deeper than any particular modular-invariant regulator we might choose, and are directly connected to the underlying modular invariance of theory. To see this, we recall from the discussion surrounding Eqs. (4.19) and (4.20) that the 


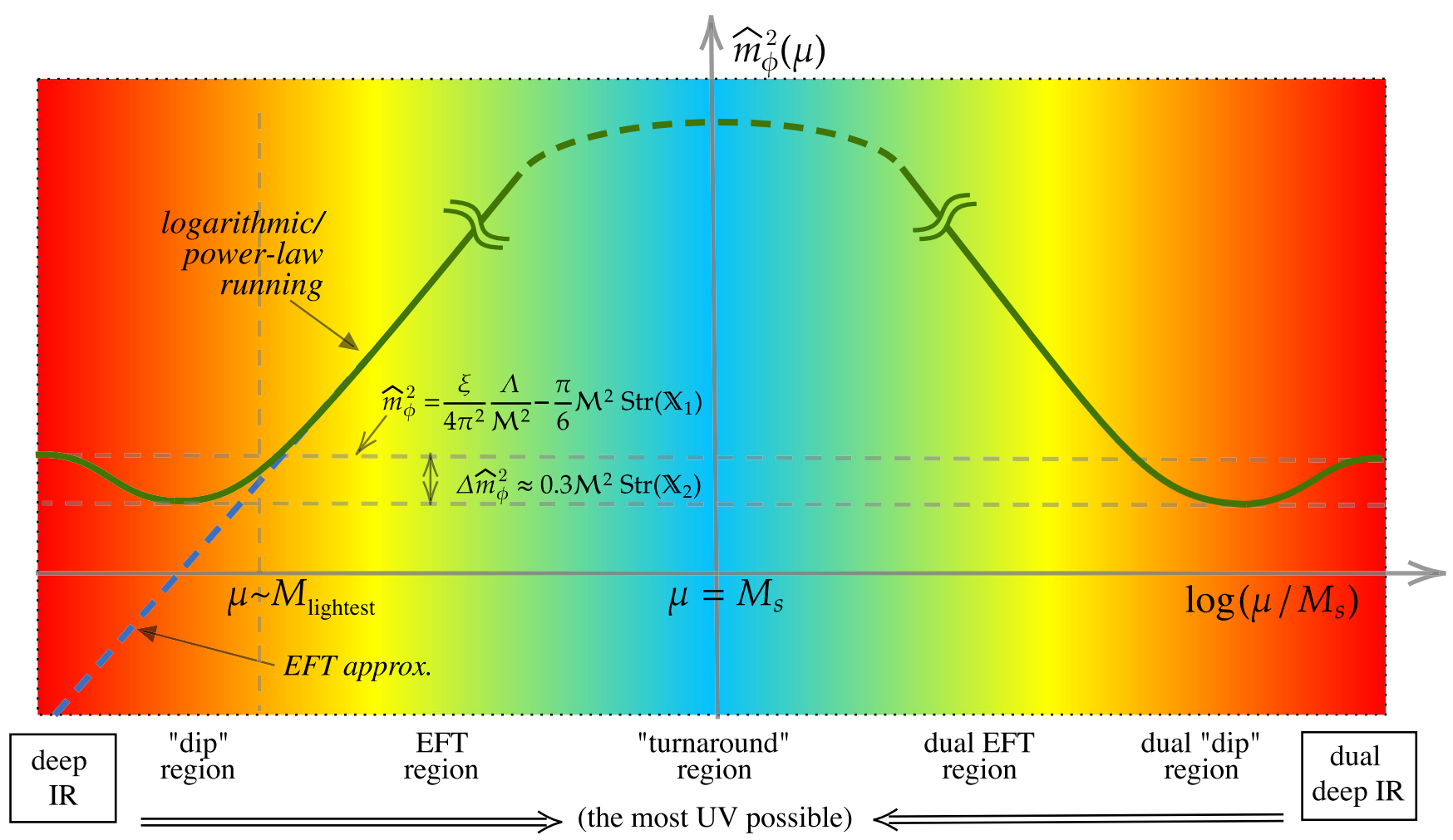

FIG. 3. The "running" of the regulated Higgs mass $\hat{m}_{\phi}^{2}(\mu)$, as calculated from first principles in a fully modular-invariant string framework. As discussed in the text, this running (green curve) exhibits a rather complicated anatomy. In the deep infrared (as $\mu \rightarrow 0$ ), the Higgs mass approaches an asymptotic value which depends on the cosmological constant of the theory as well as on the supertrace over the $\mathbb{X}_{1}$ charges of all of the string states. Moving toward higher values of $\mu$, a nontrivial scale-dependence does not emerge until $\mu \sim M_{\text {lightest }}$, where $M_{\text {lightest }}$ collectively represents the masses of the lightest massive $\mathbb{X}_{2}$-charged states. The "dip" that is observed in this region is a string-theoretic transient effect which smoothly connects the asymptotic deep-IR region $\left(\mu \ll M_{\text {lightest }}\right)$ to an EFT-like region $\left(M_{\text {lightest }} \lesssim \mu \ll M_{s}\right)$. Moving beyond this dip region, the theory then enters the EFT-like region in which the Higgs mass experiences a running which is either logarithmic or power-law, depending on the density of string states with masses in the range $M_{\text {lightest }} \lesssim M \ll M_{s}$. Note that this logarithmic/power-law running behavior will actually persist all the way into the deep infrared (dashed blue curve) if $M_{\text {lightest }}$ is exceedingly small. Finally, as $\mu$ approaches $M_{s}$, the regulator we have employed is no longer valid and thus we cannot explicitly calculate the running. One possible running is sketched (dashed green curve). However, as a general principle, modular invariance requires that the running of the Higgs mass (and indeed the running of any physical quantity) exhibit an invariance under $\mu \rightarrow M_{s}^{2} / \mu$. Thus, as $\mu$ increases beyond $M_{s}$, we inevitably begin to reenter an IR-like regime which we may associate with a "dual" EFT. The background colors of this sketch indicate the transition from the deep IR (red) to the UV (blue) and then back to IR (red). This symmetry under $\mu \rightarrow M_{s}^{2} / \mu$ implies that the self-dual scale $\mu \sim M_{s}$ exhibits the "maximum possible" UV behavior, in the sense that further increases in $\mu$ only serve to push the theory back toward IR behavior. Of course, as discussed in Sec. IVA, a fully modular-invariant string theory does not distinguish between the UV and the IR. Indeed, the UV and IR labels in this figure only arise upon attempting to extract a field-theoretic description of our theory, as we are implicitly doing when discussing the "running" of the Higgs mass.

contributions of string states of mass $M$ to the one-loop partition function experience Boltzmann suppressions scaling as $e^{-\pi \alpha^{\prime} M^{2} \tau_{2}}$. Thus, for any particular benchmark value $\tau_{2}=t$, we can separate our string spectrum into two groups: "heavy" states (whose Boltzmann suppressions at $\tau_{2}=t$ are significant according to some convention, and whose contributions therefore do not require regularization) and "light" states (whose Boltzmann suppressions are not significant, and whose contributions therefore require regularization). Indeed, taking $t \rightarrow \infty$ ensures that the only states whose contributions to the partition function remain unsuppressed are those which are strictly massless. On this basis, with an eye toward extracting an EFT with a running scale $\mu$, we are directly led to identify $\mu^{2}$ inversely with $t$, as in Eq. (4.20). However, modular invariance tells us that any physical quantities which depend on $\tau$ must be invariant under $\tau \rightarrow-1 / \tau$. Along the $\tau_{1}=0$ axis, this becomes an invariance under $\tau_{2} \rightarrow 1 / \tau_{2}$. This then immediately implies an invariance under $t \rightarrow 1 / t$, or equivalently under $\mu \rightarrow \mu_{*}^{2} / \mu$ where $\mu_{*}$ is an arbitrary self-dual mass scale. Of course, the choice of normalization for $\mu$ in relation to $t$ is purely a matter of convention, and for 
convenience in this paper we have chosen our normalization for $\mu$ such that $\mu_{*}=M_{s}$. We thus see that while the particular choice of self-dual scale $\mu^{*}$ is a matter of convention, the existence of a scale-inversion duality symmetry of the form $\mu \rightarrow \mu_{*}^{2} / \mu$ is inevitable, emerging directly from the underlying modular invariance of the theory. This issue will be discussed further in Sec. VI.

Although this scale-duality symmetry follows directly from modular invariance, its implications are profound. Ultimately, the existence of such a symmetry signals the existence of an ultimate limit on the extent to which our EFT way of thinking can possibly remain valid in string theory. Indeed, as discussed in the Introduction, string theory is rife with duality symmetries which defy EFT notions: an immediate example of this is T-duality, under which the physics associated with a closed string propagating on a spacetime with a compactified dimension of radius $R$ is indistinguishable from the physics associated with a closed string propagating on a spacetime with a compactified dimension of radius $R^{\prime} \equiv \alpha^{\prime} / R$. This is true as an exact symmetry not only for the string spectrum but also for all interactions. Thus such strings cannot distinguish between large and small compactification geometries, thereby preventing us from establishing a linear EFT-like ordering of length scales from large to small, or equivalently from IR to UV. What we are seeing now is that a similar phenomenon is guaranteed by modular invariance. Although we can identify $\mu \ll M_{s}$ as the deep-infrared region of our EFT, and although we may legitimately identify the passage toward larger scales $\mu$ as a passage toward an increasingly UV region of this EFT, we see that the validity of this identification has a fundamental limit. Indeed, pushing $\mu$ beyond $M_{s}$ only serves to reintroduce the original IR behavior of our theory-a behavior which we may now associate with the dual energy scale $\mu^{\prime} \equiv M_{s}^{2} / \mu$ associated with a "dual" EFT. In this sense, the energy scales near $M_{s}$ exhibit the "most possible UV" behavior that can be realized. This is indicated through the background colors of Fig. 3, with red indicating the IR regions of our theory and blue indicating the UV.

As we have emphasized, all of this is the inevitable consequence of modular invariance. At first glance, it may not seem that our results for $\hat{m}_{\phi}^{2}(\mu)$ are modular invariant, much less symmetric under the scale-duality symmetry in Eq. (4.60). Indeed, this symmetry is hardly manifest within our exact expressions for $\left.\hat{m}_{\phi}^{2}(\mu)\right|_{\mathcal{X}}$ and $\hat{\Lambda}(\mu)$ in Eqs. (4.35) and (4.54), respectively. However, this symmetry is ultimately ensured through intricate relations satisfied by the various supertraces involved. Indeed, such relations are themselves manifestations of the underlying modular invariance of the theory. In this paper we have not focused on the identities satisfied by these supertraces. However, we have already seen two such identities, namely the simple expressions for Str 1 and $\operatorname{Str} M^{2}$ in Eqs. (4.47) and (4.45), respectively. These identities, which were originally derived in Ref. [18], hold in any modular-invariant string theory, arising as a consequence of the so-called "misaligned supersymmetry" [17] that tightly constrains the distributions of bosonic and fermionic states across any modular-invariant string spectrum. In a similar vein, there will also exist identities satisfied by all of the supertraces that appear within Eqs. (4.35) and (4.54), especially when $\mathbb{X}_{i}$ insertions are involved. These identities are likely to be more intricate and interwoven than those for $\operatorname{Str} 1$ and $\operatorname{Str} M^{2}$, but together they act to ensure the modular invariance of our results for $\hat{m}_{\phi}^{2}(\mu)$.

We conclude, then, that the duality symmetry in Eq. (4.60) is a fundamental property of the running of any physical quantity in a modular-invariant theory. As such, there is a maximum degree to which our theory can approach the UV: once the energy scale $\mu$ passes the selfdual point $\mu \sim M_{s}$, further increases in $\mu$ only push us toward increasingly IR behavior. Of course, as we explained in Sec. IVA, the full modular-invariant string theory does not distinguish between the UV and IR-this distinction only has meaning when we attempt to extract an EFT description, as we are doing when discussing the "running" of the Higgs mass. Such, then, are the unique properties of UV/IR-mixed theories such as those exhibiting modular invariance.

\section{TRANSCENDING THE CHARGE LATTICE}

Thus far in this paper, our guide has been modular invariance-an exact symmetry of closed strings. However, right from the beginning of Sec. II, we have further assumed that our closed-string models have associated charge lattices. This has been the language of our analysis, and as such this has given our calculations a certain concreteness, enabling us to obtain and express our main results in a rather direct and understandable fashion as the supertrace of physical string states weighted by their eigenvalues with respect to certain combinations of world sheet charge operators. This language also allowed us to understand the origins of the modular anomaly that ultimately connected the Higgs mass and the cosmological constant.

It is certainly the case that many classes of closed-string models can be described in terms of their associated charge lattices. For example, charge lattices appear for a wide variety of geometric compactifications and thus play an essential role in many corresponding free-field constructions (such as those based on free world sheet bosons and complex fermions). However, not all string theories can be described in this manner.

Fortunately, in hindsight, it is not difficult to demonstrate that our results are actually general and do not ultimately rely on the existence of such charge lattices. Indeed, as we shall now demonstrate, most of our results follow from modular invariance alone, and can be expressed in a more 
general language that makes no specific reference to charge operators. Thus, phrasing our results in this more general language demonstrates that our results actually transcend their charge-lattice roots and have a more encompassing generality. This will also help us discern the existence of a "stringy" effective potential for the Higgs.

\section{A. X's without Q's: A reformulation of the partition-function insertions}

In Sec. II, we established a framework for calculating the Higgs mass in which the charge lattice played a central role. By extracting the $\eta$ functions as explicit prefactors in Eq. (2.1), we implicitly separated out the contributions from the oscillator modes and thereby implicitly cast the partition function of our theory into a form in which the $(m, n)$ exponents in Eq. (2.1) were directly related to the lengths of the charge vectors in an underlying charge lattice, as in Eq. (2.2). By contrast, the spacetime masses $\left(M_{L}, M_{R}\right)$ of the corresponding string states receive contributions from not only the charges (i.e., the compactification momentum modes) but also the oscillator modes within the $\eta$ 's. Indeed, these contributions are ultimately added together, as in Eq. (2.5).

Taken together, this means that we can always rewrite our partition function $\mathcal{Z}$ in a general form which is closer to what appears in Eq. (3.2), namely

$$
\mathcal{Z}(\tau)=\tau_{2}^{-1} \sum_{\text {states }}(-1)^{F} \bar{q}^{\alpha^{\prime} M_{R}^{2} / 4} q^{\alpha^{\prime} M_{L}^{2} / 4}
$$

where we are summing over the states in the string spectrum with left- and right-moving spacetime mass contributions $\left(M_{L}, M_{R}\right)$. Although Eq. (5.1) reduces to Eq. (2.1) for theories with a charge lattice, the expression in Eq. (5.1) is more general and applies to any closed string. Just as in Sec. II, this partition function is assumed to describe the theory in its Higgsed phase.

Following the arguments in Sec. II, we seek to evaluate the Higgs mass by exploring the response of the theory to small fluctuations $\phi$ of the Higgs field around its minimum $\langle\phi\rangle$. Previously we described the response of the system in terms of a deformation of the charge lattice, as in Eq. (2.13). However, we may more generally simply describe the response of our theory in terms of the corresponding deformations to our left- and right-moving masses, so that $M_{L}$ and $M_{R}$ for a given string state $s$ now become functions of $\phi$ :

$$
\begin{aligned}
& M_{L}^{(s)} \rightarrow M_{L}^{(s)}+\delta M_{L}^{(s)}(\phi) \equiv M_{L}^{(s)}(\phi), \\
& M_{R}^{(s)} \rightarrow M_{R}^{(s)}+\delta M_{R}^{(s)}(\phi) \equiv M_{R}^{(s)}(\phi) .
\end{aligned}
$$

Of course, for a given Higgs field, not all states in the string spectrum will have their masses shifted. Indeed, mass shifts will arise for only those states which couple to the fluctuations parametrized by $\phi$; the masses of the other states will remain independent of $\phi$. It is for this reason that we have explicitly attached a state index $s$ to the masses in Eq. (5.2) —namely to clarify that the mass shifts depend on the particular state $s$ and not merely on the unperturbed masses $\left(M_{L}, M_{R}\right)$. This is completely analogous to the fact that only certain charge vectors in Eq. (2.13) will be deformed. In the following, for the sake of parallelism with Eq. (2.13), we shall suppress the state index $s$ in Eq. (5.2) with the understanding that whether certain $M_{L, R}(\phi)$ are truly $\phi$-dependent depends not on $M_{L, R}$ but rather on the identity of the state $s$ from which these contributions emerge. In this context, we remark that changing the value of $\phi$ generally does more than shift the masses of certain states-it will also typically mix these states, thereby changing the corresponding mass eigenstates. We should thus understand the index $s$ as continuously following a given mass eigenstate as $\phi$ is changed.

Just as in the charge-vector formalism, the choices of $\delta M_{L, R}(\phi)$ are not arbitrary; modular invariance [in this case, the invariance of $\mathcal{Z}(\phi)$ under $\tau \rightarrow \tau+1$ ] must still be maintained. This implies that

$$
\delta M_{L}(\phi)=\delta M_{R}(\phi),
$$

which is the generalization of the constraints in Eq. (2.14).

Given these observations, we can then calculate the Higgs mass precisely as in Eqs. (2.22) and (2.23), except with $\mathcal{Z}$ expressed as in Eq. (5.1) with the masses $M_{L}$ and $M_{R}$ replaced by their $\phi$-dependent versions $M_{L}(\phi)$ and $M_{R}(\phi)$. We then have

$$
\frac{\partial^{2} \mathcal{Z}}{\partial \phi^{2}}=\tau_{2}^{-1} \sum_{\text {states }}(-1)^{F} X \bar{q}^{\alpha^{\prime} M_{R}^{2} / 4} q^{\alpha^{\prime} M_{L}^{2} / 4}
$$

where the summand insertion $X$ is precisely the same quantity as in Eq. (2.27) but now expressed as

$X \equiv \frac{\pi i \alpha^{\prime}}{2} \partial_{\phi}^{2}\left(\tau M_{L}^{2}-\bar{\tau} M_{R}^{2}\right)-\left(\frac{\pi \alpha^{\prime}}{2}\right)^{2}\left[\partial_{\phi}\left(\tau M_{L}^{2}-\bar{\tau} M_{R}^{2}\right)\right]^{2}$.

However, each term within Eq. (5.5) with a nonzero power of $\tau_{1}$ also contains equally many powers of $M_{L}^{2}-M_{R}^{2}$, and we see from Eqs. (5.2) and (5.3) that

$$
\partial_{\phi}\left(M_{L}^{2}-M_{R}^{2}\right)=\partial_{\phi}\left(\delta M_{L}^{2}-\delta M_{R}^{2}\right)=0 .
$$

Thus each factor of $\tau M_{L}^{2}-\bar{\tau} M_{R}^{2}$ within Eq. (5.5) can be replaced with $i \tau_{2}\left(M_{L}^{2}+M_{R}^{2}\right)=2 i \tau_{2} M^{2}$ where $M$ is the ( $\phi$-dependent) shifted spacetime mass of the corresponding state. This then yields 


$$
X=-\pi \alpha^{\prime} \tau_{2} \partial_{\phi}^{2} M^{2}+\left(\pi \alpha^{\prime} \tau_{2}\right)^{2}\left(\partial_{\phi} M^{2}\right)^{2} .
$$

Recalling $\left.\mathcal{X} \equiv \mathcal{M}^{2} X\right|_{\phi=0}$ and separating out the different powers of $\tau_{2}$ in Eq. (5.7) then immediately enables us to identify

$$
\begin{aligned}
& \mathbb{X}_{1}=-\left.\frac{1}{4 \pi} \partial_{\phi}^{2} M^{2}\right|_{\phi=0}, \\
& \mathbb{\searrow}_{2}=\left.\frac{1}{16 \pi^{2} \mathcal{M}^{2}}\left(\partial_{\phi} M^{2}\right)^{2}\right|_{\phi=0} .
\end{aligned}
$$

These, then, are the general forms of the $\mathbb{X}_{i}$ insertionsforms which do not rely on the existence of a charge lattice. As indicated above, only for those string states which couple to the Higgs will the corresponding spacetime masses $M$ be affected by fluctuations of the Higgs field and thereby accrue a $\phi$-dependence. Thus, given the $\phi$-derivatives in Eq. (5.8), we see that only these states will contribute to $\mathbb{X}_{1}$ and $\mathbb{X}_{2}$. Of course, when the string model in question has an underlying charge lattice, the masses of our string states can be written in terms of the lengths of the vectors in that lattice, whereupon the results in Eq. (5.8) can be evaluated to have the more specific forms presented in earlier sections.

Given the general results in Eq. (5.8), the rest of our analysis proceeds precisely as before. The $\phi$-derivatives generally induce modular anomalies that require modular completions and lead us to add the universal $\Lambda$-term, as before. Our final result for the unregulated Higgs mass from Eq. (2.55) then takes the form

$$
\begin{aligned}
m_{\phi}^{2}= & \frac{\xi}{4 \pi^{2}} \frac{\Lambda}{\mathcal{M}^{2}}-\frac{\mathcal{M}^{2}}{2}\left\langle\tau_{2} \mathbb{X}_{1}+\tau_{2}^{2} \mathbb{X}_{2}\right\rangle \\
= & \frac{\xi}{4 \pi^{2}} \frac{\Lambda}{\mathcal{M}^{2}}+\frac{\mathcal{M}^{2}}{8 \pi}\left\langle\left.\tau_{2} \partial_{\phi}^{2} M^{2}\right|_{\phi=0}\right\rangle \\
& -\frac{1}{32 \pi^{2}}\left\langle\left.\tau_{2}^{2}\left(\partial_{\phi} M^{2}\right)^{2}\right|_{\phi=0}\right\rangle
\end{aligned}
$$

where the bracket notation $\langle\ldots\rangle$ is defined in Eq. (2.52). This result can then be regulated, as discussed in Sec. IV, leading to a regulated Higgs mass $\hat{m}_{\phi}^{2}(\rho, a)$. Indeed, none of the results presented after Sec. II depended on the specific forms of $\mathbb{X}_{1}$ and $\mathbb{X}_{2}$ in any way.

\section{B. A stringy effective potential for the Higgs}

As we shall now demonstrate, the results in Eq. (5.8) allow us to reach some powerful conclusions about the regulated Higgs mass $\hat{m}_{\phi}^{2}(\rho, a)$. They will also allow us to extract a stringy effective potential for the Higgs. We begin by noting that $\hat{m}_{\phi}^{2}(\rho, a)$ has two contributions, $\left.\hat{m}_{\phi}^{2}(\rho, a)\right|_{\mathcal{X}}$ and $\left.\hat{m}_{\phi}^{2}(\rho, a)\right|_{\Lambda}$, as indicated in Eq. (4.25). The first of these contributions involves $\mathbb{X}_{i}$ insertions, while the second comes from the cosmological constant and lacks such insertions. Of course, both contributions depend on the spectrum of masses of the states in our theory-masses which we are now taking to be general functions of $\phi$ before eventually truncating to $\phi=0$. However, we see from Eq. (5.8) that the $\mathbb{X}_{i}$ insertions depend on the derivatives of these masses with respect to $\phi$, whereas no such derivatives appear within $\left.\hat{m}_{\phi}^{2}(\rho, a)\right|_{\Lambda}$. Thus suggests that $\left.\hat{m}_{\phi}^{2}(\rho, a)\right|_{\mathcal{X}}$ might be related to $\phi$-derivatives of $\left.\hat{m}_{\phi}^{2}(\rho, a)\right|_{\Lambda}$ prior to the truncation to $\phi=0$. If so, the two Higgs-mass contributions $\left.\hat{m}_{\phi}^{2}(\rho, a)\right|_{\mathcal{X}}$ and $\left.\hat{m}_{\phi}^{2}(\rho, a)\right|_{\Lambda}$ would actually be deeply connected to each other.

To investigate this possibility, we note from Appendices A and B that these two contributions to the Higgs mass can be identically expressed in terms of simpler quantities, $P_{\mathcal{X}}$ and $P_{\Lambda}$, via the relations

$$
\begin{aligned}
\left.\hat{m}_{\phi}^{2}(\rho, a)\right|_{\mathcal{X}} & =\frac{1}{1+\rho a^{2}} A_{\rho} a^{2} \frac{\partial}{\partial a}\left[P_{\mathcal{X}}(\rho a)-P_{\mathcal{X}}(a)\right], \\
\hat{\Lambda}(\rho, a) & =\frac{1}{1+\rho a^{2}} A_{\rho} a^{2} \frac{\partial}{\partial a}\left[P_{\Lambda}(\rho a)-P_{\Lambda}(a)\right]
\end{aligned}
$$

where $A_{\rho} \equiv \rho /(\rho-1)$, where we recall $\left.\hat{m}_{\phi}^{2}(\rho, a)\right|_{\Lambda} \equiv$ $\xi \hat{\Lambda}(\rho, a) /\left(4 \pi^{2} \mathcal{M}^{2}\right)$, and where $P_{\mathcal{X}}(a)$ and $P_{\Lambda}(a)$ are given in Eqs. (A15) and (B7) respectively. Since $\phi$-derivatives commute with the operators in Eq. (5.10), the question of whether $\left.\hat{m}_{\phi}^{2}(\rho, a)\right|_{\mathcal{X}}$ might be related to $\phi$-derivatives of $\left.\hat{m}_{\phi}^{2}(\rho, a)\right|_{\Lambda}$ then boils down to whether $P_{\mathcal{X}}(a)$ might be related to $\phi$-derivatives of $P_{\Lambda}(a)$, where each mass $M$ is now to be regarded as a function of $\phi$ prior to truncation.

Our procedure, then, will be to evaluate $\phi$-derivatives of $P_{\Lambda}(a)$ in Eq. (B7) and determine the extent to which we reproduce $P_{\mathcal{X}}(a)$ in Eq. (A15). To do this, we shall now treat each squared mass $M^{2}$ within $P_{\Lambda}(a)$ in Eq. (B7) as representing the $\phi \rightarrow 0$ limit of a function $M^{2}(\phi)$ which, for $\phi / \mathcal{M} \ll 1$, we can imagine Taylor-expanding to take the generic form ${ }^{1}$

$M^{2}(\phi)=\mathcal{M}^{2}\left[\beta_{0}+\beta_{1}\left(\frac{\phi}{\mathcal{M}}\right)+\frac{1}{2} \beta_{2}\left(\frac{\phi}{\mathcal{M}}\right)^{2}+\ldots\right]$

where $\beta_{0} \geq 0$. The original physical squared mass of the state then corresponds to $\left.M^{2}(\phi)\right|_{\phi=0}=\beta_{0} \mathcal{M}^{2}$, so that massive states correspond to functions with $\beta_{0}>0$ while massless states have $\beta_{0}=0$. However, we see that even massless states introduce a $\phi$-dependence prior to the truncation to $\phi=0$.

\footnotetext{
${ }^{1}$ It is significant that we are Taylor-expanding $M^{2}$ rather than $M$. In closed-string theories, $M^{2}$ is the fundamental quantity, with world sheet excitations making contributions directly to $\alpha^{\prime} M^{2}$ as in Eq. (2.5). As we shall shortly see when discussing the stability of these theories, it is also significant that our Taylor expansion for $M^{2}$ generically includes a term linear in $\phi$. This follows directly from Eq. (2.13) in conjunction with Eqs. (2.4) and (2.5).
} 
Focusing initially on the first term within $P_{\Lambda}(a)$ in Eq. (B7), we immediately see that

$$
\begin{aligned}
\left.\partial_{\phi}^{2}\left[\frac{\mathcal{M}^{2}}{24 a} \operatorname{Str} M^{2}\right]\right|_{\phi=0} & =\left.\frac{\mathcal{M}^{2}}{24 a} \operatorname{Str}\left(\partial_{\phi}^{2} M^{2}\right)\right|_{\phi=0} \\
& =-\frac{\mathcal{M}^{2}}{2}\left[\frac{\pi}{3 a} \operatorname{Str} \mathbb{X}_{1}\right]
\end{aligned}
$$

where we have used the result in Eq. (5.8) in passing to the final expression. This successfully reproduces the initial terms within $P_{\mathcal{X}}(a)$ in Eq. (A15). Next, we evaluate the second $\phi$-derivative of the Bessel-function terms within $P_{\Lambda}(a)$ in Eq. (B7). To do this, we note the mathematical identity

$$
\begin{aligned}
\partial_{\phi}^{2}\left[M^{2} K_{2}\left(\frac{r M}{a \mathcal{M}}\right)\right]= & -\frac{r M}{2 a \mathcal{M}}\left(\partial_{\phi}^{2} M^{2}\right) K_{1}\left(\frac{r M}{a \mathcal{M}}\right) \\
& +\frac{r^{2}}{4 a^{2} \mathcal{M}^{2}}\left(\partial_{\phi} M^{2}\right)^{2} K_{0}\left(\frac{r M}{a \mathcal{M}}\right)
\end{aligned}
$$

which follows from standard results for Bessel-function derivatives along with a judicious repackaging of terms. Given this, and given the relations in Eq. (5.8), we then find that

$$
\begin{aligned}
\partial_{\phi}^{2}\{ & \left.\frac{a}{\pi^{2}} \operatorname{Str}_{\beta_{0}>0}\left[M^{2} \sum_{r=1}^{\infty} \frac{1}{r^{2}} K_{2}\left(\frac{r M}{a \mathcal{M}}\right)\right]\right\}\left.\right|_{\phi=0} \\
= & \frac{2}{\pi} \underset{M>0}{\operatorname{Str}} \mathbb{X}_{1}\left[\sum_{r=1}^{\infty}\left(\frac{M}{r \mathcal{M}}\right) K_{1}\left(\frac{r M}{a \mathcal{M}}\right)\right] \\
& +\frac{4}{a} \operatorname{Str}_{M>0} \mathbb{X}_{2}\left[\sum_{r=1}^{\infty} K_{0}\left(\frac{r M}{a \mathcal{M}}\right)\right],
\end{aligned}
$$

where the supertrace on the first line is over all states whose mass functions $M^{2}(\phi)$ have $\beta_{0}>0$. We thus see that the result in Eq. (5.14) likewise successfully reproduces the Bessel-function terms within $P_{\mathcal{X}}(a)$ in Eq. (A15).

Our final task is to evaluate $\partial_{\phi}^{2}$ acting on the second term in Eq. (B7). At first glance, it would appear that this term does not yield any contribution since it is wholly independent of the mass $M$ and would thus not lead to any $\phi$-dependence. Indeed, as evident from Eq. (B5), this term represents a contribution to $P_{\Lambda}(a)$ from purely massless states, and as such the identification $M=0$ has already been implemented within this term. This is why no factors of the mass $M$ remain within this term. However, as discussed above, for the purposes of the present calculation we are to regard the masses $M$ as functions of $\phi$ before taking the $\phi$-derivatives. Thus, when attempting to take $\phi$ derivatives of the second term in Eq. (B7), we should properly go back one step to the original derivation of this term that appears in Eq. (B5) and reinsert a nontrivial mass function $M^{2}(\phi)$ with $\beta_{0}=0$ into the derivation. The remaining derivation of this term then algebraically mirrors the derivation of the massive Bessel-function term in Eq. (B6), only with $M^{2}$ now replaced by $M^{2}(\phi)$ with $\beta_{0}=0$. In other words, for the purposes of our current calculation, we should formally identify

$$
\begin{aligned}
& \frac{\mathcal{M}^{4}}{2} \frac{\pi^{2}}{45}\left(n_{B}-n_{F}\right) a^{3} \\
& \quad=\left.\frac{\mathcal{M}^{2}}{2} \frac{a}{\pi^{2}} \operatorname{Str}_{\beta_{0}=0}\left[M^{2} \sum_{r=1}^{\infty} \frac{1}{r^{2}} K_{2}\left(\frac{r M}{a \mathcal{M}}\right)\right]\right|_{\phi=0}
\end{aligned}
$$

and then evaluate the $\phi$-derivatives before truncating to $\phi=0$. Aside from the overall factor of $-\mathcal{M}^{2} / 2$, acting with $\partial_{\phi}^{2}$ and then truncating to $\phi=0$ yields the same result as on the right side of Eq. (5.14), except with each supertrace over massive states replaced with a supertrace over massless states. We thus need to evaluate these Besselfunction expressions at zero argument. However, for small arguments $z \ll 1$, the Bessel functions have the leading asymptotic behaviors

$$
K_{\nu}(z) \sim \begin{cases}-\log (z / 2)-\gamma+\ldots & \text { for } \nu=0 \\ \frac{1}{2} \Gamma(\nu)(z / 2)^{\nu}+\ldots & \text { for } \nu>0\end{cases}
$$

where $\gamma$ is the Euler-Mascheroni constant. Analyzing the Str $\mathbb{X}_{1}$ term, we thus see that

$$
\begin{aligned}
\frac{2}{\pi} & \lim _{M \rightarrow 0} \underset{M>0}{\operatorname{Str}} \mathbb{X}_{1}\left[\sum_{r=1}^{\infty}\left(\frac{M}{r \mathcal{M}}\right) K_{1}\left(\frac{r M}{a \mathcal{M}}\right)\right] \\
= & \frac{2}{\pi} \operatorname{Str}_{M=0} \mathbb{X}_{1} \lim _{M \rightarrow 0}\left[\sum_{r=1}^{\infty}\left(\frac{M}{r \mathcal{M}}\right)\left(\frac{a \mathcal{M}}{r M}\right)\right] \\
= & \frac{2 a}{\pi} \sum_{r=1}^{\infty} \frac{1}{r^{2}}=\frac{\pi}{3} a,
\end{aligned}
$$

thereby successfully reproducing the corresponding term which appears in $P_{\mathcal{X}}(a)$. Indeed, we see that the $M \rightarrow 0$ limit in Eq. (5.17) is convergent and continuous with the exact $M=0$ result.

For theories in which $\underset{M=0}{\operatorname{Str}} \mathbb{X}_{2}=0$, there are no further terms to consider. The results of this analysis are then clear: within such theories, we have found that

$$
P_{\mathcal{X}}(a)=\left.\partial_{\phi}^{2} P_{\Lambda}(a, \phi)\right|_{\phi=0} .
$$

Through Eq. (5.10), this then implies that

$$
\left.\hat{m}_{\phi}^{2}(\rho, a)\right|_{\mathcal{X}}=\left.\partial_{\phi}^{2} \hat{\Lambda}(\rho, a, \phi)\right|_{\phi=0},
$$


whereupon use of Eq. (4.25) tells us that

$$
\hat{m}_{\phi}^{2}(\rho, a)=\left.\left(\partial_{\phi}^{2}+\frac{\xi}{4 \pi^{2} \mathcal{M}^{2}}\right) \hat{\Lambda}(\rho, a, \phi)\right|_{\phi=0},
$$

or equivalently

$$
\begin{aligned}
\hat{m}_{\phi}^{2}(\mu) & =\left.\left(\partial_{\phi}^{2}+\frac{\xi}{4 \pi^{2} \mathcal{M}^{2}}\right) \hat{\Lambda}(\mu, \phi)\right|_{\phi=0} \\
& =\left.D_{\phi}^{2} \hat{\Lambda}(\mu, \phi)\right|_{\phi=0},
\end{aligned}
$$

where we have defined the modular-covariant derivative

$$
D_{\phi}^{2} \equiv \partial_{\phi}^{2}+\frac{\xi}{4 \pi^{2} \mathcal{M}^{2}}
$$

Of course, for theories with $\underset{M=0}{\operatorname{Str}} \mathbb{X}_{2}=0$, our original unregulated Higgs mass was already finite and a priori there was no need for a regulator. However, even within such theories, it is the use of our modular-invariant regulator for both $\Lambda$ and $m_{\phi}^{2}$ which enabled us to extract EFT descriptions of these quantities and to analyze their runnings as functions of an effective scale $\mu$.

The result in Eq. (5.21) is both simple and profound. Indeed, comparing this result with our starting point in Eq. (2.22) and recalling the subsequent required modular completion in Eq. (2.49), we see that we have in some sense come full circle. However, as stressed above, we have now demonstrated this result using only the general expressions for $\mathbb{X}_{1}$ and $\mathbb{X}_{2}$ in Eq. (5.8) and thus entirely without the assumption of a charge lattice. This result therefore holds for any modular-invariant string theory with $\underset{M=0}{\operatorname{Str}} \mathbb{X}_{2}=0$. Indeed, as indicated above, we can view $D_{\phi}^{2}$ as a modularcovariant derivative, in complete analogy with the latticederived covariant derivative $D_{z}^{2}$ in Eq. (2.41).

But more importantly, we see from Eq. (5.21) that within such theories we can now identify $\hat{\Lambda}(\mu, \phi)$ as an effective potential for the Higgs. Strictly speaking, this is not the entire effective potential-it does not, for example, allow us to survey different minima as a function of $\phi$ in order to select the global and local minima, as would be needed in order to determine the ground states of the theory in different possible phases (with unbroken and/or broken symmetries). However, we see that $\hat{\Lambda}(\mu, \phi)$ does provide a piece of the full potential, namely the portion of the potential in the immediate vicinity of the assumed minimum (around which $\phi$ parametrizes the fluctuations, as always). With this understanding, we shall nevertheless simply refer to $\hat{\Lambda}(\mu, \phi)$ as the Higgs effective potential. Indeed, as expected, we see from Eq. (5.21) that the Higgs mass is related to the curvature of this potential around this minimum. One can even potentially imagine repeating the calculations in this paper without implicitly assuming the stability condition in Eq. (2.50), thereby dropping the implicit assumption that we are sitting at a stable vacuum of the theory. In that case, the first and second $\phi$-derivatives of $\hat{\Lambda}(\mu, \phi)$ would describe the slope and curvature of the potential for arbitrary values of $\phi$, whereupon the methods in this paper could provide a method of "tracing out" the shape of the full potential. However, at best this would appear to be a challenging undertaking.

As remarked above, the form of Eq. (5.21) makes sense from the perspective of Eq. (2.22), in conjunction with the subsequent modular completion. At first glance, it may seem surprising that such a result would continue to survive even after imposing our modular-invariant regulator in order to generate our regulated expressions for $\hat{m}_{\phi}^{2}(\mu)$ and $\hat{\Lambda}(\mu, \phi)$, and perhaps even more surprising after the Rankin-Selberg techniques and their generalizations in Sec. III are employed in order to express these regulated quantities in terms of supertraces over purely physical (level-matched) string states. Ultimately, however, the result in Eq. (5.21) concerns the $\phi$-structure of the theory and the response of the theory to fluctuations in the Higgs field. In theories with $\underset{M=0}{\operatorname{Str}} \mathbb{X}_{2}=0$, these properties are essentially "orthogonal" to the manipulations that occurred in Secs. III and IV, which ultimately concern the regulators and the resulting behavior of these quantities as functions of $\mu$. In other words, in such theories the process of $\phi$-differentiation in some sense "commutes" with all of these other manipulations. Thus the relation in Eq. (5.21) holds not only for our original unregulated Higgs mass and cosmological constant, but also for their regulated counterparts as well as for the running which describes their dependence on the variables defining the regulator.

It is also intriguing that we are able to identify a modularcovariant derivative $D_{\phi}^{2}$ within the results in Eq. (5.21). Of course, this is the second $\phi$-derivative. By contrast, the first $\phi$-derivative does not require modular completion. We have already seen this in Sec. II F, where we found that $\partial_{\phi}$ acting on the partition function $\mathcal{Z}$ corresponds to insertion of the factor $\mathcal{Y}$, which was already modular invariant. In this sense, $\phi$-derivatives are similar to the $z$-derivatives discussed in Sec. II E.

The result in Eq. (5.21) holds only for theories in which $\underset{M=0}{\operatorname{Str}} \mathbb{X}_{2}=0$. However, when $\underset{M=0}{\operatorname{Str}} \mathbb{X}_{2} \neq 0$, there is an additional term to consider within $P_{\Lambda}$. Taking the $M \rightarrow 0$ limit of the $\underset{M>0}{\operatorname{Str}} \mathbb{X}_{2}$ result in Eq. (5.14) in conjunction with the limiting behavior in Eq. (5.16), we formally obtain 


$$
\begin{aligned}
\frac{4}{a} & \lim _{M \rightarrow 0} \operatorname{Str}_{M>0} \mathbb{X}_{2}\left[\sum_{r=1}^{\infty} K_{0}\left(\frac{r M}{a \mathcal{M}}\right)\right] \\
=\frac{4}{a} & \underset{M=0}{\operatorname{Str}} \mathbb{X}_{2} \sum_{r=1}^{\infty}\left[-\log \left(\frac{r M}{2 a \mathcal{M}}\right)-\gamma\right] .
\end{aligned}
$$

Unfortunately, this infinite $r$-summation is not convergent. It also does not correspond to what is presumably the exact $M=0$ result within $P_{\mathcal{X}}$. We stress that these complications arise only when $\underset{M=0}{\operatorname{Str}} \mathbb{X}_{2} \neq 0$, which is precisely the condition under which the original unregulated Higgs mass is divergent.

In order to better understand this phenomenon, we can perform a more sophisticated analysis by analytically performing the $r$-summation in complete generality before taking the $M \rightarrow 0$ limit. We begin by defining the Besselfunction combinations

$$
\mathbb{K}_{\nu}(z) \equiv 2 \sum_{r=1}^{\infty}(r z)^{-\nu} K_{\nu}(r z)
$$

These Bessel-function combinations are relevant for both $P_{\mathcal{X}}$ and $P_{\Lambda}$ in the same way that the combinations $\mathcal{K}_{\nu}^{(n, p)}(z)$ in Eq. (4.36) were relevant for $\left.\hat{m}_{\phi}^{2}\right|_{\mathcal{X}}$ and $\hat{\Lambda}$, and indeed

$$
\mathcal{K}_{\nu}^{(-\nu, p)}(z)=\frac{1}{2}\left[\rho^{-\nu} \mathbb{K}_{\nu}(z / \rho)-\rho^{p} \mathbb{K}_{\nu}(z)\right]
$$

Using the techniques in Ref. [28], it is then straightforward (but exceedingly tedious) to demonstrate that $\mathbb{K}_{\nu}(z)$ for $z \ll 1$ has a Maclaurin-Laurent series representation given by

$$
\begin{aligned}
\mathbb{K}_{\nu}(z)= & \sum_{p=1}^{\nu} 2^{-\nu} \pi^{p} \frac{(-1)^{\nu-p}}{(\nu-p) !} \zeta^{*}(2 p)\left(\frac{z}{2}\right)^{-2 p}+2^{-\nu} \sqrt{\pi} \Gamma\left(\frac{1}{2}-\nu\right) \frac{1}{z} \\
& +\frac{(-2)^{-\nu}}{\nu !}\left[\gamma-\frac{H_{\nu}}{2}+\log (z / 4 \pi)\right]+\sum_{p=1}^{\infty} 2^{-\nu} \pi^{-p} \frac{(-1)^{\nu+p}}{(\nu+p) !} \zeta^{*}(2 p+1)\left(\frac{z}{2}\right)^{2 p}
\end{aligned}
$$

where $H_{n} \equiv \sum_{k=1}^{n} 1 / k$ is the $n$th harmonic number and where $\zeta^{*}(s) \equiv \pi^{-s / 2} \Gamma(s / 2) \zeta(s)=\zeta^{*}(1-s)$ is the "completed" Riemann $\zeta$ function. The representation in Eq. (5.26) is particularly useful for $z \ll 1$, allowing us to extract the leading behaviors

$$
\begin{aligned}
\mathbb{K}_{0}(z) & =\frac{\pi}{z}+\left[\gamma+\log \left(\frac{z}{4 \pi}\right)\right]-\frac{\zeta(3) z^{2}}{8 \pi^{2}}+\frac{3 \zeta(5) z^{4}}{128 \pi^{4}}+\ldots \\
\mathbb{K}_{1}(z) & =\frac{\pi^{2}}{3 z^{2}}-\frac{\pi}{z}-\frac{1}{2}\left[\gamma-\frac{1}{2}+\log \left(\frac{z}{4 \pi}\right)\right]+\frac{\zeta(3) z^{2}}{32 \pi^{2}}-\frac{\zeta(5) z^{4}}{256 \pi^{4}}+\ldots \\
\mathbb{K}_{2}(z) & =\frac{2 \pi^{4}}{45 z^{4}}-\frac{\pi^{2}}{6 z^{2}}+\frac{\pi}{3 z}+\frac{1}{8}\left[\gamma-\frac{3}{4}+\log \left(\frac{z}{4 \pi}\right)\right]-\frac{\zeta(3) z^{2}}{192 \pi^{2}}+\frac{\zeta(5) z^{4}}{2048 \pi^{4}}+\ldots
\end{aligned}
$$

Indeed, use of the expression for $\mathbb{K}_{1}(z)$ confirms our result in Eq. (5.17).

Armed with the expression for $\mathbb{K}_{2}(z)$ in Eq. (5.27), we can now rigorously evaluate the leading terms within $P_{\Lambda}(a)$ - and by extension within $\hat{\Lambda}(\rho, a)$-in complete generality, even when massless states are included. Starting from Eq. (B7) in conjunction with the replacement in Eq. (5.15), we now have

$$
\begin{aligned}
P_{\Lambda}(a) & =\frac{\mathcal{M}^{2}}{24 a} \operatorname{Str} M^{2}-\frac{1}{4 \pi^{2} a} \operatorname{Str} M^{4} \mathbb{K}_{2}\left(\frac{M}{a \mathcal{M}}\right) \\
& \approx \frac{\mathcal{M}^{2}}{24 a} \operatorname{Str} M^{2}-\frac{1}{4 \pi^{2} a} \operatorname{Str}_{0 \leq M \ll a \mathcal{M}} M^{4} \mathbb{K}_{2}\left(\frac{M}{a \mathcal{M}}\right)
\end{aligned}
$$

where the final supertrace on the first line is over all states in the theory, including those that are massless, and where 
in passing to the second line we have recognized that $\mathbb{K}_{2}(z)$ is exponentially suppressed unless $z \ll 1$. The fact that $\mathbb{K}_{2}(z)$ is now explicitly restricted to the $z \ll 1$ regime implies that it is legitimate to insert the series expansion for $\mathbb{K}_{2}(z)$ from Eq. (5.27) within Eq. (5.10). Identifying the physical scale $\mu$ as in Eq. (4.33) and retaining only the leading terms for $\mu \ll M_{s}$, we then obtain

$$
\begin{aligned}
\hat{\Lambda}(\mu, \phi) & =\frac{1}{1+\mu^{2} / M_{s}^{2}}\left\{\frac{1}{24} \mathcal{M}^{2} \operatorname{Str} M^{2}+\underset{0 \leq M \lesssim \mu}{\operatorname{Str}}\left(\frac{M^{2} \mu^{2}}{96 \pi^{2}}-\frac{7 \mu^{4}}{960 \pi^{2}}\right)-\frac{1}{32 \pi^{2}} \underset{0 \leq M \lesssim \mu}{\operatorname{Str}} M^{4} \log \left(\sqrt{2} e^{\gamma+1 / 4} \frac{M}{\mu}\right)+\ldots\right\} \\
& =\frac{1}{24} \mathcal{M}^{2} \operatorname{Str} M^{2}-\operatorname{Str} \frac{M^{2} \mu^{2}}{96 \pi^{2}}+\underset{0 \leq M \lesssim \mu}{\operatorname{Str}}\left(\frac{M^{2} \mu^{2}}{96 \pi^{2}}-\frac{7 \mu^{4}}{960 \pi^{2}}\right)-\frac{1}{32 \pi^{2}} \underset{0 \leq M \lesssim \mu}{\operatorname{Str}} M^{4} \log \left(\sqrt{2} e^{\gamma+1 / 4} \frac{M}{\mu}\right)+\ldots
\end{aligned}
$$

where we have continued to adopt our benchmark value $\rho=2$ and where we recall that each factor of $M$ carries a $\phi$ dependence through Eq. (5.11). Note that in passing to the final expression in Eq. (5.29) we have Taylor-expanded the overall prefactor and kept only those terms of the same order as those already shown. However, we now see that the $\mu^{2}$-term from expanding the prefactor cancels the corresponding $\mu^{2}$-term from $\mathbb{K}_{2}$, leaving behind a net $\mu^{2}$-term which scales as the $M^{2}$ supertrace of only those states whose masses exceed $\mu$. We thus obtain our final result

$$
\begin{aligned}
\hat{\Lambda}(\mu, \phi)= & \frac{1}{24} \mathcal{M}^{2} \operatorname{Str} M^{2}-\underset{M \gtrsim \mu}{\operatorname{Str}} \frac{M^{2} \mu^{2}}{96 \pi^{2}}-\underset{0 \leq M \lesssim \mu}{\operatorname{Str}} \frac{7 \mu^{4}}{960 \pi^{2}} \\
& -\frac{1}{32 \pi^{2}} \underset{0 \leq M \lesssim \mu}{\operatorname{Str}} M^{4} \log \left(\sqrt{2} e^{\gamma+1 / 4} \frac{M}{\mu}\right)+\ldots
\end{aligned}
$$

Indeed, this result provides the leading approximation to the exact expression in Eq. (4.54).

The first and third terms in this result are consistent with those in Eq. (4.56), and indeed the $\mu^{4}$-term is the contribution from the massless states within the second supertrace in Eq. (5.28). However, to this order, we now see that there are two additional terms. The first is a term scaling as $\mu^{2}$ which depends on the spectrum of states with masses $M \gtrsim \mu$. This contribution lies outside the range $M \ll \mu$ studied in Eq. (4.56) but nevertheless generally appears for $M \gtrsim \mu$. The second is a logarithmic term. This term is subleading when compared to the other terms shown, and massless states make no contribution to this term (divergent or otherwise) when evaluated at $\phi=0$ because of its $M^{4}$ prefactor.

This logarithmic term is nevertheless of critical importance when we consider the corresponding Higgs mass. As we have seen in Eq. (5.21), the Higgs mass $\hat{m}_{\phi}^{2}(\mu)$ receives a contribution which scales as $\partial_{\phi}^{2} \hat{\Lambda}(\mu)$. Of course, all of the dependence on $\phi$ is carried within the masses $M$ which appear in Eq. (5.30), and as expected $\hat{\Lambda}(\mu)$ depends not on these masses directly but on their squares. However, for any function $f\left(M^{2}\right)$ we have the algebraic identity

$$
\partial_{\phi}^{2} f\left(M^{2}\right)=\left(\partial_{\phi}^{2} M^{2}\right) \frac{\partial f}{\partial M^{2}}+\left(\partial_{\phi} M^{2}\right)^{2} \frac{\partial^{2} f}{\left(\partial M^{2}\right)^{2}} .
$$

Thus, identifying $f \sim \hat{\Lambda}(\mu)$ and recalling Eq. (5.8), we obtain

$$
\begin{aligned}
\left.\partial_{\phi}^{2} \hat{\Lambda}(\mu)\right|_{\phi=0}= & -\left.4 \pi \operatorname{Str} \mathbb{X}_{1} \frac{\partial \hat{\Lambda}(\mu)}{\partial M^{2}}\right|_{\phi=0} \\
& +\left.16 \pi^{2} \mathcal{M}^{2} \operatorname{Str} \mathbb{X}_{2} \frac{\partial^{2} \hat{\Lambda}(\mu)}{\left(\partial M^{2}\right)^{2}}\right|_{\phi=0}
\end{aligned}
$$

where we have implicitly used the fact that only nonnegative powers of $\phi$ appear within $\hat{\Lambda}(\mu)$, thereby ensuring that our truncation to $\phi=0$ factorizes within each term.

In principle, both supertraces in Eq. (5.32) include massless states. Moreover, we see that the $\operatorname{Str} \mathbb{X}_{1}$ term is proportional to the single $M^{2}$-derivative of $\hat{\Lambda}(\mu)$, and when acting on the logarithm term within Eq. (5.30) we find that massless states continue to be harmless, yielding no contribution (and therefore no divergences). By contrast, we see that the $\operatorname{Str} \mathbb{X}_{2}$ term is proportional to the second $M^{2}$-derivative of $\hat{\Lambda}(\mu)$. This derivative therefore leaves behind a logarithm with no leading $M^{2}$ factors remaining. Thus, for $M=0$, we obtain a logarithmic divergence for the Higgs mass-as expected-so long as $\underset{M=0}{\operatorname{Str}} \mathbb{X}_{2} \neq 0$. Indeed, all of this information is now directly encoded within the effective potential $\hat{\Lambda}(\mu)$ for this theory, as given in Eq. (5.30).

This situation is analogous to the behavior of the traditional Coleman-Weinberg potential $V\left(\varphi_{c}\right)$ as originally given in Refs. $[33,34]$. In that case, it was shown that $V\left(\varphi_{c}\right)$ contains a term scaling as

$$
V\left(\varphi_{c}\right) \sim \varphi_{c}^{4} \log \varphi_{c}^{2}
$$

where $\varphi_{c}$ are the fluctuations of the classical Higgs field around its VEV and where one has assumed a $U(1)$ charged scalar field subject to a $\lambda \phi^{4}$ interaction. The Higgs mass (which goes as the second derivative $\partial^{2} V / \partial \varphi_{c}^{2}$ ) therefore remains finite even as $\varphi_{c} \rightarrow 0$, whereas the fourth 
derivative $\partial^{4} V / \partial \varphi_{c}^{4}$ actually has a logarithmic singularity as $\varphi_{c} \rightarrow 0$. Indeed, this fourth derivative describes the behavior of the coupling $\lambda$. The cure for this disease, as suggested in Refs. [33,34], is to move away from the $\varphi_{c}=0$ origin, and instead define the coupling $\lambda$ at this shifted point.

Of course, in our more general string context, we see that our potential scales like $M^{4} \log M$. Moreover, within the $\mathbb{X}_{2}$ term, it is not the fourth derivative with respect to $M$ which leads to difficulties-rather, it is the second derivative with respect to $M^{2}$. As a consequence, this logarithmic divergence shows up in the Higgs mass rather than in a four-point coupling. That said, it is possible that the cure for this disease may be similar to that discussed in Refs. [33,34]. In particular, this suggests that in string theories for which $\operatorname{Str}_{M=0} \mathbb{X}_{2} \neq 0$, a cure for our logarithmically divergent Higgs mass and the fact that radiative potential is not twice-differentiable there may be similarly found by avoiding the sharp $\phi=0$ truncation that originally appears in Eq. (2.22), and by instead deforming our theory away from the $\phi=0$ origin in $\phi$-space.

Finally, given that we are now equipped with our effective Higgs potential $\hat{\Lambda}(\mu, \phi)$, we can revisit our classical stability condition, as originally discussed in Sec. II F. In general, our theory will be sitting at an extremum of the potential as long as

$$
\left.\partial_{\phi} \hat{\Lambda}(\mu, \phi)\right|_{\phi=0}=0
$$

This, then, is a supplementary condition that we have implicitly assuming to be satisfied within our analysis. Note that

$$
\begin{aligned}
\left.\partial_{\phi} \hat{\Lambda}(\mu, \phi)\right|_{\phi=0} & =\left.\left(\partial_{\phi} M^{2}\right) \frac{\partial \hat{\Lambda}(\mu)}{\partial M^{2}}\right|_{\phi=0} \\
& =\left.4 \pi \mathcal{M} \operatorname{Str} \bigvee \frac{\partial \hat{\Lambda}(\mu)}{\partial M^{2}}\right|_{\phi=0}
\end{aligned}
$$

where

$$
\left.\mathbb{Y} \equiv \frac{1}{4 \pi \mathcal{M}}\left(\partial_{\phi} M^{2}\right)\right|_{\phi=0}
$$

Indeed, for the case of theories with an underlying charge lattice, we have $\mathbb{Y}=\tau_{2}^{-1} \mathcal{Y}$ where $\mathcal{Y}$ is given in Eq. (2.51). Thus the stability of the theory (and the possible existence of a destabilizing $\phi$-tadpole) is closely related to the values of $\mathbb{Y}$ across the string spectrum, as already anticipated in Sec. II F. Substituting the exact expression in Eq. (4.54) into Eq. (5.35), we find

$$
\begin{aligned}
& \left.\partial_{\phi} \hat{\Lambda}(\mu, \phi)\right|_{\phi=0}=\frac{\mathcal{M}^{3}}{1+\mu^{2} / M_{s}^{2}} \operatorname{Str} \mathbb{}\left\{\frac{\pi}{6}+\frac{1}{2 \pi}\left(\frac{M}{\mathcal{M}}\right)^{2}\left[\mathcal{K}_{0}^{(0,1)}\left(\frac{2 \sqrt{2} \pi M}{\mu}\right)+\mathcal{K}_{2}^{(0,1)}\left(\frac{2 \sqrt{2} \pi M}{\mu}\right)\right]\right\} \\
& =\frac{\mathcal{M}^{3}}{1+\mu^{2} / M_{s}^{2}}\left\{\underset{M=0}{\operatorname{Str} Y}\left[\frac{\pi}{6}\left(1+\mu^{2} / M_{s}^{2}\right)\right]\right. \\
& \left.+\underset{M>0}{\operatorname{Str}} \mathbb{Y}\left\{\frac{\pi}{6}+\frac{1}{2 \pi}\left(\frac{M}{\mathcal{M}}\right)^{2}\left[\mathcal{K}_{0}^{(0,1)}\left(\frac{2 \sqrt{2} \pi M}{\mu}\right)+\mathcal{K}_{2}^{(0,1)}\left(\frac{2 \sqrt{2} \pi M}{\mu}\right)\right]\right\}\right\}
\end{aligned}
$$

where in passing to the second expression we have explicitly separated the contributions from the massless and massive string states, and where $\mathcal{K}_{\nu}^{(n, p)}(z)$ continue to denote the combinations of Bessel functions in Eq. (4.36).

Interestingly (but not unexpectedly), the terms multiplying Str $\mathbb{Y}$ in Eq. (5.37) are the same as the terms multiplying Str $\mathbb{X}_{1}$ in Eq. (4.35). Equivalently, we can view the quantity in Eq. (5.37) as the coefficient of the tadpole term (linear in $\phi)$ within the effective potential $\Lambda(\mu, \phi)$ in Eq. (4.54) when the masses are Taylor-expanded as in Eq. (5.11).

There are several ways in which the expressions in Eq. (5.37) might vanish for all $\mu$, as required for a stable vacuum. In principle, for a given value of $\mu$, there might exist a spectrum of states with particular masses $M$ such that the contributions from the Bessel and non-Bessel terms together happen to cancel when tallied across the spectrum.
Any continuous change in the value of $\mu$ might then induce a corresponding continuous change in the spectrum such that this cancellation is maintained. This is not unlike what happens in the traditional field-theoretic ColemanWeinberg potential, where changing the scale $\mu$ can change the vacuum state and the spectrum of excitations built upon it. Of course in the present case we are working within the context of string theory rather than field theory. As such, we are dealing with an infinite tower of string states and simultaneously maintaining modular invariance as the spectrum is deformed.

Another possibility is to simply demand stability in the deep-infrared region, as $\mu \rightarrow 0$. From Eq. (5.37) we see that this would then require simply that

$$
\operatorname{Str} \mathbb{Y}=0
$$


where the supertrace is over all string states, both massless and massive.

A final possibility is to guarantee stability for every value of $\mu$ by demanding the somewhat stronger condition

$\operatorname{Str} \mathbb{Y}=0$ for each mass level individually.

Of course, Eq. (5.39) implies Eq. (5.38), but the fact that Str $\mathbb{Y}$ vanishes for each mass level individually ensures that stability no longer rests on any $\mu$-dependent cancellations involving the Bessel functions.

Comparing Eq. (5.36) with Eq. (5.8), we see that $\mathbb{X}_{2}=\mathbb{Y}^{2}$. However, as discussed below Eq. (2.51), constraints on $\mathbb{Y}$ do not necessarily become constraints on $\mathbb{X}_{2}$, even if the values of $\mathbb{Y}$ happen to cancel pairwise among degenerate states across the string spectrum [which would guarantee Eq. (5.39)]. Thus the requirement of stability does not necessarily lead to any immediate constraints on the supertraces of $\mathbb{X}_{2}$.

In summary, then, we have shown that for theories with $\underset{M=0}{\operatorname{Str}} \mathbb{X}_{2}=0$ there exists an effective Higgs potential $\hat{\Lambda}(\mu, \phi)$ from which the Higgs mass can be obtained through the modular-covariant double derivative $D_{\phi}^{2}$, as in Eq. (5.21). This effective potential is given exactly in Eq. (4.54), with the leading terms given in Eq. (5.30). By contrast, for theories with $\underset{M=0}{\operatorname{Str}} \mathbb{X}_{2} \neq 0$ we have found that the effective potential $\hat{\Lambda}(\mu, \phi)$ picks up an additional contribution whose second derivative is discontinuous at $\phi=0$. In this sense, the Higgs mass is not well defined at $\phi=0$. Of course, one option is to retain the expression obtained in Eq. (4.35); this expression is not the second derivative of $\hat{\Lambda}(\mu, \phi)$ when $\underset{M=0}{\operatorname{Str}} \mathbb{X}_{2} \neq 0$, but it is indeed finite except as $\mu \rightarrow 0$. An alternative option is to define our Higgs mass away from the $\phi=0$ origin. Either way, these features exactly mirror those seen within the traditional Coleman-Weinberg potential.

\section{PULLING IT ALL TOGETHER: DISCUSSION, TOP-DOWN PERSPECTIVES, AND FUTURE DIRECTIONS}

A central question when analyzing any string theory is to understand the properties of its ubiquitous scalars-its Higgs fields, its moduli fields, its axions, and so forth. To a great extent the behavior of a scalar is dominated by its mass, and in this paper we have developed a completely general framework for understanding the masses of such scalars at one-loop order in closed-string theories. Our framework can be applied at all energy scales, is independent of any supersymmetry, and maintains world sheet modular invariance and hence finiteness at all times. Moreover, our framework is entirely string-based and does not rely on establishing any particular low-energy effective field theory. Indeed the notion of an effective field theory at a given energy scale ends up being an output of our analysis, and we have outlined the specific conditions and approximations under which such an EFT emerges from an otherwise completely string-theoretic calculation.

Beyond the crucial role played by the scalar mass, another motivation for studying this quantity is its special status as the "canary in the coal mine" for UV completion. The scalar mass term is virtually the only operator that is both highly UV-sensitive and also IR-divergent when coupled to massless states. Thus, once we understand this operator, we understand much of the entire structure of the theory.

We can appreciate the special status of this operator if we think about a typical EFT. Within such an EFT, the familiar result for the one-loop contributions to the Higgs mass takes the general form

$m_{\phi}^{2}=\frac{M_{\mathrm{UV}}^{2}}{32 \pi^{2}} \underset{\text { eff }}{\operatorname{Str}} \partial_{\phi}^{2} M^{2}-\underset{\text { eff }}{\operatorname{Str}} \partial_{\phi}^{2}\left[\frac{M^{4}}{64 \pi^{2}} \log \left(c \frac{M^{2}}{M_{\mathrm{UV}}^{2}}\right)\right]$

where $M_{\mathrm{UV}}$ is an ultraviolet cutoff, where $c$ is a constant,

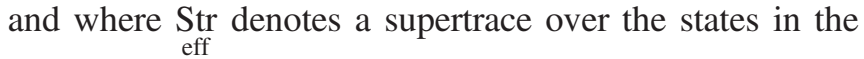
effective theory. This expression has both a quadratic UV-divergence which we would normally subtract by a counterterm as well as a logarithmic cutoff dependence which would normally be indicative of RG running. Thus any UV-completion such as string theory has to resolve two issues within this expression at once: not only must it make the quadratic term finite, but it must also be able to give us specific information about the running. In particular, to what value does the Higgs mass actually run in the IR? Such information is critical in order to nail down the logarithmic running, anchoring it firmly as a function of scale.

Prior to our work, such questions remained unanswered. In retrospect, one clue could already be found in the earlier work of Ref. [18], which in turn rested on previous results in Ref. [16]. In Ref. [18], it was shown that the one-loop cosmological constant $\Lambda$ for any nontachyonic closed string can be expressed as a supertrace over the entire infinite spectrum of level-matched physical string states:

$$
\Lambda=\frac{1}{24} \mathcal{M}^{2} \operatorname{Str} M^{2}
$$

This result, which we have rederived in Eq. (4.45), immediately suggests two things. The first is that it might be possible to derive an analogous spectral supertrace formula for the one-loop Higgs mass within such strings which, like that in Eq. (6.2), depends on only the physical states in the theory. The second, stemming from a comparison between the result in Eq. (6.2) and the first term in Eq. (6.1), is that there might exist a possible derivativebased connection between the one-loop Higgs mass and the one-loop cosmological constant. 
In this study, we have addressed all of these issues. Indeed, one of the central results of our study is an equivalent spectral supertrace formula for the one-loop Higgs mass. Like the calculation of the cosmological constant, our calculation for the Higgs mass relies on nothing more than world sheet modular invariance - an exact symmetry which maintains string finiteness and is preserved, even today. Another of our central results is a deep connection between the Higgs mass and the cosmological constant. However, we also found that unlike the cosmological constant, the Higgs mass may actually have a leading logarithmic divergence. Indeed, this issue depends on the particular string model under study, and in particular the presence of massless states carrying specific charges. As a result of this possible divergence, and as a result of the extreme sensitivity of the Higgs mass to physics at all scales, arriving at a fully consistent treatment of the Higgs mass required us to broach several delicate issues. These encompassed varied aspects of regularization and renormalization and touched on the very legitimacy of extracting an effective field theory from a UV/IR-mixed theory. The scope of our study was therefore quite broad, with a number of important insights and techniques developed along the way.

Our first step was to understand how the Higgs and similar scalars reside within a typical modular-invariant string theory. In particular, for closed-string theories with charge lattices, we began by examining the manner in which fluctuations of the Higgs field deform these charge lattices, all the while bearing in mind that these deformations must preserve modular invariance. We were then able to express the contributions to the Higgs mass in terms of one-loop modular integrals with specific charge insertions $\mathcal{X}_{i}$ incorporated into the string partition-function traces. However, we found that these insertions have an immediate consequence, producing a modular anomaly which then requires us to perform a "modular completion" of the theory. This inevitably introduces an additional term into the Higgs mass, one which is directly related to the oneloop cosmological constant. Our derivation of this term rested solely on considerations of modular invariance and thereby endows this result with a generality that holds across the space of perturbative closed-string models. In this way we arrived at one of the central conclusions of our work, namely the existence of a universal relation between scalar masses and the cosmological constant in any tachyon-free closed-string theory. This relation is given in Eq. (2.55) for four-dimensional theories, and in Eq. (2.56) for theories in arbitrary spacetime dimensions $D$. Stemming only from modular invariance, this result is exact and holds regardless of other dynamics that the theory may experience.

Having established the generic structure of one-loop contributions to the Higgs mass, we then pushed our calculation one step further with the aim of expressing our result for the Higgs mass as a supertrace over the purely physical level-matched spectrum of the theory. Indeed, we demonstrated that the requirements of modular covariance so deeply constrain the contributions to the Higgs mass from the unphysical states that these latter contributions can be expressed in terms of contributions from the physical states alone. However, part of this calculation required dealing with the logarithmic divergences which can arise. This in turn required that we somehow regularize the Higgs mass.

For this reason, we devoted a large portion of our study to establishing a general formalism for regulating quantities such as the Higgs mass that emerge in string theory. We initially considered two forms of what could be called "standard" regulators. The "minimal" regulator is essentially a subtraction of the contributions of the massless states. We referred to this as a minimal regulator because it does not introduce any additional parameters into the theory. Thus, for any divergent quantity, there is a single corresponding regulated quantity. We also discussed what we referred to as a "nonminimal" regulator, based on a mathematical regularization originally introduced in the mathematics literature [13]. This regulator introduces a new dimensionless parameter $t$, so that for any divergent quantity there exist a set of corresponding regularized quantities parametrized by $t$, with the limit $t \rightarrow \infty$ corresponding to the removal of the regulator and the restoration of the original unregulated quantity. This regulator is essentially the one used in Ref. [1] and later in Ref. [27].

As we have explained in Sec. III, both of these regulators yield finite quantities which can be expressed in terms of supertraces over only those string states which are physical (i.e., level-matched). Indeed, in each of these cases, the relation between the regulated quantities and the corresponding supertraces respects modular invariance. Thus, the regulated quantity and the corresponding supertrace in each case transform identically under modular transformations. However, for both the minimal and nonminimal regulators, neither the regulated quantity nor the corresponding supertrace expression is modular invariant on its own. While this additional criterion was not important for the purposes that led to the original development of these regulators in the mathematics literature, this criterion is critical for us because we now wish these regulated quantities to correspond to physical observables (such as our regulated Higgs mass). Each of these regulated quantities must therefore be independently modular invariant on its own.

We therefore presented a third set of regulators-those based on the functions $\hat{\mathcal{G}}_{\rho}(a, \tau)$. These are our modularinvariant regulators, and they depend on two free parameters $(\rho, a)$. Unlike the minimal and nonminimal regulators, these regulators do not operate by performing a sharp, brute-force subtraction of particular contributions within the integrals associated with one-loop string amplitudes. Instead, we simply multiply the integrand of any one-loop 
string amplitude by the regulator function $\hat{\mathcal{G}}_{\rho}(a, \tau)$. These functions have two important properties which make them suitable as regulators when $a \ll 1$. First, as $a \rightarrow 0$, we find that $\hat{\mathcal{G}}_{\rho}(a, \tau) \rightarrow 1$ for all $\tau$. Thus the $a \rightarrow 0$ limit restores our original unregulated theory. Second, $\hat{\mathcal{G}}_{\rho}(a, \tau) \rightarrow 0$ exponentially quickly as $\tau \rightarrow i \infty$ for all $a>0$. These functions thereby suppress all relevant divergences which might appear in this limit. But most importantly for our purposes, $\hat{\mathcal{G}}_{\rho}(a, \tau)$ is completely modular invariant. In particular, this function is completely smooth, with no sharp transitions in its behavior. As a result, multiplying the integrand of any one-loop string amplitude by $\hat{\mathcal{G}}_{\rho}(a, \tau)$ does not simply excise certain problematic contributions within the corresponding string amplitude, but rather provides a smooth, modular-invariant way of deforming (and thereby regulating) the entire theory. This function even has a physical interpretation in the $\rho=2$ special case, arising as the result of the geometric deformations discussed in Refs. [4-6].

Armed with this regulator, we then demonstrated that our regulated Higgs mass can be expressed as the supertrace over only the physical string states. Our result for $\hat{m}_{\phi}^{2}(\rho, a)$ is given in Eq. (4.25), where $\left.\hat{m}_{\phi}^{2}(\rho, a)\right|_{\mathcal{X}}$ is given in Eq. (A16) and where $\hat{\Lambda}(\rho, a)$ is given in Eq. (B8). We stress that this is the exact string-theoretic result for the regulated Higgs mass, expressed as a function of the regulator parameters $(\rho, a)$. Moreover $\hat{\Lambda}(\rho, a)$ by itself is the corresponding regulated cosmological constant. As discussed in the text, the one-loop cosmological constant $\Lambda$ requires regularization in this context even though it is already finite in all tachyon-free closed-string theories.

We originally derived these results under the assumption that our underlying string theory could be formulated with an associated charge lattice. This assumption gave our calculations a certain concreteness, allowing us to see exactly which states with which kinds of charges ultimately contribute to the Higgs mass. However, we then proceeded to demonstrate that many of our results are actually more general than this, and do not require a charge lattice at all. This lattice-free reformulation also had an added benefit, allowing us to demonstrate a second deep connection between the Higgs mass and the cosmological constant beyond that in Eq. (2.55). In particular, we were able to demonstrate that each of these quantities can be expressed in terms of a common underlying quantity $\hat{\Lambda}(\rho, a, \phi)$ via relations of the form

$$
\left\{\begin{array}{l}
\hat{\Lambda}(\rho, a)=\left.\hat{\Lambda}(\rho, a, \phi)\right|_{\phi=0}, \\
\hat{m}_{\phi}^{2}(\rho, a)=\left.D_{\phi}^{2} \hat{\Lambda}(\rho, a, \phi)\right|_{\phi=0}
\end{array}\right.
$$

where $D_{\phi}^{2}$ is the modular-covariant second $\phi$-derivative given in Eq. (5.22). These relations allow us to interpret $\hat{\Lambda}(\rho, a, \phi)$ as a stringy effective potential for the Higgs.
Indeed, these relations are ultimately the fulfillment of our original suspicion that the Higgs mass might be related to the cosmological constant through a double $\phi$-derivative, as discussed below Eq. (6.2). However, we now see from Eq. (5.22) that this is not just an ordinary $\phi$-derivative $\partial_{\phi}^{2}$, but rather a modular-covariant derivative, complete with anomaly term. The second relation in Eq. (6.3) thereby subsumes our original relation between the Higgs mass and the cosmological constant, as expressed in Eq. (2.55). Moreover, we see that the regulated cosmological constant $\hat{\Lambda}(\rho, a)$ is nothing but the $\phi=0$ truncation of the same effective potential $\hat{\Lambda}(\rho, a, \phi)$. In this way, $\hat{\Lambda}(\rho, a, \phi)$ emerges as the central object from which our other relevant quantities can be obtained.

At no step in the derivation of these results was modular invariance broken. Thus all of these results are completely consistent with modular invariance, as required. Moreover, expressed as functions of the world sheet regulator parameters $(\rho, a)$, all of our quantities are purely string-theoretic and there are no ambiguities in their definitions.

Our next goal was to interpret these regulated quantities in terms of a physical cutoff scale $\mu$. Of course, if we had been working within a field-theoretic context, all of our regulator parameters would have had direct spacetime interpretations in terms of a spacetime scale $\mu$. As a result, varying the values of these regulator parameters would have led us directly to a renormalization-group flow with an associated RGE. String theories, by contrast, are formulated not in spacetime but on the world sheet-for such strings, spacetime is nothing but a derived quantity. As a result, although we were able to express our regulated quantities as functions of the two regulator parameters $(\rho, a)$, the only way to extract an EFT description from these otherwise complete string-theoretic expressions was to develop a mapping between the world sheet parameters $(\rho, a)$ and a physical spacetime scale $\mu$.

As we have seen, this issue of connecting $(\rho, a)$ to $\mu$ is surprisingly subtle, and it is at this step that we must make certain choices that break modular invariance. We already discussed some the issues surrounding IR/UV equivalence in Sec. IVA-indeed, these issues already suggested that the passage to an EFT would be highly nontrivial and involve the breaking of modular invariance. But now, with our complete results in hand, we can take a bird's-eye view and finally map out the full structure of the problem.

Our understanding of this issue is summarized in Fig. 4. Although the specific situation sketched in Fig. 4 corresponds to our modular-invariant regulator function $\hat{\mathcal{G}}_{\rho}(a, \tau)$, the structure of this diagram is general. Ultimately, the connection between world sheet physics and spacetime physics follows from the one-loop partition function, which for physical string states of spacetime masses $M_{i}$ takes the general form 


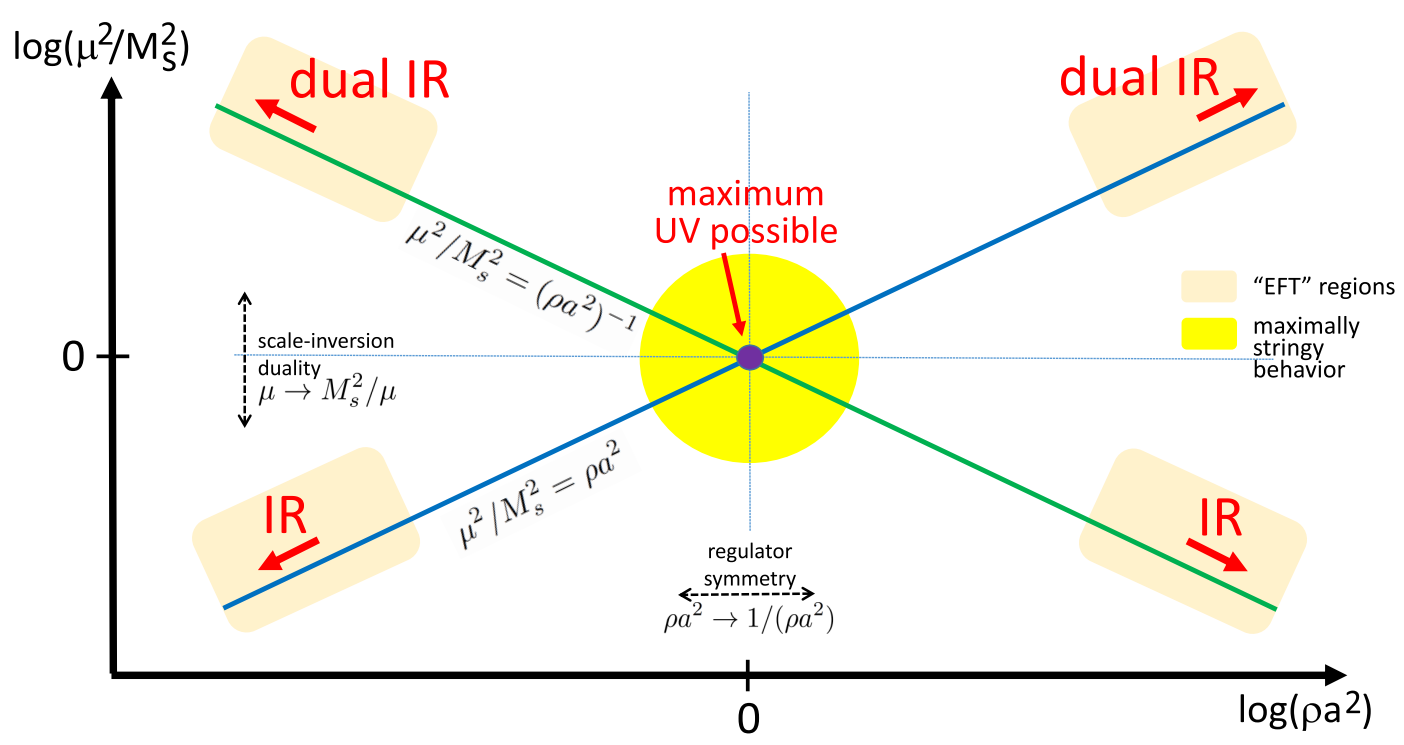

FIG. 4. The scale structure of physical quantities in a modular-invariant string theory. For the modular-invariant regulator function $\hat{\mathcal{G}}_{\rho}\left(a, \tau_{2}\right)$ discussed in this paper, the mapping between the regulator parameter $\rho a^{2}$ and the physical spacetime scale $\mu$ has two distinct branches. The traditional branch (shown in blue) identifies $\mu^{2} / M_{s}^{2}=\rho a^{2}$, but modular invariance implies the existence of an invariance under the scale-inversion duality symmetry $\mu \rightarrow M_{s}^{2} / \mu$. This in turn implies the existence of a second branch (shown in green) on which we can alternatively identify $\mu^{2} / M_{s}^{2}=1 /\left(\rho a^{2}\right)$. Although $\hat{\mathcal{G}}_{\rho}\left(a, \tau_{2}\right)$ functions as a regulator for $\rho a^{2}<1$, its symmetry under $\rho a^{2} \rightarrow$ $1 /\left(\rho a^{2}\right)$ implies that this function also acts as a regulator when extended into the $\rho a^{2}>1$ region. This then allows us to see the full fourfold modular structure of the theory. The Higgs-mass plot shown in Fig. 3 can now be understood as following the $\mu^{2} / M_{s}^{2}=\rho a^{2}$ branch from the lower-left corner of this figure inward toward the central location at which $\mu=M_{s}$, after which it then follows the $\mu^{2} / M_{s}^{2}=1 /\left(\rho a^{2}\right)$ branch outward toward the upper-left corner. However, in a modular-invariant theory, all four quadrants of this figure are equivalent and describe the same physics. Likewise, in such theories there is no distinction between IR and UV. Thus one can exchange "IR" ↔ "UV" within all labels of this sketch, and we have simply chosen to show those labels that have the most natural interpretations within the lower-left quadrant. Finally, regions with beige shading indicate locations where EFT descriptions exist, while stringy effects dominate in the yellow central region. As a result, focusing on any one of the four EFT regions by itself necessarily breaks modular invariance because the choice of EFT region is tantamount to picking a preferred direction for the flow of the scale $\mu$ relative to the underlying string-theoretic regulator parameter $\rho a^{2}$. However, even within the EFT regions, string states at all mass scales contribute nontrivially. Thus even these EFT regions differ from what might be expected within quantum field theory.

$$
\mathcal{Z} \sim \tau_{2}^{-1} \sum_{i} e^{-\pi \alpha^{\prime} M_{i}^{2} \tau_{2}}
$$

These $M_{i}$ are precisely the masses which ultimately appear in our physical supertrace formulas. However, our regulator function $\hat{\mathcal{G}}_{\rho}(a, \tau)$ cannot regulate the divergences that might arise from light and/or massless states as $\tau \rightarrow i \infty$ unless it suppresses contributions to the partition function within the region $\tau_{2} \gtrsim \tau_{2}^{*}$ for some $\tau_{2}^{*}$. We thus see that whether a given state contributes significantly to one-loop amplitudes in the presence of the regulator depends on the magnitude of $\alpha^{\prime} M_{i}^{2} \tau_{2}^{*}$. This immediately leads us to identify a corresponding spacetime physical RG scale $\mu^{2} \sim 1 /\left(\alpha^{\prime} \tau_{2}^{*}\right)$. Indeed, this was precisely the logic that originally led us to Eq. (4.21). Moreover, for our specific regulator function $\hat{\mathcal{G}}_{\rho}(a, \tau)$, we have $\tau_{2}^{*} \sim 1 /\left(\rho a^{2}\right)$, thus leading to the natural identification $\mu^{2} / M_{s}^{2}=\rho a^{2}$.

However, modular invariance does not permit us to identify just one special point $\tau_{2}=\tau_{2}^{*}$ along the $\tau_{1}=0$ axis within the fundamental domain. Indeed, for every such special point, the corresponding point with $\tau_{2}=1 / \tau_{2}^{*}$ is equally special, since these two points along the $\tau_{1}=0$ axis are related by the $\tau \rightarrow-1 / \tau$ modular transformation. In other words, although our $\hat{\mathcal{G}}_{\rho}(a, \tau)$ regulator function suppresses contributions from the $\tau_{2} \gtrsim \tau_{2}^{*}$ region, the modular invariance of this function requires that it also simultaneously suppress contributions from the region with $\tau_{2} \lesssim 1 / \tau_{2}^{*}$. (In general a modular-invariant regulator function equally suppresses the contributions from the regions that approach any of the modular cusps, but for the purposes of mapping to a physical spacetime scale $\mu$ our concern is limited to the cusps along the $\tau_{1}=0$ axis.) We thus see that for any value of the spacetime scale $\mu$ that we attempt to identify as corresponding to $\rho a^{2}$, there always exists a second scale $M_{s}^{2} / \mu$ which we might equally validly identify as corresponding to $\rho a^{2}$. This is the implication of the $\mu \rightarrow M_{s}^{2} / \mu$ scale-inversion symmetry discussed in Sec. IV.

The upshot of this discussion is that the mapping from $\rho a^{2}$ to $\mu$ in any modular-invariant theory actually has two 
branches, as shown in Fig. 4. Along the first branch we identify $\mu^{2} / M_{s}^{2}=1 / \tau_{2}^{*}=\rho a^{2}$, but along the second branch we identify $\mu^{2} / M_{s}^{2}=\tau_{2}^{*}=1 /\left(\rho a^{2}\right)$. These branches contain the same physics, but the choice of either branch breaks modular invariance. In this respect modular invariance is much like another description-redundancy symmetry, namely gauge invariance: all physical quantities must be gauge invariant, but the choice of a particular gauge slice (which is tantamount to the choice of a particular branch) necessarily breaks the underlying symmetry.

In most of our discussions in this paper, we focused on the behavior of our regulator functions within the $a \ll 1$ regime. However, as we have seen in Eq. (3.40), these functions exhibit a symmetry under $\rho a^{2} \rightarrow 1 /\left(\rho a^{2}\right)$. The logical necessity for this extra symmetry will be discussed below, but this symmetry implies that $\hat{\mathcal{G}}_{\rho}(a, \tau)$ also acts as a regulator when extended into the $a \gg 1$ region. This then allows us to see the full fourfold modular structure of the theory, as shown in Fig. 4. Given this structure, we can also revisit the Higgs-mass plot shown in Fig. 3. We now see that we can interpret this plot as following the $\mu^{2} / M_{s}^{2}=\rho a^{2}$ branch from the lower-left corner of Fig. 4 toward the central location at which $\mu=M_{s}$, and then following the $\mu^{2} / M_{s}^{2}=1 /\left(\rho a^{2}\right)$ branch outward toward the upper-left corner.

Given the sketch in Fig. 4, we can also understand more precisely how the passage from string theory to an EFT breaks modular invariance. Within this sketch, regions with beige shading indicate locations where EFT descriptions exist (and where our regulators are designed to function most effectively, with $a \ll 1$ or $a \gg 1$ ). By contrast, stringy effects dominate in the yellow central region, which is the only region that locally exhibits the full modular symmetry, lying on both branches simultaneously. As a result, we necessarily break modular invariance by choosing to focus on any one of the four EFT regions alone. Indeed, each EFT region intrinsically exhibits a certain direction for the flow of the scale $\mu$ relative to the flow of the underlying world sheet parameter $\rho a^{2}$. However, the relative direction of this flow is not modular invariant, as evidenced from the fact that this flow is reversed in switching from one branch to the other.

At first glance, the fact that the EFT regions appear only at the extreme ends of each branch in Fig. 4 might lead one to believe that only extremely light states contribute within the EFT and that the infinite towers of heavy string states can be ignored within such regions. However, as we have repeatedly stressed throughout this paper, even this seemingly mild assertion would be incorrect. For example, even within the $\mu \rightarrow 0$ limit, we have seen in Eq. (4.58) that the Higgs mass receives contributions from $\mathbb{X}_{1}$-charged states of all masses across the entire string spectrum. Likewise, $\Lambda$ receives contributions from all string states, regardless of their mass. We have also seen that the Higgs mass accrues a $\mu$-dependence which transcends our fieldtheoretic expectations, even for $\mu \ll M_{s}$. A particularly vivid example of this is the unexpected "dip" region shown in Fig. 3-an effect which is the direct consequence of the stringy Bessel functions whose form is dictated by modular invariance. Thus modular invariance continues to govern the behavior of the Higgs mass at all scales, even within the EFT regions.

Likewise, within such theories there is no distinction between IR and UV. We can already see this within Fig. 4, where the points near the upper end of the figure (i.e., with large $\mu$ ) are designated not as "UV" but as "dual IR," since they are the images of the IR regions with small $\mu$ under the duality-inversion symmetry. But even this labeling is not truly consistent with modular invariance, since there is no reason to adopt the language of the small- $\mu$ region in asserting that the bottom part of the figure corresponds to the IR. Thanks to the equivalence under $\mu \rightarrow M_{s}^{2} / \mu$, we might as well have decided to label the upper portion of the figure as "UV" and the lower portion of the figure as "dual UV." In that case, the center of the figure would represent the most IR behavior that is possible, rather than the most UV. The upshot is that the mere distinction between "IR" and "UV" itself breaks modular invariance. In a modularinvariant theory, what we would normally call a UV divergence is not distinct from an IR divergence-they are one and the same. Indeed, we have seen that the quadratic divergences normally associated with the Higgs mass in field theory are softened to mere logarithmic divergences - such as the power of modular invariancebut in string theory there is no deeper physical interpretation to this remaining divergence as either UV or IR in nature until we decide to introduce one.

In this connection, we note that it might have seemed tempting to look at the EFT expression in Eq. (6.1) and suppose that in a UV-complete theory one could have set about the calculation in a piecemeal manner, dividing the contributions into a UV contribution and a much less lethal logarithmically divergent IR contribution and then evaluating each one separately. This is certainly the kind of reasoning that is suggested by the notion of softly broken symmetries, for example. However, because there is no intrinsic notion of UV and IR in a modular-invariant theory, no such separation can exist. Instead, all we have in string theory are amplitudes which may be divergent, and the question as to whether such divergences are most naturally interpreted as UV or IR in nature ultimately boils down to a convention as to which modular-group fundamental domain is selected as our region of integration. Although these arguments are expressed in terms of one-loop amplitudes, similar arguments extend to higher loops as well. Of course, most standard textbook recipes for evaluating one-loop modular integrals in string theory adopt the fundamental domain which includes the cusp at $\tau \rightarrow i \infty$. This choice then leads to an IR interpretation for 
the divergence. But when we derived our supertrace expressions involving only the physical string states, our calculations required that we sum over an infinite number of such fundamental domains which are all related to each other under modular transformations, as in Eq. (3.6). It is only in this way that we were able to transition from the fundamental domain to the strip and thereby obtain supertraces involving only the physical string states. Thus UV and IR physics are inextricably mixed within such supertrace expressions.

Given this bird's-eye view, we can now also understand in a deeper way why it was necessary for us to switch from our original modular-invariant regulator functions $\mathcal{G}_{\rho}(a, \tau)$ in Eq. (3.32) to our enhanced modular-invariant functions $\hat{\mathcal{G}}_{\rho}(a, \tau)$ in Eq. (3.39) which exhibited the additional symmetry under $a \rightarrow 1 /(\rho a)$. At the level of the string world sheet, our original functions $\mathcal{G}_{\rho}(a, \tau)$ would have been suitable, since they already satisfied the two critical criteria which made them suitable as regulators:

- $\mathcal{G}_{\rho}(a, \tau) \rightarrow 1$ for all $\tau$ as $a \rightarrow 0$, so that the $a \rightarrow 0$ limit restores our original unregulated theory; and

- $\mathcal{G}_{\rho}(a, \tau) \rightarrow 0$ sufficiently rapidly for any $a>0$ as $\tau_{2}$ approaches the appropriate cusps ( $\tau \rightarrow i \infty$, or equivalently $\tau \rightarrow 0$ ), so that $f$ is capable of regulating our otherwise-divergent integrands for all $a>0$.

Indeed, for any divergent string-theoretic quantity $I$, these functions would have led to a corresponding set of finite quantities $\tilde{I}_{\rho}(a)$ for each value of $(\rho, a)$. We further saw that these $\mathcal{G}$ functions had a redundancy under $(\rho, a) \rightarrow(1 / \rho, \rho a)$, so that the only the combination $\rho a^{2}$ was invariant.

However, while such functions would have been suitable at the level of the string world sheet, there is one additional condition that must also be satisfied if we want to be able to interpret our results in spacetime, with the invariant combination $\rho a^{2}$ identified as a running spacetime scale $\mu^{2} / M_{s}^{2}$. As we have argued below Eq. (6.4), modularinvariant string theories necessarily exhibit an invariance under $\mu \rightarrow M_{s}^{2} / \mu$; indeed, this scale-duality symmetry rests on very solid foundations. However, given this scaleinversion symmetry, we see that we would not have been able to consistently identify $\rho a^{2}$ with the spacetime scale $\mu^{2} / M_{s}^{2}$ unless our regulator function itself also exhibited such an inversion symmetry, with an invariance under $\rho a^{2} \rightarrow 1 /\left(\rho a^{2}\right)$ [or equivalently under $a \rightarrow 1 /(\rho a)$ ]. This was the ultimately the reason we transitioned from the $\mathcal{G}$ functions to the $\hat{\mathcal{G}}$ functions, as in Eq. (3.39). This not only preserved the first two properties itemized above, but also ensured a third:

- $\hat{\mathcal{G}}_{\rho}(a, \tau)=\hat{\mathcal{G}}_{\rho}(1 / \rho a, \tau)$ for all $(\rho, a)$.

In other words, while our first two conditions ensured proper behavior for our regulator functions on the string world sheet, it was the third condition which allowed us to endow our regulated string theory with an interpretation in terms of a renormalization flow with a spacetime mass scale $\mu$. Indeed, we see from Fig. 4 that in some sense this extra symmetry was forced on us the moment we identified $\mu^{2} / M_{s}^{2}=\rho a^{2}$ and recognized the existence of the scaleduality symmetry under $\mu \rightarrow M_{s}^{2} / \mu$. A similar symmetry structure would also need to hold for any alternative regulator functions that might be chosen.

Given these insights, we then proceeded to derive expressions for our regulated Higgs mass $\hat{m}_{\phi}^{2}(\mu)$ and regulated cosmological constant (effective potential) $\hat{\Lambda}(\mu)$ as functions of $\mu$. The exact results for these quantities are given in Eqs. (4.35) and (4.54), respectively. Once again, we stress that these results are fully modular invariant except for the fact that we have implicitly chosen to work within the lower-left branch of Fig. 4. For $\mu \ll M_{s}$, we were then able to derive the corresponding approximate EFT running for these quantities in Eqs. (4.42) and (4.56). Indeed, as we have seen in Eq. (5.30), our final result for the running effective potential $\hat{\Lambda}(\mu, \phi)$ takes the general form

$$
\begin{aligned}
\hat{\Lambda}(\mu, \phi)= & \frac{1}{24} \mathcal{M}^{2} \operatorname{Str} M^{2}-c^{\prime} \underset{M \gtrsim \mu}{\operatorname{Str}} M^{2} \mu^{2} \\
& -\underset{0 \leq M \lesssim \mu}{\operatorname{Str}}\left[\frac{M^{4}}{64 \pi^{2}} \log \left(c \frac{M^{2}}{\mu^{2}}\right)+c^{\prime \prime} \mu^{4}\right]
\end{aligned}
$$

where $c=2 e^{2 \gamma+1 / 2}, c^{\prime}=1 /\left(96 \pi^{2}\right)$, and $c^{\prime \prime}=7 c^{\prime} / 10$, and where of course we regard the masses $M^{2}$ as a functions of $\phi$ as in Eq. (5.11). These specific values of $\left\{c, c^{\prime}, c^{\prime \prime}\right\}$ were of course calculated with our regulator function taken as $\hat{\mathcal{G}}_{\rho}(a, \tau)$ assuming the benchmark value $\rho=2$, and with $\mu$ defined along the lower-left branch in Fig. 4. However, in general these constants depend on the precise profile of our regulator function. Finally, given our effective potential, we also discussed the general conditions under which our theory is indeed sitting at a stable minimum as a function of $\phi$.

With the results in Eq. (6.5) in conjunction with the relations in Eq. (6.3), we have now obtained an understanding of the Higgs mass as emerging from $\phi$-derivatives of an infinite spectral supertrace of regulated effective potentials. We can now also perceive the critical similarities and differences relative to the EFT expectations in Eq. (6.1) and thereby address the questions posed at the beginning of this section. For example, from the first term within Eq. (6.5) we see that the Higgs mass within the full modular-invariant theory contains a term of the form $\frac{1}{24} \mathcal{M}^{2} \operatorname{Str} \partial_{\phi} M^{2}$. Comparing this term with first term within Eq. (6.1), we might be tempted to identify $M_{\mathrm{UV}}=$ $\sqrt{3 / 2} \pi \mathcal{M}$. However, despite the superficial resemblance between these terms, we see that the full string-theoretic term is very different because the relevant supertrace is over the entire spectrum of states in the theory and not just the light states in the EFT. 
It is also possible to compare the logarithmic terms within Eqs. (6.1) and (6.5). Of course, as in the standard treatment, the logarithmic term in Eq. (6.1) can be regulated by subtracting a term of the form $\log \left(M_{\mathrm{UV}} / \mu\right)$, thereby obtaining an effective running. We then see that both logarithmic terms actually agree. While it is satisfying to see this agreement, it is nevertheless remarkable that we have obtained such a logarithmic EFT-like running from our string-theoretic result. As we have seen, our full string results in Eqs. (4.35) and (4.54) did not contain logarithms-they contained Bessel functions. Moreover, unlike the term discussed above, their contributions were not truncated to only the light states with $M \lesssim \mu$-they involved supertraces over all of the states in the string spectrum, as expected for a modular-invariant theory. However, the behavior of the Bessel functions themselves smoothly and automatically suppressed the contributions from states with $M \gtrsim \mu$. Thus, we did not need to impose the $M \lesssim \mu$ restriction on the supertrace of the logarithm term in Eq. (6.5) based on a prior EFT-based expectation, as in Eq. (6.1); this restriction, and thus an EFT-like interpretation, emerged naturally from the Bessel functions themselves. It is, of course, possible to verify the appearance of such a term directly within the context of a given compactification through a direct calculation of the twopoint function of the Higgs field (and indeed we verified this explicitly for various compactification choices), but of course the expression in Eq. (6.5) is completely general and thus holds regardless of the specific compactification.

We can also now answer the final question posed at the beginning of this section: to what value does the Higgs mass actually run as $\mu \rightarrow 0$ ? Assuming $\underset{M=0}{\operatorname{Str}} \mathbb{X}_{2}=0$, the answer is clear from Eq. (4.58):

$$
\begin{aligned}
\lim _{\mu \rightarrow 0} \hat{m}_{\phi}^{2}(\mu) & =\frac{\xi}{4 \pi^{2}} \frac{\Lambda}{\mathcal{M}^{2}}-\frac{\pi}{6} \mathcal{M}^{2} \operatorname{Str} \mathbb{X}_{1} \\
& =\frac{\xi}{96 \pi^{2}} \operatorname{Str} M^{2}+\left.\frac{1}{24} \mathcal{M}^{2} \operatorname{Str} \partial_{\phi}^{2} M^{2}\right|_{\phi=0} \\
& =\left.\frac{\mathcal{M}^{2}}{24} D_{\phi}^{2} \operatorname{Str} M^{2}\right|_{\phi=0} .
\end{aligned}
$$

From a field-theory perspective, this is a remarkable result: all running actually stops as $\mu \rightarrow 0$, and the Higgs mass approaches a constant whose value is set by a supertrace over all of the states in the string spectrum. This behavior is clearly not EFT-like. However, the underlying reason for this has to do with UV/IR equivalence and the scale-inversion symmetry under $\mu \rightarrow M_{s}^{2} / \mu$. Regulating our Higgs mass ensures that our theory no longer diverges as $\mu \rightarrow \infty$; rather, the Higgs mass essentially "freezes" to a constant in this limit. It is of course natural that in this limit the relevant constant includes contributions from all of the string states. The scale-inversion symmetry then implies that the Higgs mass must also "freeze" to exactly the same value as $\mu \rightarrow 0$. We thus see that although a portion of the running of the Higgs mass is EFT-like when $\mu \ll M_{s}$, this EFT-like behavior does not persist all the way to $\mu=0$ because the scale-inversion symmetry forces the behavior as $\mu \rightarrow 0$ to mirror the behavior as $\mu \rightarrow \infty$. Indeed, the "dip" region is nothing but the stringy transition between these two regimes.

Given the results in Eq. (6.6), we also observe that we can now write

$$
m_{\phi}^{2}=\left.\frac{1}{24} \mathcal{M}^{2} \operatorname{Str}\left[D_{\phi}^{2} M^{2}(\phi)\right]\right|_{\phi=0} .
$$

This result is thus the Higgs-mass analogue of the $\Lambda$-result in Eq. (6.2).

The final results of our analysis are encapsulated within Fig. 3. Indeed, this figure graphically illustrates many of the most important conclusions of this paper. In Fig. 3, we have dissected the anatomy of the Higgs-mass running, illustrating how this running passes through different distinct stages as $\mu$ increases. Starting from the "deep IR/UV" region near $\mu \approx 0$, the Higgs mass passes through the "dip" region and the "EFT" region before ultimately reaching the "turnaround" region. Beyond this, the theory enters the "dual EFT" region, followed by the "dual dip" region and ultimately the "dual deep-IR/UV" region. Above all else, this figure clearly illustrates how in a modular-invariant theory our normal understanding of "running" is turned on its head. The Higgs mass does not somehow get "born" in the UV and then run to some possibly undesirable value in the IR. Instead, we may more properly consider the Higgs mass to be "born" at $\mu=M_{s}$. It then runs symmetrically toward both lesser and greater values of $\mu$ until it eventually asymptotes to a constant as $\mu \rightarrow 0$ and as $\mu \rightarrow \infty$.

We conclude this discussion with two comments regarding technical points. First, as discussed in Sec. IV, we have freely assumed throughout this paper that the residue of a supertrace sum is equivalent to the supertrace sum of the individual residues. In other words, as discussed below Eq. (4.15), we have assumed that the supertrace sum does not introduce any additional divergences beyond those already encapsulated within our assertion that the fourdimensional Higgs mass is at most logarithmically divergent, or equivalently that the level-matched integrand has a divergence structure $g(\tau) \sim c_{0}+c_{1} \tau_{2}$ as $\tau_{2} \rightarrow \infty$. Indeed, this assumption is justified because we are working within the presence of a regulator which is sufficiently powerful to render our modular integrals finite, given this divergence structure. Moreover, the divergence structure of our original unregulated Higgs mass is completely general for theories in four spacetime dimensions, since only a change in spacetime dimension can alter the numbers of $\tau_{2}$ prefactors which emerge. Of course, four-dimensional string models generically contain many moduli, and some of these moduli may correspond to the radii associated with possible 
geometric compactifications from our original underlying 10- and/or 26-dimensional world sheet theories. If those moduli are extremely large or small, one approaches a decompactification limit in which our theory becomes effectively higher-dimensional. For any finite or nonzero value of these moduli, our results still hold as before. However, in the full limit as these moduli become infinite or zero, new divergences may appear which are related to the fact that the effective dimensionality of the theory has changed. Indeed, extra spacetime dimensions generally correspond to extra factors of $\tau_{2}$, thereby increasing the strengths of the potential divergences. Although all of our results in Secs. II and III are completely general for all spacetime dimensions, our results in Sec. IV are focused on the case of four-dimensional string models for which $g\left(\tau_{2}\right) \sim$ $c_{0}+c_{1} \tau_{2}$ as $\tau_{2} \rightarrow \infty$. As a result, the supertrace-summation and residue-extraction procedures will not commute in the decompactification limit, and additional divergences can arise. However, this does not pose a problem for us-we simply use the same regulators we have already outlined in Sec. III, but instead work directly in a higher-dimensional framework in which $g\left(\tau_{2}\right)$ as $\tau_{2} \rightarrow \infty$ takes a form appropriate for the new effective spacetime dimensionality. Once this is done, we are once again free to exchange the orders of residue extraction and supertrace summation, knowing that our results must once again be finite.

Our second technical point relates to the concern that has occasionally been expressed in the prior literature about the role played by the off-shell tachyons which necessarily appear within the spectra of all heterotic strings, and the exponential one-loop divergences they might seem to induce in the absence of supersymmetry as $\tau \rightarrow i \infty$. In this paper, we discussed this issue briefly in the paragraph surrounding Eq. (4.7). Ultimately, however, we believe that this concern is spurious. First, as discussed below Eq. (4.7), such states typically lack the nonzero charges needed in order to contribute to the relevant one-loop string amplitudes. Second, within such one-loop amplitudes, our modular integrations come with an implicit instruction that within the $\tau_{2}>1$ region of the fundamental domain we are to perform the $\tau_{1}$ integration prior to performing the $\tau_{2}$ integration. This then eliminates the contributions from the off-shell tachyons in the $\tau \rightarrow i \infty$ limit. This integrationordering prescription is tantamount to replacing the divergence as $\tau \rightarrow i \infty$ with its average along the line segment $-1 / 2 \leq \tau_{1} \leq 1 / 2$, which makes sense in the $\tau_{2} \rightarrow \infty$ limit as this line segment moves infinitely far up the fundamental domain. Another way to understand this is to realize that under a modular transformation no information can be lost, yet this entire line segment as $\tau_{2} \rightarrow \infty$ is mapped to the single point with $\tau_{1}=\tau_{2}=0$ under the modular transformation $\tau \rightarrow-1 / \tau$. Finally, through the compactification/ decompactification argument presented in Ref. [16], one can see directly that this off-shell tachyon makes no contribution in all spacetime dimensions $D>2$. Thus no exponential divergence arises. However, we note that even if an exponential divergence were to survive, it would also be automatically regulated through our modular-invariant regulator $\hat{G}_{\rho}(a, \tau)$-or sufficiently many higher powers thereof-given that $\hat{G}_{\rho}(a, \tau)$ itself exhibits an exponential suppression as $\tau \rightarrow i \infty$.

The results in this paper have touched on many different topics. Accordingly, there are several directions that future work may take.

First, although we have focused in this paper on the mass of the Higgs, it is clear that this UV/IR-mixed picture of running provides a general paradigm for how one should think about the behavior of a modular-invariant theory as a whole. For example, one question that naturally arises from our discussion concerns the renormalization of the dimensionless couplings. This was the subject of the seminal work in Ref. [1]. Even though a regulator was chosen in Ref. [1] which was not consistent with modular invariance, this was one of the first calculations in which the contributions from the full infinite towers of string states were incorporated within a calculation of gauge couplings and their behavior. It would therefore be interesting to revisit these issues and analyze the running and beta functions of the dimensionless gauge couplings that would emerge in the presence of a fully modular-invariant regulator. The first steps in this direction have already been taken in Refs. [4-6]. However, using the techniques we have developed in this paper, it is now possible to extend these results to obtain full scaledependent RG flows for the gauge couplings as functions of $\mu$, and in a continuous way that simultaneously incorporates both UV and IR physics and which does not artificially separate the results into a field-theoretic running with a string-theoretic threshold correction. Moreover, due to the $\mu \rightarrow M_{s}^{2} / \mu$ symmetry we expect that the coefficients of all operators in the theory should experience symmetric runnings with vanishing gradients at $\mu=M_{s}$. For operators with zero engineering dimension, this then translates to a vanishing beta function at $\mu=M_{s}$, suggesting the existence of an unexpected (and ultimately unstable) "UV" fixed point at that location.

In the same vein, it would also be interesting to study the behavior of scattering amplitudes within a full modularinvariant context. We once again expect significant deviations from our field-theoretic expectations at all scales-including those at energies relatively far below the string scale-but it would be interesting to obtain precise information about how this occurs and what shape the deviations take.

Given our results thus far, perhaps the most important and compelling avenue to explore concerns the gauge hierarchy problem. As discussed in the Introduction, it remains our continuing hope that modular symmetries might provide a new perspective on this problem, one that transcends our typical field-theoretic expectations. Some 
ideas in this direction were already sketched in Ref. [19], along with suggestions that the gauge hierarchy problem might be connected with the cosmological-constant problem, and that these both might be closely connected with the question of vacuum stability. It was also advocated in the Conclusions section of Ref. [19] that these insights might be better understood through calculational frameworks that did not involve discarding the contributions of the infinite towers of string states, but which instead incorporated all of these contributions in order to preserve modular invariance and the string finiteness that follows.

The results of this paper enable us to begin the process of fulfilling these ambitions. In particular, the effective potential in Eq. (6.5) is a powerful first step because this result provides a "UV-complete" effective potential which yields the raw expressions for radiative corrections written in terms of the spectrum of whatever theory one may be interested in studying. Moreover it is an expression that is applicable at all energy scales, including the scales associated with the cosmological constant and the electroweak physics where such results are critical.

Given our results, we can develop a string-based reformulation of both of these hierarchy problems. Our expression for the cosmological constant in Eq. (6.2) [or equivalently taking $\lim _{\mu \rightarrow 0} \hat{\Lambda}(\mu)$ ] implicitly furnishes us with a constraint of the form $\operatorname{Str} M^{2} \sim 24 M_{\Lambda}^{4} / \mathcal{M}^{2}$ where $M_{\Lambda} \sim \Lambda^{1 / 4} \approx 2.3 \times 10^{-3} \mathrm{eV}$ is the mass scale associated with the cosmological constant. Likewise, we see that $\Lambda \ll 4 \pi^{2} M_{\mathrm{EW}}^{2} \mathcal{M}^{2}$ where $M_{\mathrm{EW}} \sim \mathcal{O}(100) \mathrm{GeV}$ denotes the electroweak scale. Thus, with $\phi$ representing the Standard Model Higgs and roughly identifying the physical Higgs mass as $\lim _{\mu \rightarrow 0} \hat{m}_{\phi}^{2}(\mu) \sim M_{\mathrm{EW}}^{2}$, we see from Eq. (6.6) that we can obtain a second constraint of the form $\left.\partial_{\phi}^{2} \operatorname{Str} M^{2}\right|_{\phi=0} \sim 24 M_{\mathrm{EW}}^{2} / \mathcal{M}^{2}$. We therefore see that our two hierarchy conditions now respectively take the forms

$$
\left\{\begin{array}{l}
\left.\operatorname{Str} M^{2}\right|_{\phi=0} \sim 24 M_{\Lambda}^{4} / \mathcal{M}^{2}, \\
\left.\partial_{\phi}^{2} \operatorname{Str} M^{2}\right|_{\phi=0} \sim 24 M_{\mathrm{EW}}^{2} / \mathcal{M}^{2}
\end{array}\right.
$$

where we continue to regard our masses $M^{2}$ as functions of the Higgs fluctuations $\phi$, as in Eq. (5.11). To one-loop order, these are the hierarchy conditions that must be satisfied by the spectrum of any modular-invariant string theory. Indeed, substituting the masses in Eq. (5.11), these two conditions reduce to the forms

$$
\left\{\begin{array}{l}
\operatorname{Str} \beta_{0} \sim 24 M_{\Lambda}^{4} / \mathcal{M}^{4}, \\
\operatorname{Str} \beta_{2} \sim 24 M_{\mathrm{EW}}^{2} / \mathcal{M}^{2} .
\end{array}\right.
$$

Although every massive string state has a nonzero $\beta_{0}$ and therefore contributes to the first constraint, only those string states which couple to the Higgs field have a nonzero $\beta_{2}$ and thereby contribute to the second. Of course, given the form of Eq. (5.11), the nonzero $\beta_{i}$ 's for each state are still expected to be $\sim \mathcal{O}(1)$, which is precisely why these constraints are so difficult to satisfy. Moreover, as we know in the case of string models exhibiting charge lattices, these $\beta_{i}$-coefficients are related to the charges of the individual string states and therefore can be discrete in nature.

Given the constraints in Eq. (6.9), it is natural to wonder why there is no hierarchy condition corresponding to $\operatorname{Str} \beta_{1}$. Actually, such a condition exists, although this is not normally treated as a hierarchy constraint. This is nothing but our stability condition $\left.\partial_{\phi} \hat{\Lambda}(\mu, \phi)\right|_{\phi=0}=0$ in Eq. (6.10), which can be considered on the same footing as the other two relations in Eq. (6.3). As we have seen, this leads directly to the relations $\operatorname{Str} \mathbb{Y}=0$ or equivalently $\left.\partial_{\phi} \operatorname{Str} M^{2}\right|_{\phi=0}=0$, which can be considered alongside the relations in Eq. (6.8). This then leads to the constraint $\operatorname{Str} \beta_{1}=0$. Of course, it is always possible that there exists a nonzero Higgs tadpole, as long as this tadpole is sufficiently small as to have remained unobserved (e.g., at colliders, or cosmologically), leading to string models which are not truly stable but only metastable. Such models would be analogous to nonsupersymmetric string models in which the dilaton tadpole is nonvanishing but exponentially suppressed to a sufficient degree that the theory is essentially stable on cosmological timescales [32]. In such cases involving a nonzero Higgs potential, we can define an associated mass scale $M_{\text {stab }}$ which characterizes the maximum possible Higgs instability we can tolerate experimentally and/or observationally. Our corresponding "hierarchy" condition would then take the form

$$
\operatorname{Str} \beta_{1} \lesssim M_{\text {stab }} / \mathcal{M}
$$

Of course, this condition differs from the others in that it does not describe a phenomenological constraint on a particular vacuum but rather helps to determine whether that vacuum even exists. All conditions nevertheless determine whether a given value of $\langle\phi\rangle$ (in this case defined as $\langle\phi\rangle=0$ ) is viable. In general, such "hierarchies" exist for each scalar $\phi$ in the theory.

Despite their fundamentally different natures, these two types of hierarchies can actually be connected to each other. In the case that $\phi$ represents the Standard Model Higgs, this connection will then allow us to relate $M_{\text {stab }}$ to $M_{\mathrm{EW}}$. The fundamental reason for this connection is that a tadpole corresponds to a linear term in an effective potential for the Higgs. This is in addition to the quadratic mass term. However, we can eliminate the linear term by completing the square, which of course simply shifts the corresponding Higgs VEV. The maximum size of this tadpole diagram is therefore also bounded by $M_{\mathrm{EW}}$. More precisely, we find for the Standard Model Higgs that

$$
M_{\mathrm{stab}} \sim 24 M_{\mathrm{EW}}^{3} / \mathcal{M}^{2}
$$


whereupon Eq. (6.10) takes the form

$$
\operatorname{Str} \beta_{1} \lesssim 24 M_{\mathrm{EW}}^{3} / \mathcal{M}^{3} .
$$

Indeed, in this form Eq. (6.12) more closely resembles the relations in Eq. (6.9).

It is remarkable that in string theory the constraints from the cosmological-constant problem and the gauge hierarchy problem in Eq. (6.9) take such similar algebraic forms. Indeed in some sense $\beta_{0}$ and $\beta_{2}$ measure the responses of our individual string states to mass (or gravity) and to fluctuations of the Higgs field, respectively, with $\beta_{2}$ related to the charges of these states with respect to Higgs couplings. It is also noteworthy that these conditions each resemble the so-called "Veltman condition" [35] of field theory. Recall that the Veltman condition for addressing the gauge hierarchy in an effective field theory such as the Standard Model calls for canceling the quadratic divergence of the Higgs mass by requiring the vanishing of the (mass) $)^{2}$ supertrace $\operatorname{Str} M^{2}$ when summed over all light EFT states which couple to the Higgs. However, we now see that in string theory the primary difference is that the supertraces $\operatorname{Str} M^{2}$ in Eq. (6.9) are evaluated over the entire spectrum of string states and not merely the light states within the EFT. This is an important difference because the vanishing of this supertrace when restricted to the EFT generally tells us nothing about its vanishing in the full theory, or vice versa. These are truly independent conditions, and we see that string theory requires the latter, not the former.

One of the virtues of modular invariance-and indeed an indication of its overall power as a robust, unbroken symmetry-is that the string naturalness conditions in Eqs. (6.8) and (6.9) necessarily include the effects of all physics occurring at intermediate scales. This includes, for example, the effects of a possible grand unified theory phase transition. As discussed earlier in this paper, this is true because modular invariance is an exact symmetry governing not only all of the states in the string spectrum but also their interactions. Thus all intermediate-scale physics - even including phase transitions - must preserve modular invariance. This in turn implies that as the masses and degrees of freedom within the theory evolve, they all evolve together in a carefully balanced way such that modular invariance is preserved. Thus, given that relations such as that in Eq. (6.6) are general and rest solely on modular invariance, they too will remain intact. Relations such as those in Eqs. (6.8) and (6.9) then remain valid.

Thus far we have reformulated the constraints associated with the cosmological-constant and gauge hierarchy problems, providing what may be viewed as essentially "stringy" versions of the traditional Veltman condition. However our results also suggest new stringy mechanisms by which such constraints might actually be satisfiedmechanisms by which such hierarchies might actually emerge within a given theory. Given the general running behavior of the Higgs mass in Fig. 3, we observe two interesting features that may be relevant for hierarchy problems. First, let us imagine that we apply our formalism for the running of the Higgs mass in the original unbroken phase of the theory. We will then continue to obtain a result for the Higgs running with the same shape as that shown in Fig. 3, only with the relevant quantities $\Lambda, \mathbb{X}_{1}$, and $\mathbb{X}_{2}$ evaluated in the unbroken phase. Concentrating on the region with $\mu \leq M_{s}$, we see that there is a relatively slow (logarithmic) running which stretches all the way from the string scale $M_{s}$ down to the energy scales associated with the lightest massive string states, followed by a transient "dip" region within which the Higgs mass experiences a sudden local minimum. This therefore provides a natural scenario in which electroweak symmetry breaking might be triggered at an energy scale hierarchically below the fundamental high energy scales in the theory. Note that the dip region indeed produces a minimum for the Higgs mass only if $\operatorname{Str} \mathbb{X}_{2}>0$; otherwise the logarithmic running changes sign and the Higgs mass would already be tachyonic at high energy scales near the string scale, signifying (contrary to assumptions) that our theory was not sitting at a stable minimum in $\phi$-space at high energies. (We also note that even though $\mathbb{X}_{2} \geq 0$, the supertrace $\operatorname{Str} \mathbb{X}_{2}$ can have either sign depending on how these $\mathbb{X}_{2}$ charges are distributed between bosonic and fermionic states.) However, with $\operatorname{Str} \mathbb{X}_{2}>0$, this transient minimum in Fig. 3 will cause the Higgs to become tachyonic as long as

$$
\frac{\pi}{6} \operatorname{Str} \mathbb{X}_{1}+\frac{3}{10} \operatorname{Str} \mathbb{X}_{2} \gtrsim \frac{\xi}{4 \pi^{2}} \frac{\Lambda}{\mathcal{M}^{4}}
$$

where the factor of $3 / 10$ represents the approximate value $\approx 0.3$ parametrizing the "dip depth" from Fig. 3. It is remarkable that this condition links the scale of electroweak symmetry breaking with the value of the one-loop cosmological constant. Just as with our other conditions, this condition can be also expressed as a constraint on the values of our $\beta_{i}$ coefficients:

$$
\frac{9}{5} \operatorname{Str} \beta_{1}^{2}-4 \pi^{2} \operatorname{Str} \beta_{2} \gtrsim \xi \operatorname{Str} \beta_{0} .
$$

This is then our condition for triggering electroweak symmetry breaking at small scales hierarchically below $\mathcal{M}$. Of course, after this breaking occurs, we would need to work in the broken phase wherein $\phi$ returns to representing the Higgs fluctuations relative to the new broken-phase vacuum.

The second feature illustrated within Fig. 3 that may be relevant for the hierarchy problems concerns the scaleduality symmetry $\mu \rightarrow M_{s}^{2} / \mu$. As we have discussed at numerous points throughout this paper, this symmetry implies an equivalence between UV physics and IR physics - an observation which already heralds a major disruption of our understanding of the relationship between 
high and low energy scales compared with field-theoretic expectations. Given that hierarchy problems not only emerge within the context of low-energy EFTs but also assume traditional field-theoretic relationships between UV and IR physics, it is possible to speculate that such hierarchy problems are not fundamental and do not survive in string theory in the manner we normally assume. Furthermore, we have already seen that modular invariance not only leads to this UV/IR mixing but also softens divergences so dramatically that certain otherwisedivergent amplitudes (such as the cosmological constant) are rendered finite. Taken together, these observations suggest that modular invariance may hold the key to an entirely new way of thinking about hierarchy problems-a point originally made in Ref. [19] and which we will develop further in upcoming work [36].

The results of this paper also prompt a number of additional lines of research. For example, although most of our results are completely general and hold across all modular-invariant string theories, much of our analysis in this paper has been restricted to one-loop order. It would therefore be interesting to understand what occurs at higher loops. In this connection, we note that it is often asserted in the string literature that modular invariance is only a oneloop symmetry, seeming to imply that it should no longer apply at higher loops. However, this is incorrect: modular invariance is an exact world sheet symmetry of (perturbative) closed strings, and thus holds at all orders. This symmetry is merely motivated by the need to render oneloop string amplitudes consistent with the underlying conformal invariance of the string world sheet. Once imposed, however, this symmetry affects the entire string model - all masses and interactions, to any order. Likewise, one might wonder whether there are multiloop versions of modular invariance which could also be imposed, similarly motivated by considerations of higher-loop amplitudes. However, it has been shown [37] that within certain closedstring theories, amplitude factorization and physically sensible state projections together ensure that one-loop modular invariance automatically implies multiloop modular invariance. Thus one-loop modular invariance is sufficient, and no additional symmetries of this sort are needed.

Because modular invariance is an exact world sheet symmetry, we expect that certain features we have discussed in this paper (such as the existence of the scaleduality symmetry under $\mu \rightarrow M_{s}^{2} / \mu$ ) will remain valid to all orders. We believe that the same is true of other consequences of modular invariance, such as our supertrace relations and the "misaligned supersymmetry" [17-19] from which they emerge.

That said, modular invariance is a symmetry of closed strings. For this reason, we do not expect modular invariance to hold for type I strings, which contain both closedstring and open-string sectors. However, within type I strings there are tight relations between the closed-string and open-string sectors, and certain remnants of modular invariance survive even into the open-string sectors. For example, certain kinds of misaligned supersymmetry have been found to persist even within open-string sectors [38]. It will therefore be interesting to determine the extent to which the results and techniques of this paper might extend to open strings.

The results described in this paper have clearly covered a lot of territory, stretching from the development of new techniques for calculating Higgs masses to the development of modular-invariant methods of regulating divergences. We have also tackled critical questions concerning UV/IR mixing and the extent to which one can extract effective field theories from modular-invariant string theories, complete with Higgs masses and a cosmological constant that run as functions of a spacetime mass scale. We have demonstrated that there are unexpected relations between the Higgs mass and the one-loop cosmological constant in any modular-invariant string model, and that it is possible to extract an entirely string-based effective potential for the Higgs. Moreover, as indicated in the Introduction, our results apply to all scalars in the theory-even beyond the Standard Model Higgs - and apply whether or not spacetime supersymmetry is present. As such, we anticipate that there exist numerous areas of exploration that may be prompted by these developments. But perhaps most importantly for phenomenological purposes, we believe that the results of this paper can ultimately serve as the launching point for a rigorous investigation of the gauge hierarchy problem in string theory. Much work therefore remains to be done.

\section{ACKNOWLEDGMENTS}

We are happy to thank Carlo Angelantonj, Athanasios Bouganis, and Jens Funke for insightful discussions. The research activities of S.A.A. were supported by STFC Grant No. ST/P001246/1 and partly by a CERN Associateship and Royal-Society/CNRS International Cost Share Award No. IE160590. The research activities of K. R. D. were supported in part by the U.S. Department of Energy under Grant No. DE-FG02-13ER41976/DESC0009913, and also by the U.S. National Science Foundation through its employee IR/D program. The opinions and conclusions expressed herein are those of the authors, and do not represent any funding agencies.

\section{APPENDIX A: EVALUATING THE HIGGS MASS WITH THE MODULAR-INVARIANT REGULATOR: EXPLICIT CALCULATION}

Our goal in Appendices A and B is to provide an explicit calculation of the regulated Higgs mass $\hat{m}_{\phi}^{2}(\rho, a)$ given in Eq. (4.25), and to express the result directly in terms of supertraces over the physical string states. In this appendix we shall focus on the contribution $\left.\hat{m}_{\phi}^{2}(\rho, a)\right|_{\mathcal{X}}$ 
which comes from the terms with nontrivial $\mathcal{X}_{i}$ insertions. The contribution $\left.\hat{m}_{\phi}^{2}(\rho, a)\right|_{\Lambda}$ from the $\Lambda$-term will be discussed in Appendix B.

Because our regulator function $\hat{\mathcal{G}}_{\rho}(a, \tau)$ is built upon the circle partition function $Z_{\text {circ }}(a, \tau)$ defined in Eq. (3.30), the core of our calculation of $\left.\hat{m}_{\phi}^{2}(\rho, a)\right|_{\mathcal{X}}$ ultimately rests on evaluating the integral

$$
P(a) \equiv \int_{\mathcal{F}} \frac{d^{2} \tau}{\tau_{2}^{2}} F(\tau) Z_{\text {circ }}(a, \tau)
$$

where $F(\tau)$ is the modular-invariant string partition function with the $\mathcal{X}_{i}$ insertions indicated in Eq. (4.26). We shall therefore begin by focusing on this integral. Note that the $a \rightarrow 1 / a$ symmetry of $Z_{\text {circ }}(a)$ ensures that $P(a)=P(1 / a)$. Once we have evaluated $P(a)$, we can then easily evaluate the full expression for the $\mathcal{X}_{i}$-dependent contributions to the Higgs mass in Eq. (4.26) via

$$
\left.\hat{m}_{\phi}^{2}(\rho, a)\right|_{\mathcal{X}}=\frac{1}{1+\rho a^{2}} A_{\rho} a^{2} \frac{\partial}{\partial a}[P(\rho a)-P(a)]
$$

where $A_{\rho} \equiv \rho /(\rho-1)$. Given that $P(a)=P(1 / a)$, the expression in Eq. (A2) for the Higgs mass will be invariant under $a \rightarrow 1 /(\rho a)$. We also emphasize that the result of our calculation will be a manifestly finite quantity, as ensured by the presence of the regulator $\hat{\mathcal{G}}_{\rho}(a, \tau)$ in our integrand. Therefore the calculation we shall be performing here is nothing but the direct evaluation of an integral, with no additional regulators needed.

As in Sec. III, our first step is to recast Eq. (A1) as an integral over the strip $\mathcal{S}$ in Eq. (3.3). In order to do this, we first note that we can perform a Poisson resummation of the expression for $Z_{\text {circ }}(a, \tau)$ in Eq. (3.30). Indeed, we can resum either the winding modes or the momentum modes in Eq. (3.30), ultimately obtaining the two alternative expressions

$$
\begin{aligned}
Z_{\text {circ }}(a, \tau) & =a \sum_{j, k} \exp \left(-\frac{\pi a^{2}}{\tau_{2}}|j+k \tau|^{2}\right) \\
& =\frac{1}{a} \sum_{j, k} \exp \left(-\frac{\pi}{a^{2} \tau_{2}}|j+k \tau|^{2}\right)
\end{aligned}
$$

respectively. Indeed, the existence of these two equivalent expressions for $Z_{\text {circ }}$ is nothing but a manifestation of the symmetry of $Z_{\text {circ }}$ under $a \rightarrow 1 / a$. Of course, each of these expressions independently retains the $a \rightarrow 1 / a$ symmetry [since each is equal to $Z_{\text {circ }}(a, \tau)$ ], but this symmetry is no longer manifest.

In principle, we could now proceed using either of the two expressions in Eq. (A3). However, since we shall be most interested in the physics that emerges for $a \ll 1$, we shall find it most useful to continue from the second expression in Eq. (A3). This is the expression in which the momentum modes within $Z_{\text {circ }}$ are Poisson-resummed, as appropriate when the compactification radius $a^{-1}$ is large. Indeed, it is precisely through this resummation that we find $Z_{\text {circ }} \sim a^{-1}$ as $a \rightarrow 0$.

Continuing from Eq. (A3), we next define the greatest common divisor $r \equiv \operatorname{gcd}(j, k)$, where $r=0$ if $j=k=0$, where $r>0$ in all other cases, and where $\operatorname{gcd}(0, k) \equiv|k|$ for all $k$. With this definition, the second line of Eq. (A3) becomes

$$
\begin{aligned}
Z_{\text {circ }}(a, \tau) & =\frac{1}{a}+\frac{1}{a} \sum_{r=1}^{\infty} \sum_{\substack{j, k \\
(j, k)=1}} \exp \left(-\frac{\pi r^{2}}{a^{2} \tau_{2}}|j+k \tau|^{2}\right) \\
& =\frac{1}{a}+\frac{2}{a} \sum_{r=1}^{\infty} \sum_{\substack{j, k \\
(j, k)=1 \\
j+k>0}} \exp \left(-\frac{\pi r^{2}}{a^{2} \tau_{2}}|j+k \tau|^{2}\right) .
\end{aligned}
$$

Note that in these expressions the new $(j, k)$ summations are over values of $j$ and $k$ which are relatively prime. On the first line of Eq. (A7) we have explicitly separated those contributions with $r=0$ from those with $r>0$, while on the second line we have further restricted our sum so that $j+k>0$ [thereby ensuring that if $(j, k)$ is included then $(-j,-k)$ is excluded, and vice versa].

Next, we observe that any modular transformation $\tau \rightarrow \tau^{\prime} \equiv(A \tau+B) /(C \tau+D)$ sends $\tau_{2} \rightarrow \tau_{2}^{\prime} \equiv \tau_{2} /|C \tau+D|^{2}$. We further note that $C$ and $D$ are relatively prime for any such modular transformation (thanks to the constraint $A D-B C=1)$ and that the set of modular transformations consisting of one representative for each possible pair of relatively prime integers $(C, D)$ with $C+D>0$ are precisely those that fill out the coset $\Gamma_{\infty} \backslash \Gamma$. (Indeed, the infinite number of possible choices for $A$ and $B$ in each case generate the distinct cosets.) As a result, when acting on $\mathcal{F}$, these modular transformations fill out the strip $\mathcal{S}$. Thus, multiplying Eq. (A3) by $F(\tau)$, integrating over the fundamental domain $\mathcal{F}$, and then utilizing the unfolding relation in Eq. (3.7) on the second term on the second line of Eq. (A7) yields the result

$$
P(a)=P_{1}(a)+P_{2}(a)
$$

where

$$
\begin{aligned}
P_{1}(a) & \equiv \frac{1}{a} \int_{\mathcal{F}} \frac{d^{2} \tau}{\tau_{2}^{2}} F(\tau) \\
P_{2}(a) & \equiv \frac{2}{a} \int_{\mathcal{S}} \frac{d^{2} \tau}{\tau_{2}^{2}} F(\tau) \sum_{r=1}^{\infty} e^{-\pi r^{2} /\left(a^{2} \tau_{2}\right)} \\
& =\frac{2}{a} \int_{0}^{\infty} \frac{d \tau_{2}}{\tau_{2}^{2}} g\left(\tau_{2}\right) \sum_{r=1}^{\infty} e^{-\pi r^{2} /\left(a^{2} \tau_{2}\right)}
\end{aligned}
$$


Indeed, $P_{1}(a)$ is nothing but our original integral $P(a)$ in the full $a \rightarrow 0$ limit, wherein $Z_{\text {circ }} \rightarrow a^{-1}$.

In principle, our goal at this stage is to evaluate $P_{1}(a)$ and $P_{2}(a)$. Unfortunately, although the sum $P(a) \equiv$ $P_{1}(a)+P_{2}(a)$ leads to a finite result for the Higgs mass in Eq. (A2), the individual terms $P_{1,2}(a)$ do not; instead they lead to expressions which each exhibit the original logarithmic divergence associated with our unregulated Higgs mass. Indeed, these logarithmic divergences only cancel in the Higgs-mass contributions coming from the sum $P(a)$. For this reason, we shall now reshuffle our expressions for $P_{1}(a)$ and $P_{2}(a)$, producing new quantities $P_{1}^{\prime}(a)$ and $P_{2}^{\prime}(a)$ such that each leads to an independently finite contribution to the Higgs mass. To do this, we recall that our Higgs-mass calculation yields $g\left(\tau_{2}\right) \sim c_{0}+c_{1} \tau_{2}$ as $\tau_{2} \rightarrow \infty$, where $c_{0}$ and $c_{1}$ are given in Eq. (4.8). We shall therefore define

$$
\begin{aligned}
& P_{1}^{\prime}(a) \equiv P_{1}(a)-\frac{1}{a} \int_{t}^{\infty} \frac{d \tau_{2}}{\tau_{2}} c_{1}, \\
& P_{2}^{\prime}(a) \equiv P_{2}(a)+\frac{1}{a} \int_{t}^{\infty} \frac{d \tau_{2}}{\tau_{2}} c_{1}
\end{aligned}
$$

where $t$ is an arbitrary finite parameter. As we shall see, the extra terms in Eq. (A7) have the net effect of transferring this logarithmic divergence between the Higgs-mass contributions coming from these separate terms, thereby allowing these divergences to separately cancel. Indeed, for any finite $t$, each of these new quantities $P_{i}^{\prime}(a)$ leads to a finite contribution to the Higgs mass [and $P_{1}^{\prime}(a)$ will even be finite by itself]. Of course, with these extra terms, the new quantities $P_{i}^{\prime}(a)$ are no longer individually modular invariant. However, modular invariance continues to be preserved for their sum, as required. Likewise, although $P_{1}^{\prime}(a)$ and $P_{2}^{\prime}(a)$ will now depend on $t$, all dependence on $t$ will cancel in their sum.

We emphasize that despite a superficial similarity to the nonminimal regulator, the act of passing from $\left\{P_{1}(a), P_{2}(a)\right\}$ to $\left\{P_{1}^{\prime}(a), P_{2}^{\prime}(a)\right\}$ is not one in which we are regulating our Higgs mass by softening or eliminating a net divergence. We are simply performing an algebraic reshuffling of terms, transferring a logarithmic divergence from one contributing expression to another. Indeed, the only regulator in our Higgs-mass calculation remains the $\hat{\mathcal{G}}_{\rho}(a, \tau)$ function with which we started.

Having defined these quantities, we now begin by evaluating $P_{1}^{\prime}(a)$. However, upon comparing Eq. (A7) with Eq. (3.28), we note that

$$
P_{1}^{\prime}(a)=\left.\frac{1}{a} \hat{m}_{\phi}^{2}(t)\right|_{\mathbf{Q}}
$$

where on the right side we are explicitly disregarding the $\Lambda$-term (i.e., keeping only those terms that result from nontrivial $\mathbf{Q}$-insertions). Thus, even though we are not employing the nonminimal regulator in this calculation (and thus we do not interpret $t$ as corresponding to a mass scale), we nevertheless find that $P_{1}^{\prime}(a)$ by itself is algebraically identical to what we would have obtained for the Q-dependent contributions to the Higgs mass using the nonminimal regulator. The same algebraic manipulations that took us from Eq. (3.28) to Eq. (3.29) and ultimately Eq. (4.18) then yield

$$
P_{1}^{\prime}(a)=-\frac{\mathcal{M}^{2}}{2 a}\left[\frac{\pi}{3} \operatorname{Str} \mathbb{X}_{1}+\left(\underset{M=0}{\operatorname{Str}} \mathbb{X}_{2}\right) \log 4 \pi t e^{-\gamma}\right]
$$

We now evaluate $P_{2}^{\prime}(a)$. Given the form of $P_{2}(a)$ in Eq. (A6), we shall begin our evaluation of $P_{2}^{\prime}(a)$ by breaking $P_{2}(a)$ into three contributions: those from massless string states charged under $\mathbb{X}_{1}$; those from massless string states charged under $\mathbb{X}_{2}$; and those from the massive string states charged under $\mathbb{X}_{1}$ and/or $\mathbb{X}_{2}$. Note that only the second of these contributions to $P_{2}(a)$ is divergent. It is therefore within this contribution to $P_{2}^{\prime}(a)$ that we shall absorb the extra divergent term in Eq. (A7). This will allow each of these three contributions to take an explicitly finite form.

We can easily evaluate the first of these contributions:

$$
\begin{aligned}
\left.P_{2}(a)\right|_{\substack{\mathbb{X}_{1} \\
M=0}} & =-\frac{\mathcal{M}^{2}}{a} \int_{0}^{\infty} \frac{d \tau_{2}}{\tau_{2}^{2}} \operatorname{Str}_{M=0} \mathbb{X}_{1} \sum_{r=1}^{\infty} e^{-\pi r^{2} /\left(a^{2} \tau_{2}\right)} \\
& =-\frac{\mathcal{M}^{2}}{a} \underset{M=0}{\operatorname{Str}} \mathbb{X}_{1} \sum_{r=1}^{\infty}\left(\pi r^{2} / a^{2}\right)^{-1} \\
& =-\frac{1}{2} a \mathcal{M}^{2}\left(\frac{\pi}{3} \underset{M=0}{\operatorname{Str} \mathbb{X}_{1}}\right) .
\end{aligned}
$$

By contrast, evaluating the contribution to $P_{2}(a)$ from massless states charged under $\mathbb{X}_{2}$ is more subtle. Including the extra logarithmically divergent term from Eq. (A7), we have

$$
\begin{aligned}
\left.P_{2}^{\prime}(a)\right|_{M=0} ^{\mathrm{x}_{2}}= & -\frac{\mathcal{M}^{2}}{a} \int_{0}^{\infty} \frac{d \tau_{2}}{\tau_{2}} \underset{M=0}{\operatorname{Str}} \mathbb{X}_{2} \sum_{r=1}^{\infty} e^{-\pi r^{2} /\left(a^{2} \tau_{2}\right)} \\
& -\frac{\mathcal{M}^{2}}{2 a} \int_{t}^{\infty} \frac{d \tau_{2}}{\tau_{2}} \underset{M=0}{\operatorname{Str}} \mathbb{X}_{2} .
\end{aligned}
$$

Each line of Eq. (A11) is individually logarithmically divergent, but their sum is not. In order to isolate these divergences algebraically and then cancel them between these two terms, we can insert a factor of $\tau_{2}^{s}$ inside each integral, with $s \leq 0$. We then find explicitly that each term diverges as $s \rightarrow 0$, but that their sum remains finite as $s \rightarrow 0$. Explicitly, we have 


$$
\begin{aligned}
\left.P_{2}^{\prime}(a)\right|_{M=0}= & -\frac{\mathcal{M}^{2}}{a} \int_{0}^{\infty} \frac{d \tau_{2}}{\tau_{2}} \tau_{2}^{s} \operatorname{Str}_{M=0} \mathbb{X}_{2} \sum_{r=1}^{\infty} e^{-\pi r^{2} /\left(a^{2} \tau_{2}\right)} \\
& -\frac{\mathcal{M}^{2}}{2 a} \int_{t}^{\infty} \frac{d \tau_{2}}{\tau_{2}} \tau_{2}^{s} \underset{M=0}{\operatorname{Str}} \mathbb{X}_{2} \\
= & -\frac{\mathcal{M}^{2}}{2 a} \operatorname{Str}_{M=0} \mathbb{X}_{2}\left[2 \pi^{s} a^{-2 s} \Gamma(-s) \zeta(-2 s)-\frac{t^{s}}{s}\right] .
\end{aligned}
$$

Each of the terms inside the square brackets on the final line has an expansion around $s=0$ beginning with a leading simple pole $1 / s$. These then cancel, whereupon the $s \rightarrow 0$ limit leaves behind the net finite contribution

$$
\left.P_{2}^{\prime}(a)\right|_{M=0}=\frac{\mathcal{M}^{2}}{2 a} \operatorname{Str}_{M=0} \mathbb{X}_{2} \log \left(4 \pi a^{2} t e^{-\gamma}\right)
$$

Indeed, although this result continues to depends on $t$, this $t$-dependence ultimately cancels when this result is added to the result coming from Eq. (A9). Thus, as required, none of our results depend on the parameter $t$ that characterized our reshuffling of the logarithmic divergence in Eq. (A7).

Finally, we turn to the contributions to $P_{2}(a)$ from the massive states charged under $\mathbb{X}_{1}$ and/or $\mathbb{X}_{2}$. These are also finite, and are given by

$$
\begin{aligned}
\left.P_{2}(a)\right|_{M>0} & =-\frac{\mathcal{M}^{2}}{a} \sum_{r=1}^{\infty} \int_{0}^{\infty} \frac{d \tau_{2}}{\tau_{2}^{2}}\left[\underset{M>0}{\operatorname{Str}}\left(\mathbb{X}_{1}+\tau_{2} \mathbb{X}_{2}\right) e^{-\pi \alpha^{\prime} M^{2} \tau_{2}-\pi r^{2} /\left(a^{2} \tau_{2}\right)}\right] \\
& =-\frac{2 \mathcal{M}^{2}}{a} \sum_{r=1}^{\infty}\left[\frac{1}{2 \pi} \underset{M>0}{\operatorname{Str}} \mathbb{X}_{1}\left(\frac{a M}{r \mathcal{M}}\right) K_{1}\left(\frac{r M}{a \mathcal{M}}\right)+\underset{M>0}{\operatorname{Str}} \mathbb{X}_{2} K_{0}\left(\frac{r M}{a \mathcal{M}}\right)\right]
\end{aligned}
$$

where $K_{\nu}(z)$ is the modified Bessel function of the second kind.

Thus, combining Eqs. (A9), (A15), (A13), and (A14), we obtain our final result

$$
\begin{aligned}
P(a)= & -\frac{1}{2} \mathcal{M}^{2}\left\{\underset{M=0}{\operatorname{Str}} \mathbb{X}_{1}\left[\frac{\pi}{3}(a+1 / a)\right]+\underset{M=0}{\operatorname{Str}} \mathbb{X}_{2}\left[-\frac{2}{a} \log a\right]\right. \\
& \left.+\underset{M>0}{\operatorname{Str}} \mathbb{X}_{1}\left[\frac{\pi}{3 a}+\frac{2}{\pi} \sum_{r=1}^{\infty}\left(\frac{M}{r \mathcal{M}}\right) K_{1}\left(\frac{r M}{a \mathcal{M}}\right)\right]+\underset{M>0}{\operatorname{Str}} \mathbb{X}_{2}\left[\frac{4}{a} \sum_{r=1}^{\infty} K_{0}\left(\frac{r M}{a \mathcal{M}}\right)\right]\right\} .
\end{aligned}
$$

Indeed, this result is exact for all $a$ and for any modularinvariant theory.

At no step in our calculation for the total $P(a)$ did we break the $a \rightarrow 1 / a$ symmetry. It therefore remains true that $P(a)=P(1 / a)$. However, this symmetry is deeply hidden. Indeed, our manipulations in deriving this result presupposed that $F(\tau)$ in Eq. (A1) is modular invariant, and this in turn provides tight (but not obvious) relative constraints on the supertraces which appear on each line of Eq. (A15). For example, given that modular transformations mix massless and massive string modes, the supertraces over the massless modes in any modular-invariant theory are nontrivially balanced against the supertraces over the massive modesespecially when these supertraces are weighted by extra factors of $M$ and the Bessel functions thereof. We have also seen that $\mathbb{X}_{1}$ and $\mathbb{X}_{2}$ are modular completions of each other, and hence their contributions are also mixed under modular transformations. Thus, although $P(a)$ continues to be invariant under $a \rightarrow 1 / a$, we no longer expect to see this explicitly when our terms are organized in the manner presented in Eq. (A15).

Given this result for $P(a)$, we can now directly calculate $\hat{m}_{\phi}^{2}(\rho, a)$ via Eq. (A2). Our result is

$$
\begin{aligned}
\left.\hat{m}_{\phi}^{2}(\rho, a)\right|_{\mathcal{X}}= & \frac{\mathcal{M}^{2}}{1+\rho a^{2}}\left\{\operatorname{Str}_{M=0} \mathbb{X}_{1}\left[-\frac{\pi}{6}\left(1+\rho a^{2}\right)\right]+\underset{M=0}{\operatorname{Str}} \mathbb{X}_{2}\left[\log a-1-\frac{\log \rho}{\rho-1}\right]\right. \\
& +\operatorname{Str}_{M>0} \mathbb{X}_{1}\left\{-\frac{\pi}{6}-\frac{1}{2 \pi(\rho-1)}\left(\frac{M}{\mathcal{M}}\right)^{2} \times\left[\mathcal{K}_{0}^{(0,1)}\left(\frac{M}{a \mathcal{M}}\right)+\mathcal{K}_{2}^{(0,1)}\left(\frac{M}{a \mathcal{M}}\right)\right]\right\} \\
& \left.+\operatorname{Str}_{M>0} \mathbb{X}_{2}\left\{\frac{2}{\rho-1}\left[\mathcal{K}_{0}^{(0,1)}\left(\frac{M}{a \mathcal{M}}\right)-\frac{1}{\rho} \mathcal{K}_{1}^{(1,2)}\left(\frac{M}{a \mathcal{M}}\right)\right]\right\}\right\}
\end{aligned}
$$

where $\mathcal{K}_{\nu}^{(n, p)}(z)$ are the combinations of Bessel functions in Eq. (4.36). 


\section{APPENDIX B: EVALUATING THE COSMOLOGICAL CONSTANT WITH THE MODULAR-INVARIANT REGULATOR: EXPLICIT CALCULATION}

In Appendix A, we provided an explicit calculation of the first contribution to the total regulated Higgs mass listed in Eq. (4.25). In this appendix, with an eye toward evaluating the second contribution, we now provide an explicit calculation of $\hat{\Lambda}(\rho, a)$.

In any tachyon-free modular-invariant theory, the oneloop cosmological constant $\Lambda$ is finite. As such, evaluating $\Lambda$ requires no regulator. However, we have seen that $\Lambda$ also appears as a contributing term within our total result for the Higgs mass in Eq. (2.55), along with contributions stemming from the nontrivial $\mathcal{X}_{i}$ insertions. The latter contributions are divergent, and thus the Higgs mass requires regularization. However, when we regularize our Higgs mass, for consistency we must apply the same regulator to all of the terms that contribute to the Higgs mass, and this includes the cosmological-constant term as well. Accordingly, in this appendix, we shall evaluate the quantity $\hat{\Lambda}(\rho, a)$ defined in Eq. (4.49) where $\mathcal{Z}$ is the one-loop partition function of our theory with no charge insertions, and express our result in terms of supertraces over only physical string states. Indeed, our result for $\hat{\Lambda}(\rho, a)$ can then be incorporated alongside our result for $\left.\hat{m}_{\phi}^{2}(\rho, a)\right|_{\mathcal{X}}$ from Appendix $\mathrm{A}$ in order to obtain a full expression for the regularized Higgs mass $\hat{m}_{\phi}^{2}(\mu)$.

Our calculation will parallel the calculation presented in Appendix A. In particular, we shall begin by evaluating the core integral

$$
P(a) \equiv \int_{\mathcal{F}} \frac{d^{2} \tau}{\tau_{2}^{2}} \mathcal{Z}(\tau) Z_{\mathrm{circ}}(a, \tau)
$$

which is the same as $P(a)$ in Eq. (A1) except that we have replaced $F(\tau) \rightarrow \mathcal{Z}(\tau)$ within the integrand of Eq. (A1). We therefore now have

$$
g\left(\tau_{2}\right)=-\frac{\mathcal{M}^{4}}{2} \tau_{2}^{-1}\left[\underset{M=0}{\operatorname{Str}} \mathbf{1}+\underset{M>0}{\operatorname{Str}} e^{-\pi \alpha^{\prime} M^{2} \tau_{2}}\right] .
$$

Of course,

$$
\underset{M=0}{\operatorname{Str}} \mathbf{1}=n_{B}-n_{F},
$$

where $n_{B}$ and $n_{F}$ are respectively the numbers of physical massless bosonic and fermionic degrees of freedom in the string spectrum. Proceeding exactly as in Appendix A, we can then separate $P(a)$ into two distinct contributions $P_{1}(a)$ and $P_{2}(a)$ as in Eq. (A3), except with $F(\tau) \rightarrow \mathcal{Z}(\tau)$. However, unlike the situation in Appendix A, there is no need to transfer any divergences between these two terms.
Evaluating $P_{1}(a)$ is straightforward, yielding

$$
P_{1}(a)=\frac{1}{a} \Lambda=\frac{\mathcal{M}^{2}}{24 a} \operatorname{Str} M^{2}
$$

where in passing to the final expression we have followed the derivation in Eq. (4.45). Evaluating $P_{2}(a)$ is also relatively straightforward. The contribution to $P_{2}(a)$ from the massless states-i.e., from the first term in Eq. (B2) - is given by

$$
\begin{aligned}
\left.P_{2}(a)\right|_{M=0} & =-\frac{\mathcal{M}^{4}}{a}\left(n_{B}-n_{F}\right) \sum_{r=1}^{\infty} \int_{0}^{\infty} \frac{d \tau_{2}}{\tau_{2}^{3}} e^{-\pi r^{2} /\left(a^{2} \tau_{2}\right)} \\
& =-\frac{\mathcal{M}^{4}}{a}\left(n_{B}-n_{F}\right) \sum_{r=1}^{\infty} \frac{a^{4}}{\pi^{2} r^{4}} \\
& =-\frac{\mathcal{M}^{4}}{2} \frac{\pi^{2}}{45}\left(n_{B}-n_{F}\right) a^{3} .
\end{aligned}
$$

By contrast, the contribution to $P_{2}(a)$ from the massive states-i.e., from the second term in Eq. (B2) - is given by

$$
\begin{aligned}
\left.P_{2}(a)\right|_{M>0} & =-\frac{\mathcal{M}^{4}}{a} \underset{M>0}{\operatorname{Str}} \sum_{r=1}^{\infty} \int_{0}^{\infty} \frac{d \tau_{2}}{\tau_{2}^{3}} e^{-\pi \tau_{2} \alpha^{\prime} M^{2}-\pi r^{2} /\left(a^{2} \tau_{2}\right)} \\
& =-\frac{\mathcal{M}^{2}}{2} \frac{a}{\pi^{2}} \underset{M>0}{\operatorname{Str}}\left[M^{2} \sum_{r=1}^{\infty} \frac{1}{r^{2}} K_{2}\left(\frac{r M}{a \mathcal{M}}\right)\right]
\end{aligned}
$$

where $K_{2}(z)$ is the order-two modified Bessel function of the second kind. Combining our results from Eqs. (B4), (B5), and (B6) then yields our final expression for $P(a)$ :

$$
\begin{aligned}
P(a)= & \frac{\mathcal{M}^{2}}{24 a} \operatorname{Str} M^{2}-\frac{\mathcal{M}^{4}}{2} \frac{\pi^{2}}{45}\left(n_{B}-n_{F}\right) a^{3} \\
& -\frac{\mathcal{M}^{2}}{2} \frac{a}{\pi^{2}} \underset{M>0}{\operatorname{Str}}\left[M^{2} \sum_{r=1}^{\infty} \frac{1}{r^{2}} K_{2}\left(\frac{r M}{a \mathcal{M}}\right)\right] .
\end{aligned}
$$

Applying the operator in Eq. (A2), we then find that our regulated cosmological constant $\hat{\Lambda}(\rho, a)$ is given by

$$
\begin{aligned}
\hat{\Lambda}(\rho, a)= & \frac{1}{1+\rho a^{2}}\left\{\frac{\mathcal{M}^{2}}{24} \operatorname{Str} M^{2}\right. \\
& -\frac{\pi^{2}}{30} \rho\left(1+\rho+\rho^{2}\right)\left(n_{B}-n_{F}\right)(a \mathcal{M})^{4} \\
& -\frac{1}{4 \pi^{2}} \frac{\rho}{\rho-1} \operatorname{Str}_{M>0} M^{4}\left[\mathcal{K}_{1}^{(-1,0)}\left(\frac{M}{a \mathcal{M}}\right)\right. \\
& +2 \rho \mathcal{K}_{2}^{(-2,-1)}\left(\frac{M}{a \mathcal{M}}\right) \\
& \left.\left.+\mathcal{K}_{3}^{(-1,0)}\left(\frac{M}{a \mathcal{M}}\right)\right]\right\}
\end{aligned}
$$

where $\mathcal{K}_{\nu}^{(n, p)}(z)$ are the Bessel-function combinations defined in Eq. (4.36). 
[1] V. S. Kaplunovsky, Nucl. Phys. B307, 145 (1988); B382, 436(E) (1992).

[2] K. R. Dienes and J. March-Russell, Nucl. Phys. B479, 113 (1996).

[3] K. R. Dienes, A. E. Faraggi, and J. March-Russell, Nucl. Phys. B467, 44 (1996).

[4] E. Kiritsis and C. Kounnas, Nucl. Phys. B442, 472 (1995).

[5] E. Kiritsis, C. Kounnas, P. M. Petropoulos, and J. Rizos, Nucl. Phys. B483, 141 (1997).

[6] E. Kiritsis, C. Kounnas, P. M. Petropoulos, and J. Rizos, Nucl. Phys. B540, 87 (1999).

[7] G. W. Moore, Nucl. Phys. B293, 139 (1987); B299, 847(E) (1988).

[8] K. R. Dienes, Phys. Rev. D 42, 2004 (1990).

[9] K. R. Dienes, Phys. Rev. Lett. 65, 1979 (1990).

[10] R. Rankin, Proc. Cambridge Philos. Soc. 35, 351 (1939).

[11] R. Rankin, Proc. Cambridge Philos. Soc. 35, 357 (1939).

[12] A. Selberg, Arch. Math. Naturvidensk. 43, 47 (1940).

[13] D. Zagier, J. Fac. Sci. Univ. Tokyo 28, 415 (1982).

[14] B. McClain and B. D. B. Roth, Commun. Math. Phys. 111, 539 (1987).

[15] K. O'Brien and C. Tan, Phys. Rev. D 36, 1184 (1987).

[16] D. Kutasov and N. Seiberg, Nucl. Phys. B358, 600 (1991).

[17] K. R. Dienes, Nucl. Phys. B429, 533 (1994).

[18] K. R. Dienes, M. Moshe, and R. C. Myers, Phys. Rev. Lett. 74, 4767 (1995).

[19] K. R. Dienes, Nucl. Phys. B611, 146 (2001).

[20] C. Angelantonj, M. Cardella, S. Elitzur, and E. Rabinovici, J. High Energy Phys. 02 (2011) 024.

[21] C. Angelantonj, I. Florakis, and B. Pioline, Commun. Number Theory Phys. 6, 159 (2012).

[22] C. Angelantonj, I. Florakis, and B. Pioline, J. High Energy Phys. 06 (2012) 070.
[23] C. Angelantonj, I. Florakis, and B. Pioline, J. High Energy Phys. 07 (2013) 181.

[24] B. Pioline, Proc. Symp. Pure Math. 88, 119 (2014).

[25] I. Florakis and B. Pioline, Commun. Number Theory Phys. 11, 337 (2017).

[26] K. R. Dienes and A. E. Faraggi, Nucl. Phys. B457, 409 (1995).

[27] K. R. Dienes, C. F. Kolda, and J. March-Russell, Nucl. Phys. B492, 104 (1997).

[28] R. B. Paris, Math. Eterna 8, 71 (2018), https://www.longdom .org/abstract/the-evaluation-of-single-bessel-function-sums4807.html

[29] K. R. Dienes, E. Dudas, and T. Gherghetta, Phys. Lett. B 436, 55 (1998).

[30] K. R. Dienes, E. Dudas, and T. Gherghetta, Nucl. Phys. B537, 47 (1999).

[31] K. R. Dienes, E. Dudas, and T. Gherghetta, in Proceedings of the 1st European Meeting From the Planck Scale to the Electroweak Scale (Planck 1998), Kazimierz, Poland, 1998 (1998), pp. 613-620, https://cds.cern.ch/record/361067/files/ 9807522.pdf.

[32] S. Abel, K. R. Dienes, and E. Mavroudi, Phys. Rev. D 91, 126014 (2015).

[33] S. R. Coleman and E. J. Weinberg, Phys. Rev. D 7, 1888 (1973).

[34] E. J. Weinberg, arXiv:hep-th/0507214.

[35] M. Veltman, Acta Phys. Pol. B 12, 437 (1981).

[36] S. A. Abel and K. R. Dienes (to be published).

[37] H. Kawai, D. C. Lewellen, J. A. Schwartz, and S. H. H. Tye, Nucl. Phys. B299, 431 (1988).

[38] N. Cribiori, S. Parameswaran, F. Tonioni, and T. Wrase, J. High Energy Phys. 04 (2021) 099. 